\title{
Cubic interaction vertices for continuous-spin fields and arbitrary spin massive fields
}

\author{
R.R. Metsaev \\ Department of Theoretical Physics, P.N. Lebedev Physical Institute, \\ Leninsky prospect 53, Moscow 119991, Russia \\ E-mail: metsaev@lpi.ru
}

ABSTRACT: Light-cone gauge formulation of relativistic dynamics of a continuous-spin field propagating in the flat space is developed. Cubic interaction vertices of continuous-spin massless fields and totally symmetric arbitrary spin massive fields are studied. We consider parity invariant cubic vertices that involve one continuous-spin massless field and two arbitrary spin massive fields and parity invariant cubic vertices that involve two continuousspin massless fields and one arbitrary spin massive field. We construct the complete list of such vertices explicitly. Also we demonstrate that there are no cubic vertices describing consistent interaction of continuous-spin massless fields with arbitrary spin massless fields.

Keywords: Space-Time Symmetries, Field Theories in Higher Dimensions, Higher Spin Symmetry

ArXiv EPrint: 1709.08596 


\section{Contents}

1 Introduction 1

2 Free light-cone gauge continuous-spin fields and arbitrary spin massive and massless fields

3 Restrictions imposed on interaction vertices by Poincaré algebra symmetries and by light-cone gauge dynamical principle

3.1 Equations for parity invariant cubic interaction vertices

4 Parity invariant cubic vertices for one continuous-spin massless field and two massive fields with the same mass values

5 Parity invariant cubic vertices for one continuous-spin massless field and two arbitrary spin massive fields with the different mass values

6 Parity invariant cubic vertices for two continuous-spin massless fields and one arbitrary spin massive field

7 Conclusions

A Notation and conventions

B Continuous-spin field in helicity basis

C Derivation of cubic vertices $p_{[3]}^{-}(4.2),(5.2)$

D Derivation of cubic vertex $p_{[3]}^{-}(6.2)$

E Analysis of equations to vertices for one continuous-spin massless field and two massless fields

F Analysis of equations to vertices for two continuous-spin massless fields and one massless field

\section{Introduction}

In view of certain remarkable features, the theory of continuous-spin gauge field has attracted some interest recently [1-10]. An extensive list of references devoted to various aspects of this topic may be found in refs. [2, 3, 9, 11]. We note interesting discussions about possible interrelations between the string theory and continuous-spin field theory in refs. $[12,13]$. Also, we note that, it turns out that a continuous-spin field can be decomposed into an infinite chain of coupled scalar, vector, and totally symmetric tensor fields which consists of every spin just once. This property of a continuous-spin field triggered our interest in this topic because a similar infinite chain of scalar, vector and totally symmetric fields enters the theory of higher-spin gauge field in AdS space $[14,15]$ and we 
expect therefore some interesting interrelations between continuous-spin gauge field theory and higher-spin gauge theory.

In this paper, we study interacting continuous-spin fields. ${ }^{1}$ Namely, our major aim in this paper is to study interaction of continuous-spin fields with arbitrary spin fields which propagate in flat space. To this end we use a light-cone gauge formulation of relativistic dynamics of fields propagating in flat space. The light-cone formulation discovered in ref. [16] offers conceptual and technical simplifications of approaches to many problems of string theories and modern quantum field theory. Though this formulation hides Lorentz symmetries but eventually turns out to be effective. For example, we mention the lightcone gauge formulation of superstring field theories [17-19]. Recently studied light-cone gauge superspace formulations of some supersymmetric field theories may be found in refs. [20-23]. Various interesting applications of the light-cone formalism to field theories such as QCD are discussed in refs. [24-26].

In this paper, we apply a light-cone formalism for studying vertices describing interaction of continuous-spin fields with arbitrary spin fields. To this end, first, we develop the light-cone gauge $s o(d-2)$ covariant formulation of free continuous-spin fields propagating in the flat space $R^{d-1,1}$ with arbitrary $d \geq 4 .^{2}$ Second, using such formulation and adopting the method for constructing cubic interaction vertices developed in refs. [30-32] for arbitrary spin massive and massless fields, we find cubic vertices describing interaction of continuous-spin fields with arbitrary spin massive fields propagating in $R^{d-1,1}, d \geq 4$. Namely, we find parity invariant cubic vertices describing interaction of one continuous-spin massless field with two arbitrary spin massive fields and parity invariant cubic vertices describing interaction of two continuous-spin massless fields with one arbitrary spin massive field. We provide the complete classification for such vertices. Also, we analyse equations for parity invariant cubic vertices describing interaction of one continuous-spin massless field with two arbitrary spin massless fields and equations for parity invariant cubic vertices describing interaction of two continuous-spin massless fields with one arbitrary spin massless field. We show that such equations do not have solutions. In other words, we demonstrate that, in the framework of $s o(d-2)$ covariant light-cone gauge formalism, there are no parity invariant cubic vertices describing consistent interaction of continuous-spin massless fields with arbitrary spin massless fields.

The long term motivation for our study of parity invariant cubic vertices by using the $s o(d-2)$ covariant light-cone gauge formulation is related to the fact that it is the parity invariant light-cone gauge vertices that can be cast into BRST gauge invariant form in a relatively straightforwardly way. For more discussion of this theme, see Conclusions.

This paper is organized as follows.

In section 2 , we introduce our notation and describe the manifestly $s o(d-2)$ covariant light-cone gauge formulation of free continuous-spin field propagating in $R^{d-1,1}$ space.

\footnotetext{
${ }^{1}$ Continuous-spin field is an infinite component field. Finite component fields with arbitrary but fixed integer values of spin are referred to as arbitrary spin fields in this paper.

${ }^{2}$ In the framework of light-cone gauge helicity formalism, free continuous-spin massless field in $R^{3,1}$ was discussed in ref. [11], while all cubic interaction vertices for arbitrary spin massless fields in $R^{3,1}$ were obtained in refs. [27-29].
} 
Also we recall the well-known light-cone gauge formulation of arbitrary spin massless and massive fields.

In section 3, we study restrictions imposed by the Poincaré algebra symmetries on arbitrary $n$-point interaction vertices. After that we restrict our attention to cubic vertices. We formulate the light-cone gauge dynamical principle and discuss restrictions imposed by this principle on cubic vertices. In other words, we find complete system of equations imposed on cubic vertices by the Poincaré algebra symmetries and the light-cone gauge dynamical principle.

In section 4, we present solution to equations for parity invariant cubic vertices describing interaction of one continuous spin massless field with two arbitrary spin massive fields having the same masses, while, in section 5 , we present solution to equations for parity invariant cubic interaction vertices for one continuous spin massless field and two arbitrary spin massive fields having different masses. We provide the complete classification of the just mentioned cubic interaction vertices.

In section 6 , we present solution to equations for parity invariant cubic vertices describing interaction of two continuous-spin massless fields with one arbitrary spin massive field. Using our solution, we provide the complete classification of such cubic interaction vertices.

In section 7 , we summarize our conclusions and suggest directions for future research.

In appendix A, we describe the basic notation and conventions we use in this paper.

In appendix B, we discuss light-cone gauge formulation of continuous-spin field propagating in $R^{3,1}$ by using realization of physical fields in the helicity basis.

In appendix $\mathrm{C}$, we outline the procedure of derivation of cubic vertices describing interaction of one continuous-spin massless field with two arbitrary spin massive fields, while, in appendix $\mathrm{D}$, we outline the procedure of derivation of cubic vertices for two continuous-spin massless fields and one arbitrary spin massive field.

In appendix E, we discuss equations for cubic vertices describing interaction of one continuous-spin massless fields with two arbitrary spin massless fields, while, in appendix F, we study equations for cubic vertices describing interaction of two continuous-spin massless fields with one arbitrary spin massless field. We demonstrate that such equations do not have consistent solution.

\section{Free light-cone gauge continuous-spin fields and arbitrary spin massive and massless fields}

Poincaré algebra in light-cone frame. Light cone gauge method developed in ref. [16] reduces the problem of finding a new dynamical system to a problem of finding a new (light cone gauge) solution for commutation relations of a basic symmetry algebra. For continuous-spin field and arbitrary spin massive and massless fields that propagate in the flat space $R^{d-1,1}$, basic symmetries are associated with the Poincaré algebra iso $(d-1,1)$. We start therefore with a description of a realization of the Poincaré algebra symmetries on a space of continuous-spin field and arbitrary spin massive and massless fields. In this section, we discuss free light-cone gauge fields. 
The Poincaré algebra $i s o(d-1,1)$ is spanned by the translation generators $P^{\mu}$ and rotation generators $J^{\mu \nu}$ which are generators of the Lorentz algebra $s o(d-1,1)$. The commutation relations of the Poincaré algebra we use are given by ${ }^{3}$

$$
\left[P^{\mu}, J^{\nu \rho}\right]=\eta^{\mu \nu} P^{\rho}-\eta^{\mu \rho} P^{\nu}, \quad\left[J^{\mu \nu}, J^{\rho \sigma}\right]=\eta^{\nu \rho} J^{\mu \sigma}+3 \text { terms }
$$

where $\eta^{\mu \nu}$ stands for the mostly positive flat metric tensor. The translation generators $P^{\mu}$ are considered to be hermitian, while the Lorentz algebra generators $J^{\mu \nu}$ are taken to be anti-hermitian.

In order to discuss the light-cone formulation, we introduce, in place of the Lorentz basis coordinates $x^{\mu}$, the light-cone basis coordinates $x^{ \pm}, x^{i}$ which are defined by the relations

$$
x^{ \pm} \equiv \frac{1}{\sqrt{2}}\left(x^{d-1} \pm x^{0}\right), \quad x^{i}, \quad i=1, \ldots, d-2 .
$$

In what follows, the coordinate $x^{+}$is treated as an evolution parameter. Using notation in $(2.2)$, we note then that the $s o(d-1,1)$ Lorentz algebra vector $X^{\mu}$ is decomposed as $X^{+}, X^{-}, X^{i}$, while scalar product of the $s o(d-1,1)$ Lorentz algebra vectors $X^{\mu}$ and $Y^{\mu}$ is decomposed as

$$
\eta_{\mu \nu} X^{\mu} Y^{\nu}=X^{+} Y^{-}+X^{-} Y^{+}+X^{i} Y^{i} .
$$

From (2.3), we see that in light-cone frame, non vanishing elements of the flat metric are given by $\eta_{+-}=\eta_{-+}=1, \eta_{i j}=\delta_{i j}$, i.e., for the covariant and contravariant components of vectors we have the relations $X^{+}=X_{-}, X^{-}=X_{+}, X^{i}=X_{i}$. In light-cone approach, generators of the Poincaré algebra are separated into the following two groups:

$$
\begin{array}{llll}
P^{+}, & P^{i}, \quad J^{+i}, \quad J^{+-}, & J^{i j}, & \text { kinematical generators; } \\
P^{-}, & J^{-i}, & & \text { dynamical generators. }
\end{array}
$$

For $x^{+}=0$, in the field theoretical realization, kinematical generators (2.4) are quadratic in fields, ${ }^{4}$ while, dynamical generators (2.5) involve quadratic and higher order terms in fields.

In light-cone frame, commutators of the Poincaré algebra generators (2.4), (2.5) are obtained from the ones in (2.1) by using the non vanishing elements of the flat metric, $\eta^{+-}=\eta^{-+}=1, \eta^{i j}=\delta^{i j}$. We assume the following hermitian conjugation rules for the generators of the Poincaré algebra,

$$
P^{ \pm \dagger}=P^{ \pm}, \quad P^{i \dagger}=P^{i}, \quad J^{i j \dagger}=-J^{i j}, \quad J^{+-\dagger}=-J^{+-}, \quad J^{ \pm i \dagger}=-J^{ \pm i} .
$$

In order to provide a field theoretical realization of the Poincaré algebra generators on a space of continuous-spin fields and arbitrary spin massive and massless fields we exploit a light-cone gauge description of the fields. We discuss continuous-spin field and arbitrary spin massive and massless fields in turn.

\footnotetext{
${ }^{3}$ Indices $\mu, \nu, \rho, \sigma=0,1, \ldots, d-1$ are vector indices of the Lorentz algebra $s o(d-1,1)$.

${ }^{4}$ For arbitrary $x^{+} \neq 0$, dynamical generators (2.5) can be presented as $G=G_{1}+x^{+} G_{2}$, where a functional $G_{1}$ is quadratic in fields, while a functional $G_{2}$ involves quadratic and higher order terms in fields.
} 
Continuous-spin massless/massive field. To discuss the light-cone gauge description of a continuous-spin massless/massive field, we introduce the following set of scalar, vector and traceless tensor fields of the $s o(d-2)$ algebra:

$$
\phi^{i_{1} \ldots i_{n}}, \quad n=0,1,2, \ldots, \infty .
$$

In (2.7), fields with $n=0$ and $n=1$ are the respective scalar and vector fields of the $s o(d-2)$ algebra, while fields with $n \geq 2$ are traceless tensor fields of the $s o(d-2)$ algebra,

$$
\phi^{i i i_{3} \ldots i_{n}}=0, \quad n=2,3, \ldots, \infty .
$$

In order to discuss the light-cone gauge formulation of a continuous-spin field in an easy-to-use form we introduce the creation operators $\alpha^{i}, v$ and the respective annihilation operators $\bar{\alpha}^{i}, \bar{v}$,

$$
\left[\bar{\alpha}^{i}, \alpha^{j}\right]=\delta^{i j}, \quad[\bar{v}, v]=1, \quad \bar{v}|0\rangle=0, \quad \bar{\alpha}^{i}|0\rangle=0, \quad \alpha^{i \dagger}=\bar{\alpha}^{i}, \quad v^{\dagger}=\bar{v} .
$$

Throughout this paper, the creation and annihilation operators will be referred to as oscillators. We note that the oscillators $\alpha^{i}, \bar{\alpha}^{i}$ and $v, \bar{v}$ transform in the respective vector and scalar representations of the $s o(d-2)$ algebra. Using the oscillators $\alpha^{i}$, $v$, we collect all fields (2.7) into a ket-vector $|\phi\rangle$ defined as

$$
|\phi(p, \alpha)\rangle=\sum_{n=0}^{\infty} \frac{v^{n}}{n ! \sqrt{n !}} \alpha^{i_{1}} \ldots \alpha^{i_{n}} \phi^{i_{1} \ldots i_{n}}(p)|0\rangle,
$$

where the argument $\alpha$ in (2.10) stands for the oscillators $\alpha^{i}, v$, while the argument $p$ stands for the momenta $p^{i}, \beta$. Ket-vector (2.10) satisfies the following algebraic constraints

$$
\begin{aligned}
\left(N_{\alpha}-N_{v}\right)|\phi\rangle & =0, \quad N_{\alpha}=\alpha^{i} \bar{\alpha}^{i}, \quad N_{v}=v \bar{v}, \\
\bar{\alpha}^{2}|\phi\rangle & =0 .
\end{aligned}
$$

We note that constraint (2.12) amounts to tracelessness constraints (2.8).

Arbitrary spin massive fields. To discuss light-cone gauge description of an arbitrary spin- $s$ massive field, we introduce the following set of scalar, vector, and tensor fields of the $s o(d-2)$ algebra:

$$
\phi^{i_{1} \ldots i_{n}}, \quad n=0,1,2, \ldots, s .
$$

In (2.13), fields with $n=0$ and $n=1$ are the respective scalar and vector fields of the $s o(d-2)$ algebra, while fields with $n \geq 2$ are totally symmetric tensor fields of the $s o(d-2)$ algebra. Physical D.o.F of a massive field in flat space $R^{d-1,1}$ are described by irreps of the $s o(d-1)$ algebra. For the fields $(2.13)$ to be associated with irreps of the $s o(d-1)$ algebra, we should impose a constraint on fields (2.13). To simplify the presentation of the constraint we use the vector oscillators $\alpha^{i}, \bar{\alpha}^{i}(2.9)$ and scalar oscillators $\zeta, \bar{\zeta}$ defined by the relations

$$
[\bar{\zeta}, \zeta]=1, \quad \bar{\zeta}|0\rangle=0, \quad \zeta^{\dagger}=\bar{\zeta}
$$


Using the oscillators $\alpha^{i}, \zeta$, we collect all fields (2.13) into a ket-vector $|\phi\rangle$ defined as

$$
\left|\phi_{s}(p, \alpha)\right\rangle=\sum_{n=0}^{s} \frac{\zeta^{s-n}}{n ! \sqrt{(s-n) !}} \alpha^{i_{1}} \ldots \alpha^{i_{n}} \phi^{i_{1} \ldots i_{n}}(p)|0\rangle,
$$

where the argument $\alpha$ in (2.15) stands for the oscillators $\alpha^{i}, \zeta$, while the argument $p$ stands for the momenta $p^{i}, \beta$. Ket-vector (2.15) satisfies the algebraic constraints

$$
\begin{aligned}
\left(N_{\alpha}+N_{\zeta}-s\right)\left|\phi_{s}\right\rangle & =0, \\
\left(\bar{\alpha}^{2}+\bar{\zeta}^{2}\right)\left|\phi_{s}\right\rangle & =0 .
\end{aligned}
$$

Constraint (2.16) tells us that ket-vector $|\phi\rangle$ (2.15) is a degree- $s$ homogeneous polynomial in the oscillators $\alpha^{i}, \zeta$, while relation (2.17) is the constraint required for the fields (2.13) to be associated with irreps of the $s o(d-1)$ algebra. Sometimes we prefer to use an infinite chain of massive fields which consists of every spin just once. Such chain of massive fields is described by the ket-vector

$$
|\phi(p, \alpha)\rangle=\sum_{s=0}^{\infty}\left|\phi_{s}(p, \alpha)\right\rangle
$$

where, in (2.18), the $\left|\phi_{s}(p, \alpha)\right\rangle$ stands for the ket-vector of spin- $s$ massive field given in $(2.15)$.

Arbitrary spin massless fields. To discuss light-cone gauge description of an arbitrary spin- $s$ massless field, we introduce a rank- $s$ totally symmetric traceless tensor field of the so $(d-2)$ algebra

$$
\phi^{i_{1} \ldots i_{s}}, \quad \phi^{i i i_{3} \ldots i_{s}}=0 .
$$

To simplify the presentation we use oscillators $\alpha^{i}(2.9)$ and introduce the following ketvector:

$$
\left|\phi_{s}(p, \alpha)\right\rangle=\frac{1}{s !} \alpha^{i_{1}} \ldots \alpha^{i_{s}} \phi^{i_{1} \ldots i_{s}}(p)|0\rangle,
$$

where the argument $\alpha$ in (2.20) stands for the oscillators $\alpha^{i}$, while the argument $p$ stands for the momenta $p^{i}, \beta$. Ket-vector (2.20) satisfies the algebraic constraints

$$
\begin{aligned}
\left(N_{\alpha}-s\right)\left|\phi_{s}\right\rangle & =0, \\
\bar{\alpha}^{2}\left|\phi_{s}\right\rangle & =0 .
\end{aligned}
$$

From (2.21), we learn that ket-vector (2.20) is a degree-s homogeneous polynomial in the oscillators $\alpha^{i}$, while the constraint for the ket-vector in (2.22) amounts to the tracelessness constraint for tensor fields in (2.19). Sometimes it is convenient to use an infinite chain of massless fields which consists of every spin just once. Such chain of massless fields is described by the ket-vector

$$
|\phi(p, \alpha)\rangle=\sum_{s=0}^{\infty}\left|\phi_{s}(p, \alpha)\right\rangle
$$

where, in (2.23), the $\left|\phi_{s}(p, \alpha)\right\rangle$ stands for the ket-vector of spin- $s$ massless field given in $(2.20)$. 
Field-theoretical realization of Poincaré algebra. We now discuss a field theoretical realization of the Poincaré algebra on the space of continuous fields and arbitrary spin massive and massless fields. A realization of kinematical generators (2.4) and dynamical generators $(2.5)$ in terms of differential operators acting on the ket-vector $|\phi\rangle$ is given by ${ }^{5}$

\section{Kinematical generators:}

$$
\begin{array}{rlrl}
P^{i} & =p^{i}, & P^{+} & =\beta, \\
J^{+i} & =\partial_{p^{i}} \beta, & J^{+-}=\partial_{\beta} \beta, \\
J^{i j} & =p^{i} \partial_{p^{j}}-p^{j} \partial_{p^{i}}+M^{i j}, &
\end{array}
$$

\section{Dynamical generators:}

$$
\begin{aligned}
P^{-} & =-\frac{p^{i} p^{i}+m^{2}}{2 \beta}, \\
J^{-i} & =-\partial_{\beta} p^{i}+\partial_{p^{i}} P^{-}+\frac{1}{\beta}\left(M^{i j} p^{j}+M^{i}\right),
\end{aligned}
$$

where we use the notation

$$
\beta \equiv p^{+}, \quad \partial_{\beta} \equiv \partial / \partial \beta, \quad \partial_{p^{i}} \equiv \partial / \partial p^{i}
$$

In (2.26), (2.28) and below, the $M^{i j}$ stands for a spin operator of the $s o(d-2)$ algebra,

$$
\left[M^{i j}, M^{k l}\right]=\delta^{j k} M^{i l}+3 \text { terms. }
$$

On spaces of ket-vectors of continuous-spin (2.10), arbitrary spin massive (2.15) and arbitrary spin massless (2.20) fields, the operator $M^{i j}$ is realized as

$$
M^{i j}=\alpha^{i} \bar{\alpha}^{j}-\alpha^{j} \bar{\alpha}^{i}
$$

In (2.27), (2.28), the $m$ is a mass parameter, while the $M^{i}$ is a spin operator. The $m$ and $M^{i}$ satisfy the commutation relations

$$
\begin{aligned}
{\left[M^{i}, M^{j k}\right] } & =\delta^{i j} M^{k}-\delta^{i k} M^{j} \\
{\left[M^{i}, M^{j}\right] } & =-m^{2} M^{i j}
\end{aligned}
$$

We now see that all that remains to complete the description of the differential operators in (2.24)-(2.28) is to provide a realization of the spin operator $M^{i}$ on the ket-vectors of the fields under consideration. On spaces of ket-vectors of continuous-spin (2.10), massive (2.15) and massless (2.20) fields, the operator $M^{i}$ is realized in the following way:

\footnotetext{
${ }^{5}$ In this paper, without loss of generality, the generators of the Poincaré algebra are analysed for $x^{+}=0$.
} 
Continuous-spin field (massless, $m^{2}=0$, and massive, $m^{2}<0$ ):

$$
\begin{array}{rlrl}
M^{i} & =g \bar{\alpha}^{i}+A^{i} \bar{g} & \\
A^{i} & =\alpha^{i}-\alpha^{2} \frac{1}{2 N_{\alpha}+d-2} \bar{\alpha}^{i}, & \bar{g}=-v g_{v}, \\
g & =g_{v} \bar{v}, & & \\
g_{v} & =\left(\frac{1}{\left(N_{v}+1\right)\left(2 N_{v}+d-2\right)} F_{v}\right)^{1 / 2}, & \\
F_{v} & =\kappa^{2}-N_{v}\left(N_{v}+d-3\right) m^{2}, & N_{v}=v \bar{v} . \\
N_{\alpha} & =\alpha^{i} \bar{\alpha}^{i}, &
\end{array}
$$

Arbitrary spin massive field, $m^{2}>0$ :

$$
M^{i}=m\left(\zeta \bar{\alpha}^{i}-\alpha^{i} \bar{\zeta}\right)
$$

Arbitrary spin massless field, $m=0$ :

$$
M^{i}=0
$$

In (2.38), the $m$ stands for mass parameter of continuous-spin field, while $\kappa$ is a dimensionfull real-valued parameter, $\kappa^{2}>0$. We note that $\kappa^{2}$ is realized as eigenvalue of square of Pauli-Lubanski vector operator. Also we note that, for a continuous-spin massless field, we have $m=0$, while for a continuous-spin massive field, we assume $m^{2}<0$.

Realizations of the operator $M^{i}$ for massive field (2.40) and massless field (2.41) are well known from textbook [33]. To our knowledge, the realization of the operator $M^{i}$ for continuous-spin field in $R^{d-1,1}$ with arbitrary $d$ and $m \neq 0$ given in (2.34)-(2.39) has not been discussed in earlier literature. ${ }^{6}$

Having found the realization of the Poincaré algebra generators in terms of differential operators in (2.24)-(2.41) we are ready to provide a field theoretical realization of the Poincaré algebra generators in terms of the ket-vectors $|\phi\rangle$. At the quadratic level, a field theoretical realization of the kinematical generators (2.4) and the dynamical generators (2.5) takes the form

$$
G_{[2]}=\int \beta d^{d-1} p\langle\phi(p)|G| \phi(p)\rangle, \quad d^{d-1} p \equiv d \beta d^{d-2} p,
$$

where $G$ stands for the differential operators given in (2.24)-(2.41), while $G_{[2]}$ stands for the field theoretical generators. The ket-vector $|\phi\rangle$ satisfies the well known Poisson-Dirac commutation relations

$$
\left.\left[|\phi(p, \alpha)\rangle,\left|\phi\left(p^{\prime}, \alpha^{\prime}\right)\right\rangle\right]\right|_{\text {equal } x^{+}}=\frac{\delta^{d-1}\left(p+p^{\prime}\right)}{2 \beta} \Pi,
$$

\footnotetext{
${ }^{6}$ For continuous-spin massless field in $R^{3,1}$ and $R^{4,1}$, the discussion of the spin operator $M^{i}$ can be found in section 2 in ref. [11].
} 
where $\Pi$ stands for the projector on space of the respective ket-vectors of continuous (2.10), massive (2.15) and massless (2.20) fields. Using (2.42), (2.43), we verify the standard commutation relation

$$
\left[|\phi\rangle, G_{[2]}\right]=G|\phi\rangle
$$

The following remarks are in order.

i) In the framework of the Lagrangian approach, the light-cone gauge action is given by

$$
S=\int d x^{+} d^{d-1} p\left\langle\phi(p)\left|\mathrm{i} \beta \partial^{-}\right| \phi(p)\right\rangle+\int d x^{+} P^{-},
$$

where $P^{-}$is the Hamiltonian. Representation for the light-cone gauge action given in $(2.45)$ is valid both for the free and interacting fields. In the theory of free fields, the Hamiltonian is obtained from relations (2.27), (2.42).

ii) The light cone gauge formulation of free continuous-spin field we described in this section can be derived by using the Lorentz covariant and gauge invariant formulation of continuous-spin field in terms of the double-traceless gauge fields we developed in ref. [6] and by applying the standard method for the derivation of light-cone gauge formulation from the Lorentz covariant and gauge invariant formulation. For the case of totally symmetric fields in AdS, the pattern of such derivation can be found in section 3 in ref. [34].

\section{Restrictions imposed on interaction vertices by Poincaré algebra sym- metries and by light-cone gauge dynamical principle}

In theories of interacting fields, the dynamical generators given in (2.5) receive corrections which involve higher powers of fields. Dynamical generators (2.5) can be expanded in fields as

$$
G^{\mathrm{dyn}}=\sum_{n=2}^{\infty} G_{[n]}^{\mathrm{dyn}},
$$

where we use the notation $G_{[n]}^{\text {dyn }}$ in (3.1) to denote a functional that has $n$ powers of ket-vector $|\phi\rangle$. Problem of finding a dynamical system of interacting fields amounts to a problem of finding a non-trivial solution to dynamical generators $G_{[n]}^{\mathrm{dyn}}$ for $n \geq 3$. In this section, we describe restrictions imposed on $G_{[n]}^{\text {dyn }}$ by the Poincaré algebra kinematical and dynamical symmetries. After that we discuss restrictions imposed on $G_{[3]}$ by lightcone gauge dynamical principle. For the reader convenience, we start with a discussion of Poincaré algebra kinematical symmetries of dynamical generators $G_{[n]}^{\mathrm{dyn}}$ for arbitrary $n \geq 3$.

Kinematical symmetries of dynamical generators $G_{[n]}^{\text {dyn }}$ for $n \geq 3$. From the commutators of the dynamical generators (2.5) with the kinematical generators $P^{i}$ and $P^{+}$, we find that the dynamical generators $G_{[n]}^{\mathrm{dyn}}$ with $n \geq 3$ can be cast into the following 
form:

$$
\begin{aligned}
& P_{[n]}^{-}=\int d \Gamma_{n}\left\langle\Phi_{[n]}|| p_{[n]}^{-}\right\rangle, \\
& J_{[n]}^{-i}=\int d \Gamma_{n}\left\langle\Phi_{[n]} \mid j_{[n]}^{-i}\right\rangle+\left(\mathbf{X}_{[n]}^{i}\left\langle\Phi_{[n]}\right|\right)\left|p_{[n]}^{-}\right\rangle,
\end{aligned}
$$

where we use the notation

$$
\begin{aligned}
\left\langle\Phi_{[n]}\right| & \equiv \prod_{a=1}^{n}\left\langle\phi\left(p_{a}, \alpha_{a}\right)\right|, \quad\left\langle\phi\left(p_{a}, \alpha_{a}\right)|\equiv| \phi\left(p_{a}, \alpha_{a}\right)\right\rangle^{\dagger} \\
d \Gamma_{n} & \equiv(2 \pi)^{d-1} \delta^{d-1}\left(\sum_{a=1}^{n} p_{a}\right) \prod_{a=1}^{n} \frac{d^{d-1} p_{a}}{(2 \pi)^{(d-1) / 2}} \\
\mathbf{X}_{[n]}^{i} & \equiv-\frac{1}{n} \sum_{a=1}^{n} \partial_{p_{a}^{i}} .
\end{aligned}
$$

The $n$-point densities $\left|p_{[n]}^{-}\right\rangle$and $\left|j_{[n]}^{-i}\right\rangle$ entering the respective generators $P_{[n]}^{-}$and $J_{[n]}^{-i}(3.2)$, (3.3) can be presented as

$$
\begin{aligned}
\left|p_{[n]}^{-}\right\rangle & =p_{[n]}^{-}\left(p_{a}, \beta_{a}, \alpha_{a}\right)|0\rangle, \\
\left|j_{[n]}^{-i}\right\rangle & =j_{[n]}^{-i}\left(p_{a}, \beta_{a}, \alpha_{a}\right)|0\rangle .
\end{aligned}
$$

In this section and below, we use the indices $a, b=1, \ldots, n$ to label fields entering $n$-point interaction vertex. The Dirac $\delta$ - functions in (3.5) respect the conservation laws for the momenta $p_{a}^{i}$ and $\beta_{a}$. We note that argument $p_{a}$ in (3.4), (3.5) stands for the momenta $p_{a}^{i}$, $\beta_{a}$. As seen from (3.7), (3.8), the densities $p_{[n]}^{-}$and $j_{[n]}^{-i}$ depend on the momenta $p_{a}^{i}, \beta_{a}$, and the quantity $\alpha_{a}$ which is shortcut for spin variables. Namely, for continuous-spin field, the shortcut $\alpha_{a}$ stands for the set of oscillators $\alpha_{a}^{i}, v_{a}$, while for massive and massless fields, the shortcut $\alpha_{a}$ stands for the respective sets of oscillators $\alpha_{a}^{i}, \zeta_{a}$ and $\alpha_{a}^{i}$. In this paper, the density $p_{[n]}^{-}$will often be referred to as an $n$-point interaction vertex. For $n=3$, the density $p_{[n]}^{-}$will be referred to as cubic interaction vertex.

$\boldsymbol{J}^{+-}$-symmetry. Commutators of the dynamical generators $P^{-}, J^{-i}$ with the kinematical generator $J^{+-}$lead to the following equations for the densities:

$$
\begin{aligned}
& \sum_{a=1}^{n} \beta_{a} \partial_{\beta_{a}}\left|p_{[n]}^{-}\right\rangle=0, \\
& \sum_{a=1}^{n} \beta_{a} \partial_{\beta_{a}}\left|j_{[n]}^{-i}\right\rangle=0 .
\end{aligned}
$$

$\boldsymbol{J}^{i j}$-symmetries. Commutators of the dynamical generators $P^{-}, J^{-i}$ with the kinematical generators $J^{i j}$ lead to the following equations for the densities:

$$
\begin{aligned}
& \sum_{a=1}^{n}\left(p_{a}^{i} \partial_{p_{a}^{j}}-p_{a}^{j} \partial_{p_{a}^{i}}+M_{a}^{i j}\right)\left|p_{[n]}^{-}\right\rangle=0 \\
& \sum_{a=1}^{n}\left(p_{a}^{i} \partial_{p_{a}^{j}}-p_{a}^{j} \partial_{p_{a}^{i}}+M_{a}^{i j}\right)\left|j_{[n]}^{-k}\right\rangle=\delta^{j k}\left|j_{[n]}^{-i}\right\rangle-\delta^{i k}\left|j_{[n]}^{-j}\right\rangle .
\end{aligned}
$$


$\boldsymbol{J}^{+i}$-symmetries. From the commutators of the dynamical generators $P^{-}, J^{-i}$ with the kinematical generators $J^{+i}$, we learn that the densities $p_{[n]}^{-}$and $j_{[n]}^{-i}$ depend on the momenta $p_{a}^{i}$ through the new momentum variables $\mathbb{P}_{a b}^{i}$ defined by the relation

$$
\mathbb{P}_{a b}^{i} \equiv p_{a}^{i} \beta_{b}-p_{b}^{i} \beta_{a}
$$

Thus we see that the densities $p_{[n]}^{-}$and $j_{[n]}^{-i}$ turn out to be functions of $\mathbb{P}_{a b}^{i}$ in place of $p_{a}^{i}$,

$$
p_{[n]}^{-}=p_{[n]}^{-}\left(\mathbb{P}_{a b}, \beta_{a}, \alpha_{a}\right), \quad j_{[n]}^{-i}=j_{[n]}^{-i}\left(\mathbb{P}_{a b}, \beta_{a}, \alpha_{a}\right) .
$$

To summarize our study of restrictions imposed by the kinematical symmetries we note that the commutators between the dynamical generators $P^{-}, J^{-i}$ and the kinematical generators $J^{+-}, J^{i j}$ amount to equations given in (3.9)-(3.12), while, from the commutators between the dynamical generators $P^{-}, J^{-i}$ and the kinematical generators $J^{+i}$, we learn that the $n$-point densities $p_{[n]}^{-}, j_{[n]}^{-i}$ turn out to be functions of the new momenta $\mathbb{P}_{a b}^{i}$ in place of the generic momenta $p_{a}^{i}$.

Using definition of the new momenta $\mathbb{P}_{a b}^{i}$ (3.13) and the conservation laws for the momenta $p_{a}^{i}, \beta_{a}$, we verify that there are only $n-2$ independent new momenta $\mathbb{P}_{a b}^{i}$. For example, for $n=3$, there is only one independent $\mathbb{P}_{a b}^{i}$ (see relations (3.16) below). This simplifies study of restrictions imposed by kinematical symmetries on dynamical generators. To demonstrate this we consider kinematical symmetries for cubic densities $p_{[3]}^{-}$and $j_{[3]}^{-i}$.

Kinematical symmetries of cubic densities. Taking into account the momentum conservation laws

$$
p_{1}^{i}+p_{2}^{i}+p_{3}^{i}=0, \quad \beta_{1}+\beta_{2}+\beta_{3}=0,
$$

it is easy to check that the momenta $\mathbb{P}_{12}^{i}, \mathbb{P}_{23}^{i}, \mathbb{P}_{31}^{i}$ are expressed in terms of a new momentum $\mathbb{P}^{i}$,

$$
\mathbb{P}_{12}^{i}=\mathbb{P}_{23}^{i}=\mathbb{P}_{31}^{i}=\mathbb{P}^{i},
$$

where a new momentum $\mathbb{P}^{i}$ is defined by the following relations:

$$
\mathbb{P}^{i} \equiv \frac{1}{3} \sum_{a=1,2,3} \check{\beta}_{a} p_{a}^{i}, \quad \check{\beta}_{a} \equiv \beta_{a+1}-\beta_{a+2}, \quad \beta_{a} \equiv \beta_{a+3}
$$

The use of the momentum $\mathbb{P}^{i}(3.17)$ is preferable because this momentum is manifestly invariant under cyclic permutations of the external line indices $1,2,3$. Thus the cubic densities $p_{[3]}^{-}$and $j_{[3]}^{-i}$ are eventually a functions of the momenta $\mathbb{P}^{i}, \beta_{a}$ and the spin variables $\alpha_{a}$ :

$$
p_{[3]}^{-}=p_{[3]}^{-}\left(\mathbb{P}, \beta_{a}, \alpha_{a}\right), \quad j_{[3]}^{-i}=j_{[3]}^{-i}\left(\mathbb{P}, \beta_{a}, \alpha_{a}\right) .
$$

The fact that momenta $p_{a}^{i}$ enter cubic densities though the momentum $\mathbb{P}^{i}$ allows us to simplify the kinematical symmetry equations given in (3.9)-(3.12). Namely, it is easy to check that, in terms of densities (3.18), the kinematical symmetry equations (3.9)-(3.12) can be represented as follows. 
$J^{+-}$-symmetry equations:

$$
\begin{aligned}
\mathbf{J}^{+-}\left|p_{[3]}^{-}\right\rangle & =0, \\
\mathbf{J}^{+-}\left|j_{[3]}^{-i}\right\rangle & =0, \\
\mathbf{J}^{+-} & \equiv \mathbb{P}^{j} \partial_{\mathbb{P}^{j}}+\sum_{a=1,2,3} \beta_{a} \partial_{\beta_{a}} .
\end{aligned}
$$

\section{$J^{i j}$-symmetry equations:}

$$
\begin{aligned}
\mathbf{J}^{i j}\left|p_{[3]}^{-}\right\rangle & =0, \\
\mathbf{J}^{i j}\left|j_{[3]}^{-k}\right\rangle & =\delta^{j k}\left|j_{[3]}^{-i}\right\rangle-\delta^{i k}\left|j_{[3]}^{-j}\right\rangle, \\
\mathbf{J}^{i j} & \equiv \mathbf{L}^{i j}(\mathbb{P})+\mathbf{M}^{i j}, \quad \mathbf{L}^{i j}(\mathbb{P}) \equiv \mathbb{P}^{i} \partial_{\mathbb{P}^{j}}-\mathbb{P}^{j} \partial_{\mathbb{P}^{i}}, \quad \mathbf{M}^{i j} \equiv \sum_{a=1,2,3} M_{a}^{i j} .
\end{aligned}
$$

Obviously, the kinematical symmetries do not admit to fix vertices uniquely. Therefore we proceed with discussion of restrictions imposed by dynamical symmetries.

Dynamical symmetries of cubic densities. In this paper, restrictions on the interaction vertices imposed by commutation relations between the dynamical generators will be referred to as dynamical symmetry restrictions. We now discuss restrictions imposed on cubic interaction vertices by the dynamical symmetries of the Poincare algebra. In other words, we consider the commutators

$$
\left[P^{-}, J^{-i}\right]=0, \quad\left[J^{-i}, J^{-j}\right]=0
$$

In the cubic approximation, commutators (3.25) amount to the following commutators:

$$
\begin{aligned}
{\left[P_{[2]}^{-}, J_{[3]}^{-i}\right]+\left[P_{[3]}^{-}, J_{[2]}^{-i}\right] } & =0, \\
{\left[J_{[2]}^{-i}, J_{[3]}^{-j}\right]+\left[J_{[3]}^{-i}, J_{[2]}^{-j}\right] } & =0 .
\end{aligned}
$$

From commutators (3.26), we obtain the following equation for the cubic densities $\left|p_{[3]}^{-}\left(\mathbb{P}, \beta_{a}, \alpha_{a}\right)\right\rangle$ and $\left|j_{[3]}^{-i}\left(\mathbb{P}, \beta_{a}, \alpha_{a}\right)\right\rangle$,

$$
\mathbf{P}^{-}\left|j_{[3]}^{-i}\right\rangle=-\mathbf{J}^{-i \dagger}\left|p_{[3]}^{-}\right\rangle,
$$

where we use the notation

$$
\begin{aligned}
\mathbf{P}^{-} & \equiv \sum_{a=1,2,3} P_{a}^{-}, & P_{a}^{-} & \equiv-\frac{p_{a}^{i} p_{a}^{i}+m_{a}^{2}}{2 \beta_{a}} \\
\mathbf{J}^{-i \dagger} & \equiv \sum_{a=1,2,3} J_{a}^{-i \dagger}, & J_{a}^{-i \dagger} & \equiv p_{a}^{i} \partial_{\beta_{a}}-p_{a}^{-} \partial_{p_{a}^{i}}-\frac{1}{\beta_{a}} M_{a}^{i j} p_{a}^{j}+\frac{1}{\beta_{a}} M_{a}^{i \dagger} .
\end{aligned}
$$


Quantities $\mathbf{P}^{-}$and $\mathbf{J}^{-i \dagger}$ defined in (3.29), (3.30) can be expressed in terms of the momentum $\mathbb{P}^{i}$ (see appendix $\mathrm{A}$ in ref. [31]):

$$
\begin{aligned}
\mathbf{P}^{-} & =\frac{\mathbb{P}^{i} \mathbb{P}^{i}}{2 \beta}-\sum_{a=1,2,3} \frac{m_{a}^{2}}{2 \beta_{a}}, \\
\mathbf{J}^{-i \dagger} & =-\frac{\mathbb{P}^{i}}{\beta} \mathbb{N}_{\beta}+\frac{1}{\beta} \mathbb{M}^{i j} \mathbb{P}^{j}+\sum_{a=1,2,3} \frac{\check{\beta}_{a}}{6 \beta_{a}} m_{a}^{2} \partial_{\mathbb{P}^{i}}+\frac{1}{\beta_{a}} M_{a}^{i \dagger}, \\
\beta & \equiv \beta_{1} \beta_{2} \beta_{3}, \\
\mathbb{N}_{\beta} & \equiv \frac{1}{3} \sum_{a=1,2,3} \check{\beta}_{a} \beta_{a} \partial_{\beta_{a}}, \quad \mathbb{M}^{i j} \equiv \frac{1}{3} \sum_{a=1,2,3} \check{\beta}_{a} M_{a}^{i j}, \quad \check{\beta}_{a} \equiv \beta_{a+1}-\beta_{a+2} .
\end{aligned}
$$

Equation (3.28) allows us to express the density $\left|j_{[3]}^{-i}\right\rangle$ in terms of the vertex $\left|p_{[3]}^{-}\right\rangle$,

$$
\left|j_{[3]}^{-i}\right\rangle=-\left(\mathbf{P}^{-}\right)^{-1} \mathbf{J}^{-i \dagger}\left|p_{[3]}^{-}\right\rangle .
$$

Plugging $\left|j_{[3]}^{-i}\right\rangle$ (3.35) into (3.27), we check that commutators (3.27) are fulfilled. Plugging $\left|j_{[3]}^{-i}\right\rangle$ (3.35) into kinematical symmetries equations (3.20), (3.23) we verify that, if the vertex $\left|p_{[3]}^{-}\right\rangle$satisfies eqs. (3.19), (3.22) then eqs. (3.20), (3.23) are also fulfilled. Thus, in the cubic approximation, we checked that eqs. (3.19), (3.22), (3.35) provide the complete list of equations obtained from all commutation relations of the Poincaré algebra.

Light-cone gauge dynamical principle. Equations (3.19), (3.22), (3.35) do not admit to fix the vertex $\left|p_{[3]}^{-}\right\rangle$uniquely. In order to fix the vertex $\left|p_{[3]}^{-}\right\rangle$uniquely we impose additional restrictions on the vertex $\left|p_{[3]}^{-}\right\rangle$. These additional restrictions are referred to as light-cone gauge dynamical principle in this paper and they are formulated as follows.

i) The densities $\left|p_{[3]}^{-}\right\rangle,\left|j_{[3]}^{-i}\right\rangle$ should be expandable in the momentum $\mathbb{P}^{i} ;^{7}$

ii) The density $\left|p_{[3]}^{-}\right\rangle$should satisfy the restriction

$$
\left|p_{[3]}^{-}\right\rangle \neq \mathbf{P}^{-}|V\rangle, \quad|V\rangle \text { is expandable in } \mathbb{P}^{i},
$$

where $\mathbf{P}^{-}$is given in (3.31).

iii) The densities $\left|p_{[3]}^{-}\right\rangle,\left|j_{[3]}^{-i}\right\rangle$, and density $|V\rangle$ (3.36) should not involve $\left(\mathbf{P}^{-}\right)^{\gamma}$-terms, $\gamma<0$.

We note that requirement (3.36) is related to field redefinitions. Ignoring requirement (3.36) leads to vertices which can be removed by field redefinitions. As we are interested in the vertices that cannot be removed by using field redefinitions, we impose the requirement in (3.36). Also we note that the assumptions i) and iii) are the light-cone counterpart of locality condition commonly used in Lorentz covariant formulations.

\footnotetext{
${ }^{7}$ If a function $f(x)$ is expandable in power series in $x$, then we refer to such function as function expandable in $x$.
} 
Complete system of equations for cubic vertex. To summarize the discussion in this section, we note that, for the cubic vertex given by

$$
\left|p_{[3]}^{-}\right\rangle=p_{[3]}^{-}\left(\mathbb{P}, \alpha_{a}, \beta_{a}\right)|0\rangle,
$$

the complete system of equations which remains to be solved takes the form

$$
\begin{array}{lr}
\mathbf{J}^{+-}\left|p_{[3]}^{-}\right\rangle=0, & \text { kinematical } J^{+-}-\text {symmetry; } \\
\mathbf{J}^{i j}\left|p_{[3]}^{-}\right\rangle=0, & \text { kinematical } J^{i j}-\text { symmetries; } \\
\left|j_{[3]}^{-i}\right\rangle=-\left(\mathbf{P}^{-}\right)^{-1} \mathbf{J}^{-i \dagger}\left|p_{[3]}^{-}\right\rangle, & \text {dynamical } P^{-}, J^{-i} \text { symmetries } ; \\
& \text { Light-cone gauge dynamical principle: } \\
\left|p_{[3]}^{-}\right\rangle \text {and }\left|j_{[3]}^{-i}\right\rangle & \text { are expandable in } \mathbb{P}^{i} ; \\
\left|p_{[3]}^{-}\right\rangle \neq \mathbf{P}^{-}|V\rangle, \quad|V\rangle \text { is expandable in } \mathbb{P}^{i} ; \\
\left|p_{[3]}^{-}\right\rangle,\left|j_{[3]}^{-i}\right\rangle,|V\rangle \quad \text { do not involve }\left(\mathbf{P}^{-}\right)^{\gamma} \text {-terms, } \quad \gamma<0 .
\end{array}
$$

Eqs. (3.38)-(3.43) constitute the complete system of equations which admit to fix the cubic vertex $p_{[3]}^{-}$uniquely. Operators $\mathbf{J}^{+-}, \mathbf{J}^{i j}, \mathbf{P}^{-}, \mathbf{J}^{-i \dagger}$ entering equations (3.38)-(3.43) are defined in (3.21), (3.24), (3.31), (3.32) respectively. Let us remark that, if we consider the Yang-Mills and Einstein theories, then it can be verified that eqs. (3.38)-(3.40) and the light-cone gauge dynamical principle (3.41)-(3.43) admit to fix the cubic interaction vertices unambiguously (up to coupling constants). It seems then reasonable to use eqs. (3.38)(3.40) and the light-cone gauge dynamical principle (3.41)-(3.43) for studying the cubic interaction vertices of the continuous-spin field theory.

\subsection{Equations for parity invariant cubic interaction vertices}

We recall that we study parity invariant cubic vertices for one continuous-spin massless field and two arbitrary spin massive fields and parity invariant cubic vertices for two continuous-spin massless fields and one arbitrary spin massive field. Namely, using the shortcut $(0, \kappa)_{\mathrm{CSF}}$ for continuous-spin massless field and the shortcut $(m, s)$ for arbitrary but fixed spin- $s$ massive field with mass parameter $m$, we are going to consider cubic vertices for the following fields:

$$
\begin{aligned}
& \left(m_{1}, s_{1}\right)-\left(m_{2}, s_{2}\right)-\left(0, \kappa_{3}\right)_{\mathrm{CSF}} \quad \text { two massive fields and one continuous-spin massless field } \\
& \left(0, \kappa_{1}\right)_{\mathrm{CSF}^{-}}\left(0, \kappa_{2}\right)_{\mathrm{CSF}^{-}}\left(m_{3}, s_{3}\right)
\end{aligned}
$$

Our notation in (3.44) implies that the mass- $m_{1}$, spin- $s_{1}$ and mass- $m_{2}$, spin- $s_{2}$ massive fields carry external line indices $a=1,2$, while the continuous-spin massless field corresponds to $a=3$. From our notation in (3.45), we learn that continuous-spin massless fields carry external line indices $a=1,2$, while the mass- $m_{3}$, spin- $s_{3}$ massive field corresponds to $a=3$.

In general, besides the momentum variables $\mathbb{P}^{i}, \beta_{1}, \beta_{2}, \beta_{3}$, vertex (3.37) depends on oscillators that involved in the ket-vectors entering $P_{[3]}^{-}(3.4)$. Taking this into account 
and recalling the definition of the ket-vectors for continuous-spin field (2.10) and massive field (2.15), we note that cubic vertices describing interactions of the fields in (3.44) and (3.45) depend on the following respective set of the oscillators and the momenta:

$$
\begin{array}{llll}
\mathbb{P}^{i}, & \alpha_{1}^{i}, \zeta_{1}, \beta_{1}, & \alpha_{2}^{i}, \zeta_{2}, \beta_{2}, & \alpha_{3}^{i}, v_{3}, \beta_{3} ; \\
\mathbb{P}^{i}, & \alpha_{1}^{i}, v_{1}, \beta_{1}, & \alpha_{2}^{i}, v_{2}, \beta_{2}, & \alpha_{3}^{i}, \zeta_{3}, \beta_{3} .
\end{array}
$$

Taking into account variables in (3.46), (3.47), we now analyze restrictions (3.38)-(3.43) in turn.

i) First, we analyze the restrictions imposed by the $J^{i j}$-symmetries (3.39) which tell us that the vertex $p_{[3]}^{-}(3.37)$ depend on invariants of the $s o(d-2)$ algebra. The scalar oscillators $\zeta_{a}, v_{a}$ and momenta $\beta_{a}$ are invariants of the $s o(d-2)$ algebra. The remaining invariants can be built by using the momentum $\mathbb{P}^{i}$, the vector oscillators $\alpha_{a}^{i}$, the delta-Kroneker $\delta^{i j}$, and the Levi-Civita symbol $\epsilon^{i_{1} \ldots i_{d-2}}$. Vertices that do not involve the antisymmetric Levi-Civita symbol are referred to as parity invariant vertices, while vertices involving one antisymmetric Levi-Civita symbol are referred to as parity non-invariant vertices. In this paper, we focus on the parity invariant vertices. This implies that invariants of the $s o(d-2)$ algebra that can be built by using $\mathbb{P}^{i}, \alpha_{a}^{i}$, and $\delta^{i j}$ are given by

$$
\mathbb{P}^{i} \mathbb{P}^{i}, \quad \alpha_{a}^{i} \mathbb{P}^{i}, \quad \alpha_{a}^{i} \alpha_{b}^{i} .
$$

Note that, if $P_{[3]}^{-}(3.2)$ involves the bra-vector of continuous-spin field $\left\langle\phi\left(p_{a}, \alpha_{a}\right)\right|$, then in view of constraint (2.12), the invariant $\alpha_{a}^{i} \alpha_{a}^{i}$ does not contribute to the $P_{[3]}^{-}$, while, if $P_{[3]}^{-}(3.2)$ involves bra-vector of massive field $\left\langle\phi\left(p_{a}, \alpha_{a}\right)\right|$, then in view of constraint (2.17), the contribution of invariant $\alpha_{a}^{i} \alpha_{a}^{i}$, can be replaced by the $\left(-\zeta_{a}^{2}\right)$.

To summarize the discussion of $J^{i j}$-symmetries, we note that general solution to parity invariant cubic vertices for the fields (3.44) and (3.45) that respect $J^{i j}$-symmetries and lead to the nontrivial $P_{[3]}^{-}$is given by the following respective expressions

$$
\begin{aligned}
& p_{[3]}^{-}=p_{[3]}^{-}\left(\mathbb{P}^{i} \mathbb{P}^{i}, \beta_{a}, \alpha_{a}^{i} \mathbb{P}^{i}, \alpha_{a a+1}, \zeta_{1}, \zeta_{2}, v_{3}\right), \\
& p_{[3]}^{-}=p_{[3]}^{-}\left(\mathbb{P}^{i} \mathbb{P}^{i}, \beta_{a}, \alpha_{a}^{i} \mathbb{P}^{i}, \alpha_{a a+1}, v_{1}, v_{2}, \zeta_{3}\right) .
\end{aligned}
$$

In (3.49), (3.50) and below, the shortcut $p_{[3]}^{-}\left(q_{a}\right)$ implies that $p_{[3]}^{-}$depends on $q_{1}, q_{2}, q_{3}$.

ii) We now analyse the restriction in (3.42), (3.43). One can demonstrate that, using field redefinitions, we can remove terms in (3.49), (3.50) which are proportional to $\mathbb{P}^{i} \mathbb{P}^{i}$ (see appendix B in ref. [31]). In other words, we can drop down the dependence on $\mathbb{P}^{i} \mathbb{P}^{i}$ in the vertices $p_{[3]}^{-}(3.49),(3.50)$. Representation for the vertices in which they do not depend on $\mathbb{P}^{i} \mathbb{P}^{i}$ will be referred to minimal scheme in this paper. Obviously, in the minimal scheme, vertices satisfy eqs. (3.42), (3.43) automatically. Thus, in the minimal scheme, vertices (3.49), (3.50) take the form

$$
\begin{aligned}
& p_{[3]}^{-}=p_{[3]}^{-}\left(\beta_{a}, B_{a}, \alpha_{a a+1}, \zeta_{1}, \zeta_{2}, v_{3}\right), \\
& p_{[3]}^{-}=p_{[3]}^{-}\left(\beta_{a}, B_{a}, \alpha_{a a+1}, v_{1}, v_{2}, \zeta_{3}\right),
\end{aligned}
$$


where in (3.51), (3.52) and below we use the notation

$$
B_{a} \equiv \frac{\alpha_{a}^{i} \mathbb{P}^{i}}{\beta_{a}}, \quad \alpha_{a b} \equiv \alpha_{a}^{i} \alpha_{b}^{i}
$$

Note also that, in place of the variables $\alpha_{a}^{i} \mathbb{P}^{i}$ appearing in (3.49), (3.50), we use rescaled variables $B_{a}$ (3.53) in expressions (3.51), (3.52). We now see that dependence of vertices $(3.51),(3.52)$ on the momentum $\mathbb{P}^{i}$ enters through the variable $B_{a}(3.53)$.

iii) We now proceed with vertices in (3.51), (3.52) and analyze the restrictions given in (3.40), (3.41). To this end, we compute action of the operator $\mathbf{J}^{-i \dagger}$ (3.32) on the vertices (3.51), (3.52),

$$
\mathbf{J}^{-i \dagger}\left|p_{[3]}^{-}\right\rangle=\mathbf{P}^{-} \sum_{a=1,2,3} \frac{2 \check{\beta}_{a}}{3 \beta_{a}} \alpha_{a}^{i} \partial_{B_{a}}\left|p_{[3]}^{-}\right\rangle+\mathbb{P}^{i} G_{\beta}\left|p_{[3]}^{-}\right\rangle+\sum_{a=1,2,3} \frac{\alpha_{a}^{i}}{\beta_{a}} G_{a, \mathbb{P}^{2}}\left|p_{[3]}^{-}\right\rangle,
$$

where operators $G_{a, \mathbb{P}^{2}}, G_{\beta}$ are given in the appendices C, D. Using (3.40), (3.54), and explicit form of operators $G_{a, \mathbb{P}^{2}}, G_{\beta}$, it is easy to see that requiring the density $\left|j_{[3]}^{-i}\right\rangle$ to respect equations (3.40), (3.43), we get the equations (see appendices C, D)

$$
\begin{aligned}
G_{a}\left|p_{[3]}^{-}\right\rangle & =0, \quad a=1,2,3 ; \\
G_{\beta}\left|p_{[3]}^{-}\right\rangle & =0 .
\end{aligned}
$$

Using eqs. (3.40), (3.54)-(3.56) and (C.1), (D.1), we obtain the representations for the densities $\left|j_{[3]}^{-i}\right\rangle$ corresponding to the interaction vertices (3.51), (3.52),

$$
\begin{aligned}
\left|j_{[3]}^{-i}\right\rangle & =-\sum_{a=1,2,3} \frac{2 \check{\beta}_{a}}{3 \beta_{a}} \alpha_{a}^{i} \partial_{B_{a}}\left|p_{[3]}^{-}\right\rangle-\frac{2 \beta}{\beta_{3}^{3}} \frac{g_{v_{3}} \partial_{v_{3}}}{2 N_{3}+d-2} \partial_{B_{3}}^{2}\left|p_{[3]}^{-}\right\rangle, \\
\left|j_{[3]}^{-i}\right\rangle & =-\sum_{a=1,2,3} \frac{2 \check{\beta}_{a}}{3 \beta_{a}} \alpha_{a}^{i} \partial_{B_{a}}\left|p_{[3]}^{-}\right\rangle-\sum_{a=1,2} \frac{2 \beta}{\beta_{a}^{3}} \frac{g_{v_{a}} \partial_{v_{a}}}{2 N_{a}+d-2} \partial_{B_{a}}^{2}\left|p_{[3]}^{-}\right\rangle, \\
N_{a} & \equiv N_{B_{a}}+N_{\alpha_{a a+1}}+N_{\alpha_{a+2 a} a}, \quad a=1,2,3 .
\end{aligned}
$$

From (3.57), (3.58), we see that if vertices $\left|p_{[3]}^{-}\right\rangle$(3.51), (3.52) are expandable in $B_{a}$ (3.53) and satisfy eqs. (3.55), (3.56), then the respective densities $\left|j_{[3]}^{-i}\right\rangle$ (3.57), (3.58) are also expandable in the $B_{a}$.

iv) Finally, we analyze the restrictions of $J^{+-}$-symmetry (3.38). We note that, in terms of vertices given in (3.51), (3.52), eq. (3.38) is simplified as

$$
\sum_{a=1,2,3} \beta_{a} \partial_{\beta_{a}} p_{[3]}^{-}=0 .
$$

Summary of analysis of eqs. (3.38)-(3.43). To summarize the discussion in this section, we note that, in the minimal scheme, the cubic vertices describing interactions of the fields given in (3.44) and (3.45) can be cast into the form given in (3.51) and (3.52) respectively. Vertices (3.51) and (3.52) should satisfy eqs. (3.55), (3.56), (3.60), while the 
respective densities $\left|j_{[3]}^{-i}\right\rangle$ are expressed in terms of the cubic vertices $\left|p_{[3]}^{-}\right\rangle$as in (3.57) and (3.58).

Thus all that remains is to solve eqs. (3.55), (3.56), (3.60) for vertices (3.51) and (3.52) which describe the respective interactions of the fields given in (3.44) and (3.45). From now on, we separately consider solutions of eqs. (3.55), (3.56), (3.60) for vertices (3.51) and (3.52). We note also that, for vertices (3.51), there are two different cases: a) Two arbitrary spin massive fields have the same mass values; b) Two arbitrary spin massive fields have the different mass values. Structure of cubic vertices for these cases turns out to be different. We consider therefore these two cases in turn.

\section{Parity invariant cubic vertices for one continuous-spin massless field and two massive fields with the same mass values}

We start with considering parity invariant cubic vertices for one continuous-spin massless field and two arbitrary spin massive fields having the same mass values. Namely, using the shortcut $(0, \kappa)_{\mathrm{CSF}}$ for a continuous-spin massless field and the shortcut $(m, s)$ for a mass- $m$ and spin- $s$ massive field, we consider a parity invariant cubic vertices for the following three fields:

$$
\begin{aligned}
& \left(m_{1}, s_{1}\right)-\left(m_{2}, s_{2}\right)-\left(0, \kappa_{3}\right)_{\mathrm{CSF}} \quad \text { two massive fields and one continuous-spin massless field } \\
& m_{1}=m_{2}=m .
\end{aligned}
$$

Our notation in (4.1) implies that the mass- $m_{1}$, spin- $s_{1}$ massive field and the mass- $m_{2}$, spin$s_{2}$ massive field carry the respective external line indices $a=1,2$, while the continuous-spin massless field corresponds to $a=3$.

The general solution to cubic vertex for fields (4.1) takes the form (see appendix C)

$$
\begin{aligned}
p_{[3]}^{-} & =U_{v_{3}} U_{3} U_{\beta} U_{\zeta} U_{B} U_{z 2} V^{(6)}, \\
p_{[3]}^{-} & =p_{[3]}^{-}\left(\beta_{a}, B_{a}, \alpha_{a a+1}, \zeta_{1}, \zeta_{2}, v_{3}\right), \\
V^{(6)} & =V^{(6)}\left(B_{a}, \alpha_{a a+1}\right),
\end{aligned}
$$

where, in solution (4.2), we introduce new vertex $V^{(6)}$, while, in relations (4.3), (4.4), we show explicitly arguments of the generic vertex $p_{[3]}^{-}$and new vertex $V^{(6)}$. The arguments $B_{a}$ and $\alpha_{a b}$ (4.3), (4.4) are defined in (3.53). Quantities denoted by $U$ in (4.2) are differential operators w.r.t. the $B_{a}$ and $\alpha_{a a+1}$. Before presenting explicit expressions for the operators $U$ in (4.2), we note that, for vertex $V^{(6)}(4.4)$, we find two solutions given by

$$
\begin{aligned}
V^{(6)} & =\cosh \left(\Omega_{3} z_{3}\right) V, \\
V^{(6)} & =\frac{\sinh \left(\Omega_{3} z_{3}\right)}{\Omega_{3}} V, \\
V & =V\left(B_{1}, B_{2}, \alpha_{12}, \alpha_{23}, \alpha_{31}\right), \\
z_{3} & =\frac{B_{3}}{m},
\end{aligned}
$$


where, in relations (4.5), (4.6), in place of $B_{3}$ (3.53), we use a re-scaled variable $z_{3}$ (4.8) as a argument of the vertex $V^{(6)}$. In (4.7), we show explicitly arguments of the vertex $V$. The quantity $\Omega_{3}$ is a differential operator independent of $z_{3}$. This operator is defined below.

From (4.2)-(4.7), we see that general solution for the generic vertex $p_{[3]}^{-}$, which depends on the twelve variables (4.3), is expressed in terms of the vertex $V$ which is arbitrary function of the five variables (4.7). Note however that vertex $V(4.7)$ is restricted to be expandable in the five variables $B_{1}, B_{2}, \alpha_{12}, \alpha_{23}, \alpha_{31}$. Thus, the general solution for the generic vertex $p_{[3]}^{-}(4.2)$ is expressed in terms of the operators $U, \Omega_{3}$ and vertex $V$ (4.7). Therefore all that remains to complete a description of the vertex $p_{[3]}^{-}$is to provide explicit expressions for the operators $U, \Omega_{3}$. The operators $U, \Omega_{3}$ entering our solution in (4.2) are given by

$$
\begin{aligned}
U_{v_{3}} & =v_{3}^{N_{3}} \\
U_{3} & =\left(\frac{2^{N_{3}} \Gamma\left(N_{3}+\frac{d-2}{2}\right)}{\Gamma\left(N_{3}+1\right)}\right)^{1 / 2} \\
U_{\beta} & =\exp \left(-\frac{\check{\beta}_{1}}{2 \beta_{1}} m \zeta_{1} \partial_{B_{1}}-\frac{\check{\beta}_{2}}{2 \beta_{2}} m \zeta_{2} \partial_{B_{2}}-\frac{\check{\beta}_{3}}{2 \beta_{3}} \kappa_{3} \partial_{B_{3}}\right) \\
U_{\zeta} & =\exp \left(-\frac{1}{2} m \zeta_{1} \partial_{B_{1}}+\frac{1}{2} m \zeta_{2} \partial_{B_{2}}\right) \\
U_{B} & =\exp \left(\left(\frac{\zeta_{1}}{m} B_{2}-\frac{\zeta_{2}}{m} B_{1}-\zeta_{1} \zeta_{2}\right) \partial_{\alpha_{12}}-\frac{\zeta_{1}}{m}\left(B_{3}+\frac{1}{2} \kappa_{3}\right) \partial_{\alpha_{31}}+\frac{\zeta_{2}}{m}\left(B_{3}-\frac{1}{2} \kappa_{3}\right) \partial_{\alpha_{23}}\right) \\
U_{z 2} & =\exp \left(\frac{1}{2} z_{3} X+\frac{1}{4} z_{3}^{2} Y\right) \\
X & =-\frac{1}{m}\left(B_{2} \partial_{\alpha_{23}}+B_{1} \partial_{\alpha_{31}}\right) \\
Y & =\frac{2}{\kappa_{3}}\left(B_{2} \partial_{\alpha_{23}}-B_{1} \partial_{\alpha_{31}}\right) \\
Z & =2 \alpha_{12} \partial_{\alpha_{31}} \partial_{\alpha_{23}}, \\
\Omega_{3}^{2} & =1+\frac{1}{2}\left\{Y, \nu_{3}\right\}+\frac{1}{4} X^{2}+Z \\
\nu_{3} & =N_{\alpha_{23}}+N_{\alpha_{31}}+\frac{d-4}{2}
\end{aligned}
$$

where $\check{\beta}_{a}, N_{B_{a}}, N_{\alpha_{a b}}, N_{a}$ are given in (A.4)-(A.6), while $\Gamma$ (4.10) stands for the Gammafunction.

Expressions (4.2)-(4.19) provide the complete description of cubic vertices for one continuous-spin massless field and two arbitrary spin massive fields (4.1). More precisely, these cubic vertices describe an interaction of one continuous-spin massless field with two chains of totally symmetric massive fields. Each chain consists of every spin just once. Such chains of massive fields are described by ket-vectors given in (2.18). We now consider vertices for one continuous-spin massless field and two massive fields with arbitrary but fixed spin- $s_{1}$ and spin- $s_{2}$ values. Taking into account that the ket-vectors for massive fields 
$\left|\phi_{s_{a}}\right\rangle$ (2.15) are the respective degree- $s_{a}$ homogeneous polynomials in the oscillators $\alpha_{a}^{i}, \zeta_{a}$, $a=1,2,(2.16)$, it is easy to see that vertices we are interested in must satisfy the equations

$$
\left(N_{\alpha_{a}}+N_{\zeta_{a}}-s_{a}\right)\left|p_{[3]}^{-}\right\rangle=0, \quad a=1,2,
$$

which tell us that the vertices should be degree- $s_{1}$ and degree- $s_{2}$ homogeneous polynomials in the respective oscillators $\alpha_{1}^{i}, \zeta_{1}$ and $\alpha_{2}^{i}, \zeta_{2}$. Using (4.2), we verify that, in terms of $V(4.5)-(4.7)$, eqs. (4.20) take the form

$$
\begin{aligned}
& \left(N_{B_{1}}+N_{\alpha_{12}}+N_{\alpha_{31}}-s_{1}\right) V=0 \\
& \left(N_{B_{2}}+N_{\alpha_{12}}+N_{\alpha_{23}}-s_{2}\right) V=0 .
\end{aligned}
$$

As vertex $V$ (4.7) is considered to be expandable in the variables $B_{1}, B_{2}, \alpha_{12}, \alpha_{23}, \alpha_{31}$, a general solution of eqs. (4.21), (4.22) can be labelled by $s_{1}, s_{2}$ and by some three integers $n_{1}, n_{2}, n_{3}$. Using then notation $V_{s_{1}, s_{2}}\left(n_{1}, n_{2}, n_{3}\right)$ for the vertex $V$ (4.7) that satisfies eqs. (4.21), (4.22), we find the following general solution:

$$
\begin{aligned}
V & =V_{s_{1}, s_{2}}\left(n_{1}, n_{2}, n_{3}\right), \\
V_{s_{1}, s_{2}}\left(n_{1}, n_{2}, n_{3}\right) & =B_{1}^{s_{1}-n_{1}} B_{2}^{s_{2}-n_{2}} \alpha_{12}^{l_{3}} \alpha_{23}^{l_{1}} \alpha_{31}^{l_{2}}, \\
l_{1} & =\frac{1}{2}\left(-n_{1}+n_{2}+n_{3}\right), \quad l_{2}=\frac{1}{2}\left(n_{1}-n_{2}+n_{3}\right), \quad l_{3}=\frac{1}{2}\left(n_{1}+n_{2}-n_{3}\right) .
\end{aligned}
$$

Integers $n_{1}, n_{2}, n_{3}$ appearing in (4.23), (4.24) are the freedom of our solution for vertices, i.e., these integers label all possible cubic vertices that can be constructed for three fields shown in (4.1). In order for vertices (4.23) to be sensible, we should impose the following restrictions:

$$
0 \leq n_{1} \leq s_{1}, \quad 0 \leq n_{2} \leq s_{2}, \quad l_{1}, l_{2}, l_{3} \in \mathbb{N}_{0} .
$$

Restrictions (4.25) amount to the requirement that the powers of all variables $B_{1}, B_{2}, \alpha_{12}$, $\alpha_{23}, \alpha_{31}$ in (4.23) be non-negative integers. We note then that by using relations (4.24), we can rewrite restrictions (4.25) as

$$
\begin{aligned}
& \left|n_{1}-n_{2}\right| \leq n_{3} \leq n_{1}+n_{2}, \\
& 0 \leq n_{1} \leq s_{1}, \quad 0 \leq n_{2} \leq s_{2}, \\
& n_{1}+n_{2}+n_{3} \in 2 \mathbb{N}_{0}, \quad n_{1}, n_{2}, n_{3} \in \mathbb{N}_{0} .
\end{aligned}
$$

Expressions for cubic vertices in (4.2), (4.5), (4.6), (4.23) supplemented by the restrictions on allowed values of the integers $n_{1}, n_{2}, n_{3}$ given in (4.26)-(4.28) provide the complete description and classification of cubic interaction vertices that can be constructed for the one continuous-spin massless field and the two spin- $s_{1}$ and spin- $s_{2}$ massive fields having the same mass parameter (4.1).

The following remarks are in order.

i) From restrictions in (4.26), (4.27), we see that, given spin values $s_{1}$ and $s_{2}$, a number of cubic vertices that can be constructed for fields in (4.1) is finite. 
ii) From (3.53) and (4.8), we see that the variable $z_{3}$ is a degree- 1 homogeneous polynomial in the momentum $\mathbb{P}^{i}$. Taking this into account and using expressions in (4.5), (4.6), we see that vertex $p_{[3]}^{-}(4.2)$ involves all positive powers of the momentum $\mathbb{P}^{i}$. Note also that vertex $V^{(6)}(4.5)$ involves all even positive powers of the momentum $\mathbb{P}^{i}$, while vertex $V^{(6)}$ (4.6) involves all odd positive powers of the momentum $\mathbb{P}^{i}$. On the other hand, from (4.23), (4.27), we see that vertex $V(4.23)$ is finite-order polynomial in the momentum $\mathbb{P}^{i}$. Namely, vertex $V(4.23)$ is a degree- $\left(s_{1}+s_{2}-n_{1}-n_{2}\right)$ homogeneous polynomial in the $\mathbb{P}^{i}$.

iii) From $(4.23),(4.24)$, we find the relation

$$
\left(N_{\alpha_{23}}+N_{\alpha_{31}}-n_{3}\right) V_{s_{1}, s_{2}}\left(n_{1}, n_{2}, n_{3}\right)=0 .
$$

From (4.23), (4.29), we see that the vertex $V_{s_{1}, s_{2}}\left(n_{1}, n_{2}, n_{3}\right)$ is a degree- $n_{3}$ monomial in the variables $\alpha_{23}, \alpha_{31}$. From relations (4.15)-(4.19), we notice the commutators

$$
\left[\nu_{3}, X\right]=-X, \quad\left[\nu_{3}, Y\right]=-Y, \quad\left[\nu_{3}, Z\right]=-2 Z .
$$

From (4.30), it is clear that action of the operator $X^{p} Y^{q} Z^{n}$ on the vertex $V_{s_{1}, s_{2}}\left(n_{1}, n_{2}, n_{3}\right)$ gives a degree- $\left(n_{3}-p-q-2 n\right)$ homogeneous polynomial in the variables $\alpha_{23}, \alpha_{31}$. Taking this into account and using expressions for operators $U$ and $\Omega_{3}(4.9)-(4.19)$, it is easy to see that given values $s_{1}$ and $s_{2}$ the cubic vertex $p_{[3]}$ given by (4.2), (4.5), (4.6), (4.23) is a finite-order polynomial in the variables $B_{1}, B_{2}$, $\alpha_{12}, \alpha_{23}, \alpha_{31}$.

iv) Two solutions for vertex $V^{(6)}$ (4.5), (4.6) appear as follows. Equations (3.55), (3.56) lead to the following 2 nd-order differential equation for vertex $V^{(6)}$ (for details, see appendix C)

$$
\left(\partial_{z_{3}}^{2}-\Omega_{3}^{2}\right) V^{(6)}=0 .
$$

Differential equation (4.31) for the vertex $V^{(6)}$, which depends on the six variables $B_{1}, B_{2}, z_{3}, \alpha_{12}, \alpha_{23}, \alpha_{31}$ (4.4), (4.8), has two solutions presented in (4.5), (4.6), where the vertex $V$ depends on the five variables $B_{1}, B_{2}, \alpha_{12}, \alpha_{23}, \alpha_{31}(4.7)$.

Interaction of scalar massive fields and continuous-spin massless field. ${ }^{8}$ By way of example and in order to demonstrate how to use our result we consider cubic vertex for two scalar massive fields and one continuous-spin massless field. For spin values of the scalar fields, we get $s_{1}=0, s_{2}=0$, while for mass values we set $m_{1}=m, m_{2}=m$ (4.1). From (4.27), we find $n_{1}=0, n_{2}=0$. Using this in (4.26), we find $n_{3}=0$. Thus there is only one cubic vertex for two scalar massive fields and one continuous-spin massless field. Plugging values $n_{1}=0, n_{2}=0, n_{3}=0$ in (4.23), (4.24), we find $V=1$. Plugging $V=1$ in (4.5), (4.6) we find the following two vertices $V^{(6)}$ :

$$
V^{(6)}=\cosh z_{3}, \quad V^{(6)}=\sinh z_{3}, \quad z_{3}=\frac{1}{m} B_{3} .
$$

\footnotetext{
${ }^{8}$ X. Bekaert informed us that, in collaboration with J.Mourad and M.Najafizadeh, he described the minimal cubic coupling between a continuous-spin gauge field and scalar matter.
} 
Plugging (4.32) into (4.2), we get the following two interaction vertices $p_{[3]}^{-}$:

$$
\begin{aligned}
p_{[3]}^{-} & =U \cosh \left(\frac{1}{m} v_{3} B_{3}-\frac{\check{\beta}_{3} \kappa_{3}}{2 \beta_{3} m}\right), \\
p_{[3]}^{-} & =U \sinh \left(\frac{1}{m} v_{3} B_{3}-\frac{\check{\beta}_{3} \kappa_{3}}{2 \beta_{3} m}\right), \\
U & =\left(\frac{2^{B_{3}} \Gamma\left(N_{B_{3}}+\frac{d-2}{2}\right)}{\Gamma\left(N_{B_{3}}+1\right)}\right)^{1 / 2}, \quad N_{B_{3}}=B_{3} \partial_{B_{3}} .
\end{aligned}
$$

Vertex (4.33) is symmetric upon the replacement of external line indices of scalar fields, $1 \leftrightarrow 2$, and this vertex describes interaction of two scalar massive fields with one continuousspin massless field. In the limit $\kappa_{3} \rightarrow 0$, vertex (4.33) is decomposed into a direct sum of vertices which describe interactions of two scalar massive fields with massless fields having even spin values. Vertex (4.34) is anti-symmetric upon the replacement of external line indices of scalar fields, $1 \leftrightarrow 2$, and this vertex also describes interaction of two scalar massive fields with one continuous-spin massless field. In the limit $\kappa_{3} \rightarrow 0$, vertex (4.34) is decomposed into a direct sum of vertices which describe interactions of two scalar massive fields with massless fields having odd spin values. ${ }^{9}$

v) We see that vertices (4.33), (4.34) are singular in the limit $m \rightarrow 0$. This implies that there are no cubic vertices describing consistent interaction of one continuous-spin massless field with two scalar massless fields. In appendix E, we demonstrate that, contrary to the cubic vertices for three arbitrary spin massless fields, cubic vertices for one continuous-spin massless field and two arbitrary spin massless fields are not consistent. ${ }^{10}$

vi) We describe symmetry properties of various quantities and operators entering our solution (4.2). Upon the replacement of the external line indices of arbitrary spin massive fields, $1 \leftrightarrow 2$, the quantities $\check{\beta}_{a}, \mathbb{P}^{i}(3.17)$ and $B_{a}(3.53)$ are changed as

$$
\check{\beta}_{1} \leftrightarrow-\check{\beta}_{2}, \quad \check{\beta}_{3} \leftrightarrow-\check{\beta}_{3}, \quad \mathbb{P}^{i} \leftrightarrow-\mathbb{P}^{i}, \quad B_{1} \leftrightarrow-B_{2}, \quad B_{3} \leftrightarrow-B_{3} .
$$

Using (4.36), we note then the behaviour of quantities in (4.15)-(4.19) upon the replacement of the external line indices of arbitrary spin massive fields, $1 \leftrightarrow 2$,

$$
X \leftrightarrow X, \quad Y \leftrightarrow-Y, \quad Z \leftrightarrow Z, \quad z_{3} \leftrightarrow-z_{3}, \quad \Omega_{3}^{2} \leftrightarrow \Omega_{3}^{2}, \quad \nu_{3} \leftrightarrow \nu_{3} .
$$

\footnotetext{
${ }^{9}$ In order to get non-trivial interaction for vertices in (4.34) one needs, as usually, to introduce internal symmetry. Incorporation of the internal symmetry into the game can be done via the Chan-Paton method in string theory [35], and could be performed as in ref. [36].

${ }^{10}$ In light-cone gauge approach, parity invariant cubic vertices for three arbitrary spin massless fields in $R^{d-1,1}, d \geq 4$, were obtained in ref. [31]. In BRST approach, such vertices were studied in refs. [37, 38], while, in metric-like approach, in refs. [39-41]. In BRST approach, cubic vertices involving arbitrary spin massless and massive fields were derived in ref. [31]. Interesting discussion of BRST approach may be found in refs. [42-44].
} 
Relations (4.36), (4.37) imply that all operators $U$ (4.9)-(4.14) are symmetric upon the replacement of external line indices of arbitrary spin massive fields, $1 \leftrightarrow 2$,

$$
U_{v_{3}} \leftrightarrow U_{v_{3}}, \quad U_{3} \leftrightarrow U_{3}, \quad U_{\beta} \leftrightarrow U_{\beta}, \quad U_{\zeta} \leftrightarrow U_{\zeta}, \quad U_{B} \leftrightarrow U_{B}, \quad U_{z 2} \leftrightarrow U_{z 2} .
$$

\section{Parity invariant cubic vertices for one continuous-spin massless field and two arbitrary spin massive fields with the different mass values}

In this section, we consider parity invariant cubic vertices for one continuous-spin massless field and two arbitrary spin massive fields having different mass values. Namely, using the shortcut $(0, \kappa)_{\mathrm{CSF}}$ for continuous-spin massless field and the shortcut $(m, s)$ for mass- $m$ and spin- $s$ massive field, we consider parity invariant cubic vertices for the following three fields:

$$
\begin{aligned}
& \left(m_{1}, s_{1}\right)-\left(m_{2}, s_{2}\right)-\left(0, \kappa_{3}\right)_{\mathrm{CSF}} \quad \text { two massive fields and one continuous-spin massless field } \\
& m_{1} \neq m_{2} .
\end{aligned}
$$

Our notation in (5.1) implies that mass- $m_{1}$, spin- $s_{1}$ and mass- $m_{2}$, spin- $s_{2}$ massive fields carry the respective external line indices $a=1,2$, while the continuous-spin massless field corresponds to $a=3$.

The general solution to cubic vertex for fields in (5.1) takes the form (see appendix C)

$$
\begin{aligned}
p_{[3]}^{-} & =U_{v_{3}} U_{3} U_{\beta} U_{\zeta} U_{B} U_{z 1} U_{z \nu} U_{W} V^{(8)}, \\
p_{[3]}^{-} & =p_{[3]}^{-}\left(\beta_{a}, B_{a}, \alpha_{a a+1}, \zeta_{1}, \zeta_{2}, v_{3}\right), \\
V^{(8)} & =V^{(8)}\left(B_{a}, \alpha_{a a+1}\right),
\end{aligned}
$$

where, in the solution (5.2), we introduce new vertex denoted by $V^{(8)}$, while, in relations (5.3), (5.4), we show explicitly arguments of the generic vertex $p_{[3]}^{-}$and the new vertex $V^{(8)}$. Before presenting operators $U$ appearing in (5.2) we note that, for vertex $V^{(8)}(5.4)$, we find two solutions which can be expressed in terms of the modified Bessel functions,

$$
\begin{aligned}
V^{(8)} & =I_{\nu_{3}}\left(\sqrt{4 z_{3}}\right) V, \\
V^{(8)} & =K_{\nu_{3}}\left(\sqrt{4 z_{3}}\right) V, \\
V & =V\left(B_{1}, B_{2}, \alpha_{12}, \alpha_{23}, \alpha_{31}\right), \\
z_{3} & =\frac{\kappa_{3}^{2}\left(m_{1}^{2}+m_{2}^{2}\right)}{2\left(m_{1}^{2}-m_{2}^{2}\right)^{2}}-\frac{\kappa_{3}}{m_{1}^{2}-m_{2}^{2}} B_{3}, \\
\nu_{3} & =N_{\alpha_{23}}+N_{\alpha_{31}}+\frac{d-4}{2},
\end{aligned}
$$

where $B_{a}, N_{\alpha_{a b}}$ are given in (A.4), (A.5). In (5.5), (5.6), in place of the $B_{3}$ (3.53), we use a new variable $z_{3}(5.8)$ as a argument of the vertex $V^{(8)}$. For the modified Bessel functions $I_{\nu}$ and $K_{\nu}$ (5.5), (5.6), we use conventions in ref. [45].

From (5.3), we see that the vertex $p_{[3]}^{-}$depends on twelve variables, while, from (5.7), we learn that the vertex $V$ depends on five variables. Vertex $V(5.7)$ is restricted to be 
expandable in the five variables $B_{1}, B_{2}, \alpha_{12}, \alpha_{23}, \alpha_{31}$. The general solution for vertex $p_{[3]}^{-}(5.2)$ is expressed in terms of the operators $U$, operator $\nu_{3}(5.9)$, and vertex $V(5.7)$. All that remains to complete the description of the solution for the vertex $p_{[3]}^{-}$in (5.2) is to provide explicit expressions for the operators $U$. The operators $U$ appearing in (5.2) are given by

$$
\begin{aligned}
U_{v_{3}} & =v_{3}^{N_{3}}, N_{3} \equiv N_{B_{3}}+N_{\alpha_{31}}+N_{\alpha_{23}}, \\
U_{3} & =\left(\frac{2^{N_{3}} \Gamma\left(N_{3}+\frac{d-2}{2}\right)}{\Gamma\left(N_{3}+1\right)}\right)^{1 / 2}, \\
U_{\beta} & =\exp \left(-\frac{\check{\beta}_{1}}{2 \beta_{1}} m_{1} \zeta_{1} \partial_{B_{1}}-\frac{\check{\beta}_{2}}{2 \beta_{2}} m_{2} \zeta_{2} \partial_{B_{2}}-\frac{\check{\beta}_{3}}{2 \beta_{3}} \kappa_{3} \partial_{B_{3}}\right), \\
U_{\zeta} & =\exp \left(-\frac{m_{2}^{2}}{2 m_{1}} \zeta_{1} \partial_{B_{1}}+\frac{m_{1}^{2}}{2 m_{2}} \zeta_{2} \partial_{B_{2}}\right) \\
U_{B} & =\exp \left(\left(\frac{\zeta_{1}}{m_{1}} B_{2}-\frac{\zeta_{2}}{m_{2}} B_{1}-\frac{m_{1}^{2}+m_{2}^{2}}{2 m_{1} m_{2}} \zeta_{1} \zeta_{2}\right) \partial_{\alpha_{12}}\right. \\
U_{z 1} & =\exp \left(-X+z_{3} Y\right), \\
U_{z \nu} & \left.\left.=z_{3}^{-\nu_{3} / 2}, B_{3}+\frac{1}{2} \kappa_{3}\right) \partial_{\alpha_{31}}+\frac{\zeta_{2}}{m_{2}}\left(B_{3}-\frac{1}{2} \kappa_{3}\right) \partial_{\alpha_{23}}\right), \\
U_{W} & =\sum_{n=0}^{\infty} \frac{\Gamma\left(\nu_{3}+n\right)}{n ! \Gamma\left(\nu_{3}+2 n\right)} W^{n}, \\
X & =\frac{2 \kappa_{3}}{\left(m_{1}^{2}-m_{2}^{2}\right)^{2}}\left(m_{1}^{2} B_{1} \partial_{\alpha_{31}}-m_{2}^{2} B_{2} \partial_{\alpha_{23}}\right), \\
Y & =\frac{2}{\kappa_{3}}\left(B_{2} \partial_{\alpha_{23}}-B_{1} \partial_{\alpha_{31}}\right), \\
Z & =2 \alpha_{12} \partial_{\alpha_{31}} \partial_{\alpha_{23}}, \\
W & =Z+X Y,
\end{aligned}
$$

where $\check{\beta}_{a}, N_{B_{a}}, N_{\alpha_{a b}}, \nu_{a}, N_{a}$ are defined in (A.4)-(A.6), while the symbol $\Gamma(5.11),(5.17)$ stands for the Gamma-function.

Expressions in (5.2)-(5.21) provide the complete description of the cubic vertex describing interaction of one continuous-spin massless field with two infinite chains of arbitrary spin massive fields. We recall that the infinite chain of massive fields is described by ketvector given in (2.18). In the chain of massive fields carrying external line index $a=1$, all fields have mass value $m_{1}$, while, in the chain of massive fields carrying external line index $a=2$, all fields have mass value $m_{2}, m_{1} \neq m_{2}$. To consider vertices for one continuous-spin massless field and arbitrary but fixed spin- $s_{1}$ and spin- $s_{2}$ massive fields (5.1) we note that the ket-vectors for massive spin- $s_{a}$ fields $\left|\phi_{s_{a}}\right\rangle$ are the respective degree- $s_{a}$ homogeneous polynomials in the oscillators $\alpha_{a}^{i}, \zeta_{a}, a=1,2,(2.16)$. This implies that the vertices we are interested in must satisfy the equations

$$
\left(N_{\alpha_{a}}+N_{\zeta_{a}}-s_{a}\right)\left|p_{[3]}^{-}\right\rangle=0, \quad a=1,2,
$$


which tell us that the $\left|p_{[3]}^{-}\right\rangle$should be degree- $s_{1}$ and degree- $s_{2}$ homogeneous polynomial in the respective oscillators $\alpha_{1}^{i}, \zeta_{1}$ and $\alpha_{2}^{i}, \zeta_{2}$. Using (5.2), we verify that, in terms of vertex $V(5.5),(5.6)$, eqs. (5.22) take the form

$$
\begin{aligned}
& \left(N_{B_{1}}+N_{\alpha_{12}}+N_{\alpha_{31}}-s_{1}\right) V=0 \\
& \left(N_{B_{2}}+N_{\alpha_{12}}+N_{\alpha_{23}}-s_{2}\right) V=0 .
\end{aligned}
$$

Vertex $V(5.7)$ is restricted to be expandable in the variables $B_{1}, B_{2}, \alpha_{12}, \alpha_{23}, \alpha_{31}$. Therefore solution of eqs. (5.23), (5.24) can be labelled by spin values $s_{1}, s_{2}$ and by some three integers $n_{1}, n_{2}, n_{3}$. Using the notation $V_{s_{1}, s_{2}}\left(n_{1}, n_{2}, n_{3}\right)$ for vertex $V(5.7)$ that satisfies eqs. (5.23), (5.24), we find the following general solution:

$$
\begin{aligned}
V & =V_{s_{1}, s_{2}}\left(n_{1}, n_{2}, n_{3}\right), \\
V_{s_{1}, s_{2}}\left(n_{1}, n_{2}, n_{3}\right) & =B_{1}^{s_{1}-n_{1}} B_{2}^{s_{2}-n_{2}} \alpha_{12}^{l_{3}} \alpha_{23}^{l_{1}} \alpha_{31}^{l_{2}}, \\
l_{1} & =\frac{1}{2}\left(-n_{1}+n_{2}+n_{3}\right), \quad l_{2}=\frac{1}{2}\left(n_{1}-n_{2}+n_{3}\right), \quad l_{3}=\frac{1}{2}\left(n_{1}+n_{2}-n_{3}\right) .
\end{aligned}
$$

Integers $n_{1}, n_{2}, n_{3}$ in (5.25), (5.26) are the freedom of our solution for vertices, i.e., these integers label all possible cubic vertices that can be constructed for fields in (5.1). In order for vertices (5.25) to be sensible, we should impose the following restrictions:

$$
0 \leq n_{1} \leq s_{1}, \quad 0 \leq n_{2} \leq s_{2}, \quad l_{1}, l_{2}, l_{3} \in \mathbb{N}_{0},
$$

which amount to the requirement that the powers of variables $B_{1}, B_{2}, \alpha_{12}, \alpha_{23}, \alpha_{31}$ in (5.25) be non-negative integers. Using relations (5.26), we represent restrictions (5.27) as

$$
\begin{aligned}
& \left|n_{1}-n_{2}\right| \leq n_{3} \leq n_{1}+n_{2}, \\
& 0 \leq n_{1} \leq s_{1}, \quad 0 \leq n_{2} \leq s_{2}, \\
& n_{1}+n_{2}+n_{3} \in 2 \mathbb{N}_{0}, \quad n_{1}, n_{2}, n_{3} \in \mathbb{N}_{0} .
\end{aligned}
$$

Relations in (5.2), (5.5), (5.6), (5.25) and restrictions in (5.28)-(5.30) provide the complete description and classification of cubic vertices that can be constructed for one continuousspin massless field and two spin- $s_{1}$ and spin- $s_{2}$ massive fields having different mass parameters (5.1).

The following remarks are in order.

i) From restrictions in (5.28), (5.29), we see that given spin values $s_{1}$ and $s_{2}$ a number of cubic vertices that can be constructed for fields in (5.1) is finite.

ii) From (3.53) and (5.8), we see that the variable $z_{3}$ is degree-1 polynomial in the momentum $\mathbb{P}^{i}$. Taking this into account and using expressions in (5.5), (5.6), we see that vertex $p_{[3]}^{-}(5.2)$ involves all positive powers of the momentum $\mathbb{P}^{i}$. On the other hand, from (5.25), (5.29), we see that vertex $V$ (5.25) is finite-order polynomial in the momentum $\mathbb{P}^{i}$. Namely, vertex $V(5.25)$ is a degree- $\left(s_{1}+s_{2}-n_{1}-n_{2}\right)$ homogeneous polynomial in the momentum $\mathbb{P}^{i}$. 
iii) From (5.25), (5.26), we find the relation

$$
\left(N_{\alpha_{23}}+N_{\alpha_{31}}-n_{3}\right) V_{s_{1}, s_{2}}\left(n_{1}, n_{2}, n_{3}\right)=0
$$

From (5.25), (5.31), we see that the vertex $V_{s_{1}, s_{2}}\left(n_{1}, n_{2}, n_{3}\right)$ is a degree- $n_{3}$ monomial in the variables $\alpha_{23}, \alpha_{31}$. Using (5.9) and (5.18)-(5.21), we note the commutators

$$
\left[\nu_{3}, X\right]=-X, \quad\left[\nu_{3}, Y\right]=-Y, \quad\left[\nu_{3}, Z\right]=-2 Z, \quad\left[\nu_{3}, W\right]=-2 W .
$$

From (5.32), it is clear that action of the operator $X^{p} Y^{q} Z^{n} W^{m}$ on the vertex $V_{s_{1}, s_{2}}\left(n_{1}, n_{2}, n_{3}\right)$ gives a degree- $\left(n_{3}-p-q-2 n-2 m\right)$ homogeneous polynomial in the variables $\alpha_{23}, \alpha_{31}$. Taking this into account and using expressions for operators $U(5.10)-(5.21)$, it is easy to see that given $s_{1}$ and $s_{2}$ the cubic vertex $p_{[3]}$ given by (5.2), (5.5), (5.6), (5.25) is a finite-order polynomial in the variables $B_{1}, B_{2}, \alpha_{12}$, $\alpha_{23}, \alpha_{31}$.

iv) Two solutions for vertex $V^{(8)}(5.5),(5.6)$ appear as follows. Equations (3.55), (3.56) lead to the following 2nd-order differential equation for the vertex $V^{(8)}$ (for details, see appendix C)

$$
\left(1-\left(N_{z_{3}}+1\right) \partial_{z_{3}}+\frac{\nu_{3}^{2}}{4 z_{3}}\right) V^{(8)}=0, \quad N_{z_{3}} \equiv z_{3} \partial_{z_{3}} .
$$

Differential equation (5.33) for the vertex $V^{(8)}$, which depends on the six variables $B_{1}, B_{2}, z_{3}, \alpha_{12}, \alpha_{23}, \alpha_{31}$ (5.4), (5.8), has two solutions presented in (5.5), (5.6), where the vertex $V$ depends on the five variables $B_{1}, B_{2}, \alpha_{12}, \alpha_{23}, \alpha_{31}(5.7)$.

v) Using symmetry properties (4.36) of quantities $\check{\beta}_{a}, \mathbb{P}^{i}$ (3.17) upon the replacement of external line indices of arbitrary spin massive fields, $1 \leftrightarrow 2$, we verify behavior of quantities in (5.8), (5.9) and (5.18)-(5.21) upon the replacement $1 \leftrightarrow 2$,

$$
z_{3} \leftrightarrow z_{3}, \quad \nu_{3} \leftrightarrow \nu_{3}, \quad X \leftrightarrow X, \quad Y \leftrightarrow Y, \quad Z \leftrightarrow Z, \quad W \leftrightarrow W
$$

Also, using (4.36) and (5.34), we verify that all operators $U$ (5.10)-(5.17) are symmetric upon the replacement of external line indices of arbitrary spin massive fields, $1 \leftrightarrow 2$,

$$
\begin{array}{rlrl}
U_{v_{3}} & \leftrightarrow U_{v_{3}}, \\
U_{z 1} & \leftrightarrow U_{z 1}, & U_{3} & \leftrightarrow U_{3}, \\
U_{z \nu} & \leftrightarrow U_{z \nu}, & U_{\beta} & \leftrightarrow U_{\beta}, \\
U_{W} & \leftrightarrow U_{W} .
\end{array}
$$

\section{Parity invariant cubic vertices for two continuous-spin massless fields and one arbitrary spin massive field}

In this section, we consider parity invariant cubic vertices for two continuous-spin massless fields and one arbitrary spin massive field. Namely, using the shortcut $(0, \kappa)_{\mathrm{CSF}}$ for 
continuous-spin massless field and the shortcut $(m, s)$ for spin-s massive field with mass parameter $m$, we consider cubic interaction vertices for the following three fields:

$$
\left(0, \kappa_{1}\right)_{\mathrm{CSF}^{-}}\left(0, \kappa_{2}\right)_{\mathrm{CSF}^{-}}\left(m_{3}, s_{3}\right) \quad \text { two continuous-spin massless fields and one massive field. }
$$

Our notation in (6.1) implies that two continuous-spin massless fields denoted by $\left(0, \kappa_{1}\right)_{\mathrm{CSF}}$ and $\left(0, \kappa_{2}\right)_{\mathrm{CSF}}$ carry the respective external line indices $a=1,2$, while the mass- $m_{3}$, spin- $s_{3}$ massive field denoted by $\left(m_{3}, s_{3}\right)$ carries external line index $a=3$.

The general solution to cubic vertex for fields in (6.1) takes the form (see appendix D)

$$
\begin{aligned}
p_{[3]}^{-} & =U_{v_{1}, v_{2}} U_{1,2} U_{\beta} U_{B} U_{z 0} U_{z 2} U_{z \nu} U_{W} V^{(8)}, \\
p_{[3]}^{-} & =p_{[3]}^{-}\left(\beta_{a}, B_{a}, \alpha_{a a+1}, v_{1}, v_{2}, \zeta_{3}\right), \\
V^{(8)} & =V^{(8)}\left(B_{a}, \alpha_{a a+1}\right)
\end{aligned}
$$

where, in the solution (6.2), we introduce new vertex denoted by $V^{(8)}$, while, in relations (6.3), (6.4), we show explicitly arguments of the generic vertex $p_{[3]}^{-}$and the new vertex $V^{(8)}$. Before presenting operators $U$ appearing in (6.2), we note that, for vertex $V^{(8)}(6.4)$, we find four solutions which can be expressed in terms of the modified Bessel functions,

$$
\begin{aligned}
V^{(8)} & =I_{\nu_{1}}\left(\sqrt{4 z_{1}}\right) I_{\nu_{2}}\left(\sqrt{4 z_{2}}\right) V, \\
V^{(8)} & =I_{\nu_{1}}\left(\sqrt{4 z_{1}}\right) K_{\nu_{2}}\left(\sqrt{4 z_{2}}\right) V, \\
V^{(8)} & =K_{\nu_{1}}\left(\sqrt{4 z_{1}}\right) I_{\nu_{2}}\left(\sqrt{4 z_{2}}\right) V, \\
V^{(8)} & =K_{\nu_{1}}\left(\sqrt{4 z_{1}}\right) K_{\nu_{2}}\left(\sqrt{4 z_{2}}\right) V, \\
V & =V\left(B_{3}, \alpha_{12}, \alpha_{23}, \alpha_{31}\right), \\
z_{1} & =\frac{\kappa_{1}}{m_{3}^{2}}\left(B_{1}+\frac{\kappa_{1}}{2}\right), \\
z_{2} & =\frac{\kappa_{2}}{m_{3}^{2}}\left(-B_{2}+\frac{\kappa_{2}}{2}\right), \\
\nu_{1} & =N_{\alpha_{12}}+N_{\alpha_{31}}+\frac{d-4}{2}, \\
\nu_{2} & =N_{\alpha_{12}}+N_{\alpha_{23}}+\frac{d-4}{2},
\end{aligned}
$$

where $B_{a}, N_{\alpha_{a b}}$ are defined in (A.4), (A.5). In (6.5)-(6.8), in place of the $B_{1}$ and $B_{2}$, we use new respective variables $z_{1}$ and $z_{2}$ (6.10), (6.11). In relations (6.5)-(6.8), the $I_{\nu}$ and $K_{\nu}$ stand for the modified Bessel functions. For the modified Bessel functions, we use conventions in ref. [45].

From (6.2)-(6.13), we see that the general solution for the vertex $p_{[3]}^{-}$, which depends on twelve variables (6.3), is expressed in terms of the vertex $V$ which is arbitrary function of four variables (6.9). Note that vertex $V(6.9)$ is restricted to be expandable in the four variables $B_{3}, \alpha_{12}, \alpha_{23}, \alpha_{31}$. Thus, the general solution for vertex $p_{[3]}^{-}(6.2)$ is expressed in terms of the operators $U, \nu_{1}, \nu_{2}$ and the vertex $V(6.9)$. Therefore all that remains to complete a description of the vertex $p_{[3]}^{-}$is to provide explicit expressions for the operators 
$U$. The operators $U$ entering our solution in (6.2) are given by

$$
\begin{aligned}
& U_{v_{1}, v_{2}}=v_{1}^{N_{1}} v_{2}^{N_{2}} \\
& N_{1}=N_{B_{1}}+N_{\alpha_{12}}+N_{\alpha_{31}}, \quad N_{2}=N_{B_{2}}+N_{\alpha_{12}}+N_{\alpha_{23}}, \\
& U_{1,2}=\left(\frac{2^{N_{1}} \Gamma\left(N_{1}+\frac{d-2}{2}\right)}{\Gamma\left(N_{1}+1\right)} \frac{2^{N_{2}} \Gamma\left(N_{2}+\frac{d-2}{2}\right)}{\Gamma\left(N_{2}+1\right)}\right)^{1 / 2}, \\
& U_{\beta}=\exp \left(-\frac{\check{\beta}_{1}}{2 \beta_{1}} \kappa_{1} \partial_{B_{1}}-\frac{\check{\beta}_{2}}{2 \beta_{2}} \kappa_{2} \partial_{B_{2}}-\frac{\check{\beta}_{3}}{2 \beta_{3}} m_{3} \zeta_{3} \partial_{B_{3}}\right) \text {, } \\
& U_{\zeta}=\exp \left(\frac{\zeta_{3}}{m_{3}}\left(B_{1}-\frac{1}{2} \kappa_{1}\right) \partial_{\alpha_{31}}-\frac{\zeta_{3}}{m_{3}}\left(B_{2}+\frac{1}{2} \kappa_{2}\right) \partial_{\alpha_{23}}\right) \\
& U_{z 0}=\exp \left(\frac{2 \kappa_{1} \kappa_{2}}{m_{3}^{2}} \partial_{\alpha_{12}}+\frac{2 \kappa_{1}}{m_{3}^{2}} B_{3} \partial_{\alpha_{31}}-\frac{2 \kappa_{2}}{m_{3}^{2}} B_{3} \partial_{\alpha_{23}}\right) \text {, } \\
& U_{z 2}=\exp \left(\frac{2 m_{3}^{2}}{\kappa_{1} \kappa_{2}} z_{1} z_{2} \partial_{\alpha_{12}}+\frac{2}{\kappa_{1}} z_{1} B_{3} \partial_{\alpha_{31}}-\frac{2}{\kappa_{2}} z_{2} B_{3} \partial_{\alpha_{23}}\right) \text {, } \\
& U_{z \nu}=z_{1}^{-\nu_{1} / 2} z_{2}^{-\nu_{2} / 2} \text {, } \\
& U_{W}=\sum_{n=0}^{\infty} U_{n}, \quad U_{n}=\sum_{m=0}^{n} U_{n-m, m}, \\
& U_{n, m}=\frac{\Gamma\left(\nu_{1}+n\right)}{\Gamma\left(\nu_{1}+2 n\right)} \sum_{k=0}^{\min (n, m)} \frac{1}{k !(n-k) !} W_{1,0}^{n-k} W_{1,1}^{k} U_{0, m-k}, \\
& U_{0, m}=\frac{\Gamma\left(\nu_{2}+m\right)}{m ! \Gamma\left(\nu_{2}+2 m\right)} W_{0,1}^{m}, \\
& W_{1,0}=2 \alpha_{23} \partial_{\alpha_{12}} \partial_{\alpha_{31}}-\frac{4}{m_{3}^{2}} B_{3}^{2} \partial_{\alpha_{31}}^{2}, \\
& W_{1,1}=-4 \partial_{\alpha_{12}}^{2}, \\
& W_{0,1}=2 \alpha_{31} \partial_{\alpha_{12}} \partial_{\alpha_{23}}-\frac{4}{m_{3}^{2}} B_{3}^{2} \partial_{\alpha_{23}}^{2} \text {, }
\end{aligned}
$$

where $\check{\beta}_{a}, N_{B_{a}}, N_{\alpha_{a b}}, \nu_{a}, N_{a}$ are defined in (A.4)-(A.6), while the $\Gamma$ (6.16)-(6.24) stands for the Gamma-function.

Expressions given in (6.2)-(6.27) provide the complete description of the cubic vertex describing interaction of two continuous-spin massless fields with one infinite chain of massive fields. The chain of massive fields consists of every spin just once. Such chain of massive fields is described by ket-vector (2.18). To consider vertices for two continuousspin massless fields and one arbitrary but fixed spin- $s_{3}$ massive field (6.1), we note that the ket-vector for massive field $\left|\phi_{s_{3}}\right\rangle$ is a degree- $s_{3}$ homogeneous polynomial in the oscillators $\alpha_{3}^{i}, \zeta_{3}(2.16)$. This implies that the vertices we are interested in must satisfy the equation

$$
\left(N_{\alpha_{3}}+N_{\zeta_{3}}-s_{3}\right)\left|p_{[3]}^{-}\right\rangle=0
$$

which tells us that the $\left|p_{[3]}^{-}\right\rangle$should be degree- $s_{3}$ homogeneous polynomial in the oscillators $\alpha_{3}^{i}, \zeta_{3}$. Using (6.2), we verify that, in terms of vertex $V(6.5)-(6.9)$, eq. (6.28) takes the 
form

$$
\left(N_{B_{3}}+N_{\alpha_{23}}+N_{\alpha_{31}}-s_{3}\right) V=0 .
$$

Vertex $V(6.9)$ is considered to be expandable in the variables $B_{3}, \alpha_{12}, \alpha_{23}, \alpha_{31}$. Therefore solution of eq. (6.29) for the vertex $V$ can be labelled by $s_{3}$ and by three integers $n_{1}, n_{2}$, $n_{3}$. Using the notation $V_{s_{3}}\left(n_{1}, n_{2}, n_{3}\right)$ for vertex $V$ (6.9) that satisfies eq. (6.29), we find the general solution,

$$
\begin{aligned}
V & =V_{s_{3}}\left(n_{1}, n_{2}, n_{3}\right), \\
V_{s_{3}}\left(n_{1}, n_{2}, n_{3}\right) & =B_{3}^{s_{3}-n_{1}-n_{2}} \alpha_{12}^{n_{3}} \alpha_{23}^{n_{1}} \alpha_{31}^{n_{2}}, \\
n_{1}, n_{2}, n_{3} & =0,1,2, \ldots, \infty .
\end{aligned}
$$

Integers $n_{1}, n_{2}, n_{3}$ in (6.30) are the freedom of our solution for vertices, i.e., these integers label all possible cubic vertices that can be constructed for fields in (6.1). In order for vertices (6.30) to be sensible, we should impose the following restrictions:

$$
n_{1}+n_{2} \leq s_{3}, \quad n_{1}, n_{2}, n_{3} \in \mathbb{N}_{0},
$$

which amount to the requirement that the powers of variables $B_{3}, \alpha_{12}, \alpha_{23}, \alpha_{31}$ in (6.30) be non-negative integers. Relations in (6.2), (6.5)-(6.9), (6.30) and restrictions in (6.32) provide the complete description and classification of cubic vertices that can be constructed for two continuous-spin massless fields and one mass- $m_{3}$, spin- $s_{3}$ massive field.

The following remarks are in order.

i) Given $s_{3}$, the integers $n_{1}$ and $n_{2}$ take finite number of values (6.32), while the integer $n_{3}$ takes infinite number of values, $n_{3} \in \mathbb{N}_{0}$ (6.32). This implies that, given $s_{3}$, a number of cubic vertices that can be constructed for fields (6.1) is infinite.

ii) From (3.53) and $(6.10),(6.11)$, we see that the variables $z_{1}, z_{2}$ are degree-1 polynomials in the momentum $\mathbb{P}^{i}$. Taking this into account and using (6.5)-(6.8), we see that vertex $p_{[3]}^{-}(6.2)$ involves all positive powers of the momentum $\mathbb{P}^{i}$. On the other hand, from (6.30), (6.32), we see that, given $s_{3}$, vertex $V(6.30)$ is finite-order polynomial in the momentum $\mathbb{P}^{i}$. Namely, vertex $V(6.30)$ is a degree- $\left(s_{3}-n_{1}-n_{2}\right)$ homogeneous polynomial in $\mathbb{P}^{i}$.

iii) Vertex $V(6.30)$ is a finite-order polynomial in the variables $B_{3}, \alpha_{12}, \alpha_{23}, \alpha_{31}$. We now show that vertex $p_{[3]}^{-}(6.2)$ is also a finite-order polynomial in the variables $B_{3}$, $\alpha_{12}, \alpha_{23}, \alpha_{31}$. To this end we note that operators $\nu_{1}, \nu_{2}$ (6.12), (6.13) and operators 
$W_{1,0}, W_{1,1} W_{0,1}(6.25)-(6.27)$ satisfy the following commutation relations

$$
\begin{aligned}
{\left[\nu_{1}, W_{1,0}\right] } & =-2 W_{1,0}, \\
{\left[\nu_{2}, W_{1,0}\right] } & =0 \\
{\left[\nu_{1}, W_{0,1}\right] } & =0 \\
{\left[\nu_{2}, W_{0,1}\right] } & =-2 W_{0,1}, \\
{\left[\nu_{1}, W_{1,1}\right] } & =-2 W_{1,1}, \\
{\left[\nu_{2}, W_{1,1}\right] } & =-2 W_{1,1}, \\
{\left[W_{1,0}, W_{0,1}\right] } & =\left(\nu_{1}-\nu_{2}\right) W_{1,1} .
\end{aligned}
$$

Using operator $U_{n, m}(6.23)$ and commutators (6.33)-(6.38), we find the commutators

$$
\begin{aligned}
& {\left[\nu_{1}, U_{n, m}\right]=-2 n U_{n, m},} \\
& {\left[\nu_{2}, U_{n, m}\right]=-2 m U_{n, m} .}
\end{aligned}
$$

Now using operator $U_{n}$ (6.22) and commutators (6.40), (6.41), we see that, for a sufficiently large number $N$, action of the operator $U_{n}$ on the vertex $V$ (6.30) gives zero for all $n \geq N$. This implies that an action of operator $U_{W}(6.22)$ on vertex $V$ (6.30) gives finite-order polynomial in the variables $B_{3}, \alpha_{12}, \alpha_{23}, \alpha_{31}$. Taking this into account and using (6.14)-(6.21) it is clear that vertex $p_{[3]}^{-}(6.2)$ is also a finiteorder polynomial in the variables $B_{3}, \alpha_{12}, \alpha_{23}, \alpha_{31}$. For the reader convenience, we note that operator $U_{n, m}(6.23)$ can equivalently be represented as

$$
\begin{aligned}
U_{n, m} & =\frac{\Gamma\left(\nu_{2}+m\right)}{\Gamma\left(\nu_{2}+2 m\right)} \sum_{k=0}^{\min (n, m)} \frac{1}{k !(m-k) !} W_{0,1}^{m-k} W_{1,1}^{k} U_{n-k, 0}, \\
U_{n, 0} & =\frac{\Gamma\left(\nu_{1}+n\right)}{n ! \Gamma\left(\nu_{1}+2 n\right)} W_{1,0}^{n} .
\end{aligned}
$$

iv) Four solutions for vertex $V^{(8)}(6.5)-(6.8)$ are obtained as follows. Equations (3.55), (3.56) lead to the following two second-order equations for the vertex $V^{(8)}$ (for details, see appendix D):

$$
\begin{array}{ll}
\left(1-\left(N_{z_{1}}+1\right) \partial_{z_{1}}+\frac{\nu_{1}^{2}}{4 z_{1}}\right) V^{(8)}=0, & N_{z_{1}} \equiv z_{1} \partial_{z_{1}}, \\
\left(1-\left(N_{z_{2}}+1\right) \partial_{z_{2}}+\frac{\nu_{2}^{2}}{4 z_{2}}\right) V^{(8)}=0, & N_{z_{2}} \equiv z_{2} \partial_{z_{2}} .
\end{array}
$$

Differential equations (6.44), (6.45) for the vertex $V^{(8)}$ depending on the six variables $z_{1}, z_{2}, B_{3}, \alpha_{12}, \alpha_{23}, \alpha_{31}(6.4),(6.10)$, (6.11) have four solutions presented in (6.5), (6.6), where the vertex $V$ depends on the four variables $B_{3}, \alpha_{12}, \alpha_{23}$, $\alpha_{31}(6.9)$.

v) Using symmetry properties (4.36) of quantities $\check{\beta}_{a}, \mathbb{P}^{i}(3.17)$ upon the replacement of external line indices of continuous-spin massless fields, $1 \leftrightarrow 2$, we verify behavior of 
quantities in (6.10)-(6.13) and (6.25)-(6.27) upon the replacement $1 \leftrightarrow 2$,

$$
z_{1} \leftrightarrow z_{2}, \quad \nu_{1} \leftrightarrow \nu_{2}, \quad W_{1,0} \leftrightarrow W_{0,1}, \quad W_{1,1} \leftrightarrow W_{1,1}
$$

Also, using (4.36) and (6.46), we verify that all operators $U$ (6.14)-(6.22) are symmetric upon the replacement of external line indices of continuous-spin massless fields, $1 \leftrightarrow 2$,

$$
\begin{aligned}
& U_{v_{1}, v_{2}} \leftrightarrow U_{v_{1}, v_{2}}, \quad U_{1,2} \leftrightarrow U_{1,2}, \quad U_{\beta} \leftrightarrow U_{\beta}, \quad U_{\zeta} \leftrightarrow U_{\zeta}, \quad U_{B} \leftrightarrow U_{B}, \\
& U_{z 1} \leftrightarrow U_{z 1}, \quad U_{z \nu} \leftrightarrow U_{z \nu}, \quad U_{W} \leftrightarrow U_{W} .
\end{aligned}
$$

vi) We see that vertices (6.5)-(6.11) are singular in the massless limit $m_{3} \rightarrow 0$, i.e., the massless limit of the cubic vertices describing interaction of two continuous-spin massless fields with one arbitrary spin massive field is problematic. In appendix F, by analysing equations for cubic vertices, we demonstrate explicitly that cubic vertices describing interaction of two continuous-spin massless fields with one arbitrary spin massless field are not consistent.

\section{Conclusions}

In this paper, we applied the light-cone gauge formalism to build the parity invariant cubic interaction vertices for continuous-spin massless fields and arbitrary spin massive fields. We considered two types of cubic vertices: vertices describing interaction of one continuousspin massless field with two arbitrary spin massive fields and vertices describing interaction of two continuous-spin massless fields with one arbitrary spin massive field. We found the full lists of such vertices. Also we demonstrated that there are no cubic vertices describing consistent interaction of continuous-spin massless fields with arbitrary spin massless fields. We expect that our results have the following interesting applications and generalizations.

i) In this paper, we studied interaction vertices for fields propagating in flat space and demonstrated that there are no cubic vertices describing consistent interaction of continuous-spin massless fields with arbitrary spin massless fields. Following ideas in ref. [46], we believe then that consistent interaction of continuous-spin fields with arbitrary spin massless fields can be constructed by considering fields propagating in AdS space. Metric-like gauge invariant Lagrangian formulation of continuous-spin free AdS field was developed in refs. [6, 7]. We note however that, in the literature, there are many other interesting approaches which, upon a suitable generalization, could also be helpful for studying an interacting continuous-spin AdS field. For the reader convenience, we briefly review various approaches which could be used for the description of an interacting continuous-spin AdS field. In the framework of framelike approach, interacting higher-spin AdS fields are considered in refs. [47-50], while, in the framework of ambient space metric-like approach, interacting higher-spin AdS fields are considered in refs. [51-56]. ${ }^{11}$ In this respect, recent interesting discussion of

\footnotetext{
${ }^{11}$ In frame-like approach, equations of motion for continuous-spin field were studied in ref. [57].
} 
interacting higher-spin AdS fields may be found in refs. [58, 59]. In the frameworks of world-line particle and twistor-like approaches, higher-spin fields are considered, e.g., in refs. [60] and [61-64]. Interesting approach for analysing interacting conformal higher-spin fields in curved background is discussed in ref. [65]. The light-cone gauge formulation of arbitrary spin free AdS fields was developed in refs. [34, 66, 67]. It would be interesting to extend such formulation to the case of interacting continuousspin fields. Extension of Hamiltonian form of AdS higher-spin fields dynamics [68 $]^{12}$ to the case of continuous-spin field could also be of some interest.

ii) In this paper, we developed the light-cone gauge formulation for the bosonic continuous-spin fields. It would be interesting to extend a light-cone gauge formulation to the case of fermionic continuous-spin fields and apply such formulation for studying vertices that describes interaction of fermionic continuous-spin fields with arbitrary spin massive fields as well as for studying supersymmetric continuous-spin field theories. Continuous-spin supermultiplets are studied refs. [8, 11]. Recent interesting discussion of higher-spin supersymmetric theories may be found in refs. [72-74].

iii) The methods for building $s o(d-1,1)$ Lorentz covariant formulation of field dynamics by using light-cone gauge $s o(d-2)$ covariant formulation [33] are most suitable for studying parity invariant vertices. Moreover such Lorentz covariant formulation turns out to be BRST gauge invariant. Therefore, we think that the parity invariant vertices obtained in this paper, can relatively straightforwardly be cast into BRST gauge invariant form. For example, the full list of the parity invariant light-cone gauge cubic vertices for arbitrary spin massless and massive fields in ref. [31] has straightforwardly been cast into BRST gauge invariant form in ref. [38]. BRST formulation of continuous-spin free field was discussed in ref. [75] and it was noted that such formulation has interesting interrelations with the formulations in terms of the unconstrained higher-spin gauge fields in ref. [76]. Discussion of other interesting formulations in terms of unconstrained gauge fields can be found, e.g., in refs. [77-79]. BRST formulations in terms of traceceless higher-spin gauge fields may be found in refs. [80, 81]. Use of BRST gauge fixed Lagrangian for the computation of partition functions of higher-spin fields is discussed in refs. [82, 83].

iv) In this paper, we studied totally symmetric continuous-spin fields. As is known, string theory spectrum involves mixed-symmetry fields. Therefore from the perspective of studying interrelations between continuous-spin fields and string theory it seems reasonable to extend our study to the case of mixed-symmetry fields. We note, presently, even at the level of free fields, little is known about Lagrangian formulation of a continuous-spin mixed-symmetry fields. On the other hands, many interesting formulations for mixed-symmetry massless and massive higher-spin fields were developed (see, e.g., refs. [84-95]). Extension of such formulations to continuous-spin fields could be also of some interest.

\footnotetext{
${ }^{12}$ Alternative Hamiltonian formulations of AdS higher-spin field dynamics may be found in refs. [69-71].
} 
v) In this paper, we studied cubic vertices for continuous-spin massless fields and arbitrary spin massive fields. Extension of our study to quartic vertices by using methods and approaches in refs. [96-101] might contribute to better understanding of continuous-spin field dynamics.

\section{Acknowledgments}

Author cordially thanks Xavier Bekaert for his kind suggestion to use a light-cone gauge approach for the studying interacting continuous-spin fields. This work was supported by the Russian Science Foundation grant 14-42-00047.

\section{A Notation and conventions}

The vector indices of the $s o(d-2)$ algebra take the values $i, j, k, l=1, \ldots, d-2$. Creation operators $\alpha^{i}, v, \zeta$ and the respective annihilation operators $\bar{\alpha}^{i}, \bar{v}, \bar{\zeta}$ are referred to as oscillators in this paper. Commutation relations, the vacuum $|0\rangle$, and hermitian conjugation rules are fixed by the relations

$$
\begin{aligned}
& {\left[\bar{\alpha}^{i}, \alpha^{j}\right]=\delta^{i j}, \quad[\bar{v}, v]=1, \quad[\bar{\zeta}, \zeta]=1, \quad \bar{\alpha}^{i}|0\rangle=0, \quad \bar{v}|0\rangle=0, \quad \bar{\zeta}|0\rangle=0,} \\
& \alpha^{i \dagger}=\bar{\alpha}^{i}, \quad v^{\dagger}=\bar{v}, \quad \zeta^{\dagger}=\bar{\zeta} .
\end{aligned}
$$

The oscillators $\alpha^{i}, \bar{\alpha}^{i}$ and $v, \bar{v}, \zeta, \bar{\zeta}$, transform under the respective vector and scalar representations of the $s o(d-2)$ algebra. We use the following notation for the scalar product of the oscillators

$$
\alpha^{2} \equiv \alpha^{i} \alpha^{i}, \quad \bar{\alpha}^{2} \equiv \bar{\alpha}^{i} \bar{\alpha}^{i}, \quad N_{\alpha} \equiv \alpha^{i} \bar{\alpha}^{i}, \quad N_{\zeta} \equiv \zeta \bar{\zeta}, \quad N_{v} \equiv v \bar{v}
$$

Throughout this paper we use the following definitions for momentum $\mathbb{P}^{i}$ and quantities $B_{a}, \alpha_{a b}$

$$
\mathbb{P}^{i} \equiv \frac{1}{3} \sum_{a=1,2,3} \check{\beta}_{a} p_{a}^{i}, \quad \check{\beta}_{a} \equiv \beta_{a+1}-\beta_{a+2}, \quad B_{a} \equiv \frac{\alpha_{a}^{i} \mathbb{P}^{i}}{\beta_{a}}, \quad \alpha_{a b} \equiv \alpha_{a}^{i} \alpha_{b}^{i},
$$

where $\beta_{a} \equiv \beta_{a+3}$. Various quantities constructed out of the $B_{a}, \alpha_{a b}$ and derivatives of the $B_{a}, \alpha_{a b}$ are defined as

$$
\begin{aligned}
N_{B_{a}} & =B_{a} \partial_{B_{a}}, \quad N_{\alpha_{a b}}=\alpha_{a b} \partial_{\alpha_{a b}}, & \partial_{B_{a}} & =\partial / \partial B_{a}, \quad \partial_{\alpha_{a b}}=\partial / \partial \alpha_{a b}, \\
N_{a} & =N_{B_{a}}+N_{\alpha_{a a+1}}+N_{\alpha_{a+2 a}}, & \nu_{a} & =N_{\alpha_{a a+1}}+N_{\alpha_{a+2 a}}+\frac{d-4}{2}
\end{aligned}
$$

\section{B Continuous-spin field in helicity basis}

Throughout this paper, we use the $s o(d-2)$ covariant basis of light-cone gauge fields propagating in $R^{d-1,1}$ with arbitrary $d \geq 4$. For $d=4$, light-cone gauge fields can also be considered in a helicity basis. As a helicity basis is popular in many studies we decided, for the reader convenience, to work out light-cone gauge formulation of continuous-spin field 
in such basis. To discuss continuous-spin field in a helicity basis we introduce complex coordinates $x^{R}, x^{L}$ defined by the relations

$$
x^{R} \equiv \frac{1}{\sqrt{2}}\left(x^{1}+\mathrm{i} x^{2}\right), \quad x^{L} \equiv \frac{1}{\sqrt{2}}\left(x^{1}-\mathrm{i} x^{2}\right) .
$$

In the frame of the complex coordinates, a vector of the $s o(2)$ algebra $X^{i}$ is decomposed as $X^{i}=X^{R}, X^{L}$, while a scalar product of $s o(2)$ algebra vectors $X^{i}, Y^{i}$ is represented as $X^{i} Y^{i}=X^{R} Y^{L}+X^{L} Y^{R}$. We decompose the vector oscillators as $\alpha^{i}=\alpha^{R}$, $\alpha^{L}$ and, in place of the $s o(2)$ covariant form of ket-vector $|\phi\rangle$ (2.10), we use a helicity basis ket-vector given by

$$
\begin{aligned}
|\phi\rangle & =\left|\phi_{0}\right\rangle+\left|\phi^{R}\right\rangle+\left|\phi^{L}\right\rangle, \\
\left|\phi_{0}\right\rangle & \equiv \phi_{0}(p)|0\rangle, \quad\left|\phi^{R}\right\rangle=\sum_{n=1}^{\infty} \frac{v^{n} \alpha_{L}^{n}}{n !} \phi_{n}(p)|0\rangle, \quad\left|\phi^{L}\right\rangle=\sum_{n=1}^{\infty} \frac{v^{n} \alpha_{R}^{n}}{n !} \phi_{-n}(p)|0\rangle,
\end{aligned}
$$

$\alpha_{L}=\alpha^{R}, \alpha_{R}=\alpha^{L}$, where $\phi_{0}(p)$ is a scalar field, while $\phi_{ \pm n}(p)$ are fields having helicities $\pm n, n=1,2, \ldots, \infty$. We assume the following hermitian conjugation rules for the fields: $\phi_{0}^{\dagger}(p)=\phi_{0}(-p), \phi_{n}^{\dagger}(p)=\phi_{-n}(-p)$. The oscillators and a vacuum $|0\rangle$ satisfy the relations

$$
\left[\bar{\alpha}^{L}, \alpha^{R}\right]=1, \quad\left[\bar{\alpha}^{R}, \alpha^{L}\right]=1, \quad \bar{\alpha}^{R}|0\rangle=0 \quad \bar{\alpha}^{L}|0\rangle=0 .
$$

In the frame of the complex coordinates, the spin operator $M^{i}$ is decomposed as $M^{i}=$ $M^{R}, M^{L}$, while the $s o(2)$ algebra generator $M^{i j}=-M^{j i}$ is represented as $M^{R L}$. We now note that, in the frame of the complex coordinates, a realization of kinematical generators and dynamical generators $(2.24)-(2.28)$ in terms of differential operators acting on ketvector $|\phi\rangle$ (B.2) is given by

\section{Kinematical generators:}

$$
\begin{aligned}
& P^{R}=p^{R}, \quad P^{L}=p^{L}, \quad P^{+}=\beta, \\
& J^{+R}=\partial_{p^{L}} \beta, \quad J^{+L}=\partial_{p^{R}} \beta, \quad J^{+-}=\partial_{\beta} \beta, \\
& J^{R L}=p^{R} \partial_{p^{R}}-p^{L} \partial_{p^{L}}+M^{R L},
\end{aligned}
$$

\section{Dynamical generators:}

$$
\begin{aligned}
P^{-} & =-\frac{2 p^{R} p^{L}+m^{2}}{2 \beta} \\
J^{-R} & =-\partial_{\beta} p^{R}+\partial_{p^{L}} P^{-}+\frac{1}{\beta} M^{R L} p^{R}+\frac{1}{\beta} M^{R}, \\
J^{-L} & =-\partial_{\beta} p^{L}+\partial_{p^{R}} P^{-}-\frac{1}{\beta} M^{R L} p^{L}+\frac{1}{\beta} M^{L},
\end{aligned}
$$

where we use the notation

$$
\beta \equiv p^{+}, \quad \partial_{\beta} \equiv \partial / \partial \beta, \quad \partial_{p^{R}} \equiv \partial / \partial p^{R}, \quad \partial_{p^{L}} \equiv \partial / \partial p^{L} .
$$


The spin operators $M^{R L}, M^{L}$, and $M^{L}$ appearing in (B.7)-(B.10) are given by

$$
\begin{aligned}
M^{R L} & =N_{R}-N_{L}, \quad N_{R}=\alpha^{R} \bar{\alpha}^{L}, \quad N_{L}=\alpha^{L} \bar{\alpha}^{R}, \\
M^{R} & =g_{L} \bar{\alpha}^{R} \bar{v}-v \alpha^{R} g_{R} \Pi^{R L}, \\
M^{L} & =g_{R} \bar{\alpha}^{L} \bar{v}-v \alpha^{L} g_{L} \Pi^{L R},
\end{aligned}
$$

where quantities $g_{R, L}, \Pi^{R L}$, and $\Pi^{L R}$ are defined by the relations

$$
\begin{aligned}
g_{R} & =\left(\frac{F_{R}}{2\left(N_{R}+1\right)^{2}}\right)^{1 / 2}, & g_{L} & =\left(\frac{F_{L}}{2\left(N_{L}+1\right)^{2}}\right)^{1 / 2}, \\
F_{R} & =\kappa^{2}-N_{R}\left(N_{R}+1\right) m^{2}, & F_{L} & =\kappa^{2}-N_{L}\left(N_{L}+1\right) m^{2}, \\
\Pi^{R L} & =1-\alpha^{L} \frac{1}{N_{L}+1} \bar{\alpha}^{R}, & \Pi^{L R} & =1-\alpha^{R} \frac{1}{N_{R}+1} \bar{\alpha}^{L} .
\end{aligned}
$$

Relations in (B.5)-(B.17) provide the complete description of a realization for the generators of the Poincare algebra $i s o(3,1)$ in terms of differential operators acting on the ket-vector $|\phi\rangle$ (B.2). To our knowledge, the realization of the operators $M^{R}, M^{L}$ for continuous-spin field in $R^{3,1}$ with arbitrary $\kappa^{2}>0$ and $m^{2}<0$ given in (B.13)-(B.17) has not been discussed in earlier literature. ${ }^{13}$

For the reader convenience, we note the following commutators for the spin operators:

$$
\left[M^{R}, M^{L}\right]=-m^{2} M^{R L}, \quad\left[M^{R L}, M^{R}\right]=M^{R}, \quad\left[M^{R L}, M^{L}\right]=-M^{L} .
$$

Action of the operators $\Pi^{R L}, \Pi^{L R}$ (B.17) on various ket-vectors defined in (B.3) is given by

$$
\begin{aligned}
& \Pi^{R L}\left|\phi_{0}\right\rangle=\left|\phi_{0}\right\rangle, \quad \Pi^{R L}\left|\phi^{R}\right\rangle=\left|\phi^{R}\right\rangle, \quad \Pi^{R L}\left|\phi^{L}\right\rangle=0, \\
& \Pi^{L R}\left|\phi_{0}\right\rangle=\left|\phi_{0}\right\rangle, \quad \Pi^{L R}\left|\phi^{R}\right\rangle=0, \quad \Pi^{L R}\left|\phi^{L}\right\rangle=\left|\phi^{L}\right\rangle .
\end{aligned}
$$

We note the following helpful relations for the operators $\Pi^{R L}, \Pi^{L R}$ (B.17) and the oscillators

$$
\begin{aligned}
& \Pi^{R L} \Pi^{R L}=\Pi^{R L}, \quad \Pi^{L R} \Pi^{L R}=\Pi^{L R}, \quad \Pi^{R L} \Pi^{L R}=\Pi^{L R} \Pi^{R L}, \\
& \Pi^{R L} \alpha^{L}=0, \quad \Pi^{L R} \alpha^{R}=0, \quad \bar{\alpha}^{R} \Pi^{R L}=0, \quad \bar{\alpha}^{L} \Pi^{L R}=0 .
\end{aligned}
$$

Hermitian conjugation rules for various quantities above-defined are given by

$$
\begin{aligned}
& \alpha^{R \dagger}=\bar{\alpha}^{L}, \quad \alpha^{L \dagger}=\bar{\alpha}^{R}, \\
& N_{R}^{\dagger}=N_{R}, \quad N_{L}^{\dagger}=N_{L}, \quad \Pi^{R L \dagger}=\Pi^{R L}, \quad \Pi^{L R \dagger}=\Pi^{L R} .
\end{aligned}
$$

To quadratic order in fields, a field representation for generators of the Poincaré algebra takes the form

$$
G_{[2]}=\int \beta d \beta d^{2} p\left\langle\phi(p)\left|G_{[2]}\right| \phi(p)\right\rangle, \quad\langle\phi(p)|\equiv| \phi(p)\rangle^{\dagger},
$$

where $G$ in (B.25) are given in (B.5)-(B.10). The Poisson-Dirac commutator for fields entering ket-vector takes the form

$$
\left.\left[\phi_{k^{\prime}}\left(p^{\prime}\right), \phi_{k^{\prime \prime}}\left(p^{\prime \prime}\right)\right]\right|_{\text {equal } x^{+}}=\frac{1}{2 \beta^{\prime}} \delta\left(\beta^{\prime}+\beta^{\prime \prime}\right) \delta^{2}\left(p^{\prime}+p^{\prime \prime}\right) \delta_{k^{\prime}+k^{\prime \prime}, 0}, \quad k^{\prime}, k^{\prime \prime} \in \mathbb{Z} .
$$

\footnotetext{
${ }^{13}$ For continuous-spin massless field, $m=0$, the operators $M^{R}, M^{L}$ were discussed in section 2 in ref. [11].
} 


\section{Derivation of cubic vertices $p_{[3]}^{-}(4.2),(5.2)$}

Our aim in this appendix is to outline some details of the derivation of cubic vertices given in (4.2), (5.2). We would like to divide our derivation in ten steps which we now discuss in turn.

Step 1. Realization on $\boldsymbol{p}_{[3]}^{-}$. First, we find realization of operators $G_{a, \mathbb{P}^{2}}, G_{\beta}(3.54)$ on vertex $p_{[3]}^{-}$(4.3). To this end we use $\mathbf{J}^{-i \dagger}$ (3.30) with the spin operators $M_{a}^{i}, a=1,2$, for the arbitrary spin massive fields (2.40) and the spin operator $M_{a}^{i}, a=3$, for the continuousspin massless field (2.34)-(2.38). Doing so, we find that action of $\mathbf{J}^{-i \dagger}(3.30)$ on $p_{[3]}^{-}(4.3)$ can be cast into the form (3.54) with the following expressions for the $G_{a, \mathbb{P}^{2}}, G_{\beta}$ :

$$
\begin{aligned}
G_{1, \mathbb{P}^{2}=} & G_{1}, \quad G_{2, \mathbb{P}^{2}}=G_{2}, \quad G_{3, \mathbb{P}^{2}}=G_{3}+\mathbf{P}^{-} \frac{2 \beta}{\beta_{3}^{3}} \alpha_{3}^{i} \frac{g_{v_{3}} \partial_{v_{3}}}{2 N_{3}+d-2} \partial_{B_{3}}^{2}, \\
G_{1}= & \left(B_{3}-\frac{\beta_{1}}{\beta_{3}} g_{v_{3}} \partial_{v_{3}}\right) \partial_{\alpha_{31}}-\left(B_{2}+\frac{\beta_{1}}{\beta_{2}} m_{2} \zeta_{2}\right) \partial_{\alpha_{12}}+\frac{1}{2}\left(\frac{\check{\beta}_{1}}{\beta_{1}} m_{1}^{2}+m_{2}^{2}\right) \partial_{B_{1}}+m_{1} \partial_{\zeta_{1}}, \\
G_{2}= & \left(B_{1}-\frac{\beta_{2}}{\beta_{1}} \zeta_{1} m_{1}\right) \partial_{\alpha_{12}}-\left(B_{3}+\frac{\beta_{2}}{\beta_{3}} g_{v_{3}} \partial_{v_{3}}\right) \partial_{\alpha_{23}}+\frac{1}{2}\left(\frac{\check{\beta}_{2}}{\beta_{2}} m_{2}^{2}-m_{1}^{2}\right) \partial_{B_{2}}+m_{2} \partial_{\zeta_{2}}, \\
G_{3}= & \left(B_{2}-\frac{\beta_{3}}{\beta_{2}} m_{2} \zeta_{2}\right) \partial_{\alpha_{23}}-\left(B_{1}+\frac{\beta_{3}}{\beta_{1}} m_{1} \zeta_{1}\right) \partial_{\alpha_{31}}+\frac{1}{2}\left(m_{1}^{2}-m_{2}^{2}\right) \partial_{B_{3}}+v_{3} g_{v_{3}} \\
& +\frac{g_{v_{3}} \partial_{v_{3}}}{2 N_{3}+d-2}\left(\frac{2 \beta_{1}}{\beta_{3}} B_{1} \partial_{B_{3}} \partial_{\alpha_{31}}+\frac{2 \beta_{2}}{\beta_{3}} B_{2} \partial_{B_{3}} \partial_{\alpha_{23}}\right. \\
& \left.+2 \alpha_{12} \partial_{\alpha_{31}} \partial_{\alpha_{23}}+\alpha_{11} \partial_{\alpha_{31}}^{2}+\alpha_{22} \partial_{\alpha_{23}}^{2}+\frac{\beta}{\beta_{3}^{2}} \sum_{b=1,2} \frac{m_{b}^{2}}{\beta_{b}} \partial_{B_{3}}^{2}\right), \\
G_{\beta}= & -\frac{1}{\beta} \mathbb{N}_{\beta}-\frac{1}{\beta_{1}^{2}} m_{1} \zeta_{1} \partial_{B_{1}}-\frac{1}{\beta_{2}^{2}} m_{2} \zeta_{2} \partial_{B_{2}}-\frac{1}{\beta_{3}^{2}} g_{v_{3}} \partial_{v_{3}} \partial_{B_{3}},
\end{aligned}
$$

where $g_{v}$ and $\check{\beta}_{a}, \mathbb{N}_{\beta}$ are given in (2.37) and (3.34) respectively, while the $B_{a}, \alpha_{a b}, N_{a}$ are given in (A.4)-(A.6). Using (3.40), (3.54), and operators $G_{a, \mathbb{P}^{2}}, G_{\beta}$ (C.1)-(C.5), we see that requiring the density $\left|j_{[3]}^{-i}\right\rangle$ to respect equations (3.43) amounts to the eqs. (3.55), (3.56).

Step 2. Realization on $\boldsymbol{V}^{(\mathbf{1})}$. At this step, we fix dependence of $p_{[3]}^{-}$(4.3) on the oscillator $v_{3}$. To this end, we note that in view of constraint (2.11), vertex $p_{[3]}^{-}$(4.3) should satisfy the constraint

$$
\left(N_{\alpha_{3}}-N_{v_{3}}\right)\left|p_{[3]}^{-}\right\rangle=0 .
$$

Introducing a vertex $V^{(1)}$ by the relations

$$
p_{[3]}^{-}=U_{v_{3}} V^{(1)}, \quad U_{v_{3}}=v_{3}^{N_{3}}, \quad N_{3} \equiv N_{B_{3}}+N_{\alpha_{31}}+N_{\alpha_{23}},
$$

and using (C.6), we find that the $V^{(1)}$ is independent of the oscillator $v_{3}$, i.e., we get

$$
V^{(1)}=V^{(1)}\left(\beta_{a}, B_{a}, \alpha_{a a+1}, \zeta_{1}, \zeta_{2}\right)
$$


Using (C.7), we find that, on vertex $V^{(1)}(\mathrm{C} .8)$, operators $G_{a}, G_{\beta}$ (C.2)-(C.5) are realized as

$$
\begin{aligned}
& G_{1}=\left(B_{3}-\frac{\beta_{1}}{\beta_{3}} g_{3}\left(N_{3}+1\right)\right) \partial_{\alpha_{31}}-\left(B_{2}+\frac{\beta_{1}}{\beta_{2}} m_{2} \zeta_{2}\right) \partial_{\alpha_{12}}+\frac{1}{2}\left(\frac{\check{\beta}_{1}}{\beta_{1}} m_{1}^{2}+m_{2}^{2}\right) \partial_{B_{1}}+m_{1} \partial_{\zeta_{1}} \\
& G_{2}=\left(B_{1}-\frac{\beta_{2}}{\beta_{1}} \zeta_{1} m_{1}\right) \partial_{\alpha_{12}}-\left(B_{3}+\frac{\beta_{2}}{\beta_{3}} g_{3}\left(N_{3}+1\right)\right) \partial_{\alpha_{23}}+\frac{1}{2}\left(\frac{\check{\beta}_{2}}{\beta_{2}} m_{2}^{2}-m_{1}^{2}\right) \partial_{B_{2}}+m_{2} \partial_{\zeta_{2}} \\
& G_{3}=\left(B_{2}-\frac{\beta_{3}}{\beta_{2}} m_{2} \zeta_{2}\right) \partial_{\alpha_{23}}-\left(B_{1}+\frac{\beta_{3}}{\beta_{1}} m_{1} \zeta_{1}\right) \partial_{\alpha_{31}}+\frac{1}{2}\left(m_{1}^{2}-m_{2}^{2}\right) \partial_{B_{3}}+g_{3} \\
&+\frac{g_{3}^{(1)}\left(N_{3}+2\right)}{2 N_{3}+d-2}\left(\frac{2 \beta_{1}}{\beta_{3}} B_{1} \partial_{B_{3}} \partial_{\alpha_{31}}+\frac{2 \beta_{2}}{\beta_{3}} B_{2} \partial_{B_{3}} \partial_{\alpha_{23}}\right. \\
&\left.\quad+2 \alpha_{12} \partial_{\alpha_{31}} \partial_{\alpha_{23}}+\alpha_{11} \partial_{\alpha_{31}}^{2}+\alpha_{22} \partial_{\alpha_{23}}^{2}+\frac{\beta}{\beta_{3}^{3}} \sum_{b=1,2} \frac{m_{b}^{2}}{\beta_{b}} \partial_{B_{3}}^{2}\right) \\
& G_{\beta}=-\frac{1}{\beta} \mathbb{N}_{\beta}-\frac{1}{\beta_{1}^{2}} m_{1} \zeta_{1} \partial_{B_{1}}-\frac{1}{\beta_{2}^{2}} m_{2} \zeta_{2} \partial_{B_{2}}-\frac{1}{\beta_{3}^{2}} g_{3}\left(N_{3}+1\right) \partial_{B_{3}}, \\
& g_{3} \equiv\left.g_{v_{3}}\right|_{N_{v_{3}} \rightarrow N_{3}},\left.\quad g_{3}^{(1)} \equiv g_{v_{3}}\right|_{N_{v_{3}} \rightarrow N_{3}+1},
\end{aligned}
$$

where $N_{v_{3}}=v_{3} \partial_{v_{3}}, N_{3}$ is defined in (C.7), while $g_{v}$ is given by (2.37), (2.38) for $m^{2}=0$.

Step 3. Realization on $\boldsymbol{V}^{(2)}$. We find it convenient to introduce a vertex $V^{(2)}$ by the relations

$$
V^{(1)}=U_{3} V^{(2)}, \quad U_{3}=\left(\frac{2^{N_{3}} \Gamma\left(N_{3}+\frac{d-2}{2}\right)}{\Gamma\left(N_{3}+1\right)}\right)^{1 / 2} .
$$

We note that the vertex $V^{(2)}$ depends on the same variables as vertex $V^{(1)}$ (C.7),

$$
V^{(2)}=V^{(2)}\left(\beta_{a}, B_{a}, \alpha_{a a+1}, \zeta_{1}, \zeta_{2}\right)
$$

On the vertex $V^{(2)}$, realization of $G_{a}, G_{\beta}$ (C.9)-(C.12) takes more convenient form given by

$$
\begin{aligned}
G_{1}= & \left(B_{3}-\frac{\beta_{1}}{\beta_{3}} \kappa_{3}\right) \partial_{\alpha_{31}}-\left(B_{2}+\frac{\beta_{1}}{\beta_{2}} m_{2} \zeta_{2}\right) \partial_{\alpha_{12}}+\frac{1}{2}\left(\frac{\check{\beta}_{1}}{\beta_{1}} m_{1}^{2}+m_{2}^{2}\right) \partial_{B_{1}}+m_{1} \partial_{\zeta_{1}}, \\
G_{2}= & \left(B_{1}-\frac{\beta_{2}}{\beta_{1}} \zeta_{1} m_{1}\right) \partial_{\alpha_{12}}-\left(B_{3}+\frac{\beta_{2}}{\beta_{3}} \kappa_{3}\right) \partial_{\alpha_{23}}+\frac{1}{2}\left(\frac{\check{\beta}_{2}}{\beta_{2}} m_{2}^{2}-m_{1}^{2}\right) \partial_{B_{2}}+m_{2} \partial_{\zeta_{2}}, \\
G_{3}= & \left(B_{2}-\frac{\beta_{3}}{\beta_{2}} m_{2} \zeta_{2}\right) \partial_{\alpha_{23}}-\left(B_{1}+\frac{\beta_{3}}{\beta_{1}} m_{1} \zeta_{1}\right) \partial_{\alpha_{31}}+\frac{1}{2}\left(m_{1}^{2}-m_{2}^{2}\right) \partial_{B_{3}}+\frac{g_{3}^{2}\left(N_{3}+1\right)}{\kappa_{3}} \\
& +\frac{\kappa_{3}}{2 N_{3}+d-2}\left(\frac{2 \beta_{1}}{\beta_{3}} B_{1} \partial_{B_{3}} \partial_{\alpha_{31}}+\frac{2 \beta_{2}}{\beta_{3}} B_{2} \partial_{B_{3}} \partial_{\alpha_{23}}\right. \\
& \left.+2 \alpha_{12} \partial_{\alpha_{31}} \partial_{\alpha_{23}}+\alpha_{11} \partial_{\alpha_{31}}^{2}+\alpha_{22} \partial_{\alpha_{23}}^{2}+\frac{\beta}{\beta_{3}^{2}} \sum \frac{m_{b}^{2}}{\beta_{b}} \partial_{B_{3}}^{2}\right), \\
G_{\beta}= & -\frac{1}{\beta} \mathbb{N}_{\beta}-\frac{1}{\beta_{1}^{2}} m_{1} \zeta_{1} \partial_{B_{1}}-\frac{1}{\beta_{2}^{2}} m_{2} \zeta_{2} \partial_{B_{2}}-\frac{\kappa_{3}}{\beta_{3}^{2}} \partial_{B_{3}} .
\end{aligned}
$$


Namely, as compared with operators (C.9)-(C.12), operators (C.16)-(C.19) do not involve square roots of $N_{3}$ (C.7). Note, it is the quantities $g_{3}, g_{3}^{(1)}$ (C.13) that enter square roots of the $N_{3}$.

Step 4. Realization on $\boldsymbol{V}^{(3)}$. At this step, we fix dependence of $V^{(2)}$ (C.15) on the momenta $\beta_{1}, \beta_{2}, \beta_{3}$. To this end, we use the transformation

$$
V^{(2)}=U_{\beta} V^{(3)}, \quad U_{\beta}=\exp \left(-\frac{\check{\beta}_{1}}{2 \beta_{1}} m_{1} \zeta_{1} \partial_{B_{1}}-\frac{\check{\beta}_{2}}{2 \beta_{2}} m_{2} \zeta_{2} \partial_{B_{2}}-\frac{\check{\beta}_{3}}{2 \beta_{3}} \kappa_{3} \partial_{B_{3}}\right) .
$$

On vertex $V^{(3)}$ (C.20), operators $G_{a}, G_{\beta}$ (C.16)-(C.19) are realized as ${ }^{14}$

$$
\begin{aligned}
G_{1}= & \left(B_{3}+\frac{1}{2} \kappa_{3}\right) \partial_{\alpha_{31}}-\left(B_{2}-\frac{1}{2} m_{2} \zeta_{2}\right) \partial_{\alpha_{12}}+\frac{1}{2} m_{2}^{2} \partial_{B_{1}}+m_{1} \partial_{\zeta_{1}}, \\
G_{2}= & \left(B_{1}+\frac{1}{2} \zeta_{1} m_{1}\right) \partial_{\alpha_{12}}-\left(B_{3}-\frac{1}{2} \kappa_{3}\right) \partial_{\alpha_{23}}-\frac{1}{2} m_{1}^{2} \partial_{B_{2}}+m_{2} \partial_{\zeta_{2}}, \\
G_{3}= & \left(B_{2}+\frac{1}{2} m_{2} \zeta_{2}\right) \partial_{\alpha_{23}}-\left(B_{1}-\frac{1}{2} m_{1} \zeta_{1}\right) \partial_{\alpha_{31}}+\frac{1}{2}\left(m_{1}^{2}-m_{2}^{2}\right) \partial_{B_{3}} \\
& +\frac{\kappa_{3}}{2 N_{3}+d-2}\left(1-\left(B_{1}+\frac{3}{2} m_{1} \zeta_{1}\right) \partial_{B_{3}} \partial_{\alpha_{31}}-\left(B_{2}-\frac{3}{2} m_{2} \zeta_{2}\right) \partial_{B_{3}} \partial_{\alpha_{23}}\right. \\
& \left.+2 \alpha_{12} \partial_{\alpha_{31}} \partial_{\alpha_{23}}+\alpha_{11} \partial_{\alpha_{31}}^{2}+\alpha_{22} \partial_{\alpha_{23}}^{2}-\frac{1}{2}\left(m_{1}^{2}+m_{2}^{2}\right) \partial_{B_{3}}^{2}\right), \\
G_{\beta}= & -\frac{1}{\beta} \mathbb{N}_{\beta} .
\end{aligned}
$$

We find then the following equations for the vertex $V^{(3)}$,

$$
\sum_{a=1,2,3} \beta_{a} \partial_{\beta_{a}} V^{(3)}=0, \quad \mathbb{N}_{\beta} V^{(3)}=0
$$

Namely, the first equation in (C.25) is obtained from (3.60), while the second equation in (C.25) is obtained by using (3.56) and (C.24). Equations (C.25) imply that the vertex $V^{(3)}$ does not dependent of the momenta $\beta_{1}, \beta_{2}, \beta_{3}$. Thus we have the following representation for the vertex $V^{(3)}$ :

$$
V^{(3)}=V^{(3)}\left(B_{a}, \alpha_{a a+1}, \zeta_{1}, \zeta_{2}\right)
$$

Step 5. Realization on $\boldsymbol{V}^{(4)}$. We find it convenient to introduce a vertex $V^{(4)}$ by the relations

$$
V^{(3)}=U_{\zeta} V^{(4)}, \quad U_{\zeta}=\exp \left(-\frac{m_{2}^{2}}{2 m_{1}} \zeta_{1} \partial_{B_{1}}+\frac{m_{1}^{2}}{2 m_{2}} \zeta_{2} \partial_{B_{2}}\right)
$$

We note that the vertex $V^{(4)}$ depends on the same variables as vertex $V^{(3)}$ (C.26),

$$
V^{(4)}=V^{(4)}\left(B_{a}, \alpha_{a a+1}, \zeta_{1}, \zeta_{2}\right) .
$$

\footnotetext{
${ }^{14}$ To analyse equations like $G_{a} V=0$ we find it convenient to use equivalence classes for the operators $G_{a}$. Namely, the operators $G_{a}$ and $\left(2 N_{a}+d-2\right) G_{a}$ are considered to be equivalent.
} 
On the vertex $V^{(4)}$, realization of $G_{a}$ (C.21)-(C.23) takes more convenient form given by

$$
\begin{aligned}
G_{1}= & \left(B_{3}+\frac{1}{2} \kappa_{3}\right) \partial_{\alpha_{31}}-\left(B_{2}-\frac{m_{1}^{2}+m_{2}^{2}}{2 m_{2}} \zeta_{2}\right) \partial_{\alpha_{12}}+m_{1} \partial_{\zeta_{1}} \\
G_{2}= & \left(B_{1}+\frac{m_{1}^{2}+m_{2}^{2}}{2 m_{1}} \zeta_{1}\right) \partial_{\alpha_{12}}-\left(B_{3}-\frac{1}{2} \kappa_{3}\right) \partial_{\alpha_{23}}+m_{2} \partial_{\zeta_{2}} \\
G_{3}= & \left(B_{2}+\frac{m_{2}^{2}-m_{1}^{2}}{2 m_{2}} \zeta_{2}\right) \partial_{\alpha_{23}}-\left(B_{1}+\frac{m_{2}^{2}-m_{1}^{2}}{2 m_{1}} \zeta_{1}\right) \partial_{\alpha_{31}}+\frac{1}{2}\left(m_{1}^{2}-m_{2}^{2}\right) \partial_{B_{3}} \\
& +\frac{\kappa_{3}}{2 N_{3}+d-2}\left(1-\left(B_{1}+\frac{3 m_{1}^{2}+m_{2}^{2}}{2 m_{1}} \zeta_{1}\right) \partial_{B_{3}} \partial_{\alpha_{31}}-\left(B_{2}-\frac{3 m_{2}^{2}+m_{1}^{2}}{2 m_{2}} \zeta_{2}\right) \partial_{B_{3}} \partial_{\alpha_{23}}\right. \\
& \left.\quad+2 \alpha_{12} \partial_{\alpha_{31}} \partial_{\alpha_{23}}+\alpha_{11} \partial_{\alpha_{31}}^{2}+\alpha_{22} \partial_{\alpha_{23}}^{2}-\frac{1}{2}\left(m_{1}^{2}+m_{2}^{2}\right) \partial_{B_{3}}^{2}\right)
\end{aligned}
$$

Namely, as compared with (C.21), (C.22), the $G_{1}, G_{2}$ in (C.29), (C.30) are independent of $\partial_{B_{1}}, \partial_{B_{2}}$.

Step 6. Realization on $\boldsymbol{V}^{(5)}$. At this step, we fix dependence of $V^{(4)}$ (C.28) on the variables $\zeta_{1}, \zeta_{2}$. To this end, we use the transformation

$$
\begin{aligned}
V^{(4)}= & U_{B} V^{(5)} \\
U_{B}= & \exp \left(\left(\frac{\zeta_{1}}{m_{1}} B_{2}-\frac{\zeta_{2}}{m_{2}} B_{1}-\frac{m_{1}^{2}+m_{2}^{2}}{2 m_{1} m_{2}} \zeta_{1} \zeta_{2}\right) \partial_{\alpha_{12}}\right. \\
& \left.\quad-\frac{\zeta_{1}}{m_{1}}\left(B_{3}+\frac{1}{2} \kappa_{3}\right) \partial_{\alpha_{31}}+\frac{\zeta_{2}}{m_{2}}\left(B_{3}-\frac{1}{2} \kappa_{3}\right) \partial_{\alpha_{23}}\right) .
\end{aligned}
$$

On vertex $V^{(5)}$ (C.32), operators $G_{a}, G_{\beta}$ (C.29)-(C.31) are realized as

$$
\begin{aligned}
G_{1}= & m_{1} \partial_{\zeta_{1}}, \quad G_{2}=m_{2} \partial_{\zeta_{2}} \\
G_{3}= & B_{2} \partial_{\alpha_{23}}-B_{1} \partial_{\alpha_{31}}+\frac{1}{2}\left(m_{1}^{2}-m_{2}^{2}\right) \partial_{B_{3}} \\
& +\frac{\kappa_{3}}{2 N_{3}+d-2}\left(1-B_{1} \partial_{B_{3}} \partial_{\alpha_{31}}-B_{2} \partial_{B_{3}} \partial_{\alpha_{23}}\right. \\
& \left.\quad+2 \alpha_{12} \partial_{\alpha_{31}} \partial_{\alpha_{23}}+\left(\alpha_{11}+\zeta_{1}^{2}\right) \partial_{\alpha_{31}}^{2}+\left(\alpha_{22}+\zeta_{2}^{2}\right) \partial_{\alpha_{23}}^{2}-\frac{1}{2}\left(m_{1}^{2}+m_{2}^{2}\right) \partial_{B_{3}}^{2}\right) .
\end{aligned}
$$

Using (C.33) in (3.55) when $a=1,2$, we see that the vertex $V^{(5)}$ is independent of the oscillators $\zeta_{1}, \zeta_{2}$,

$$
V^{(5)}=V^{(5)}\left(B_{a}, \alpha_{a a+1}\right) .
$$

Thus all that remains is to solve the equation

$$
G_{3} V^{(5)}=0
$$

with $G_{3}$ as in (C.34). Up to this point our treatment has been applied on an equal footing to vertices for massive fields having the same masses (4.1) and for massive fields having different masses (5.1). From now on, we separately consider eq. (C.36) for vertices (4.1) and (5.1). 
Step 7. Case $\boldsymbol{m}_{\mathbf{1}}=\boldsymbol{m}_{\mathbf{2}}$. Realization on $\boldsymbol{V}^{(\mathbf{5})}$. We now cast operator $G_{3}$ (C.34) and eq. (C.36) into more convenient form. First, we set $m_{1}=m_{2}=m$ in (C.34). Second, in place of the $B_{3}$, we use variable $z_{3}$ (4.8). Third, we note that in view of constraint (2.17), contribution to commutators (3.25) of $\left(\alpha_{a a}+\zeta_{a}^{2}\right)$-terms, $a=1$, 2, appearing in (C.34) cancel out. Therefore we drop down the just mentioned terms in $G_{3}$ (C.34). Also we multiply eq. (C.36) by the factor $\left(2 N_{3}+d-2\right) / \kappa_{3}$. Doing so, and using notation in (4.8), (4.15)(4.19), we verify that equation for the $V^{(5)}$ takes the form as in (C.36) with the following expression for $G_{3}$ :

$$
G_{3}=1-\partial_{z_{3}}^{2}+X \partial_{z_{3}}+Y\left(N_{z_{3}}+\nu_{3}\right)+Z, \quad N_{z_{3}} \equiv z_{3} \partial_{z_{3}} .
$$

Step 8. Case $\boldsymbol{m}_{\mathbf{1}}=\boldsymbol{m}_{\mathbf{2}}$. Realization on $\boldsymbol{V}^{(\mathbf{6})}$. Operator $G_{3}$ (C.37) is a second-order differential operator with respect to the variable $z_{3}$. We now use the transformation

$$
V^{(5)}=U_{z 2} V^{(6)}, \quad U_{z 2}=\exp \left(\frac{1}{2} z_{3} X+\frac{1}{4} z_{3}^{2} Y\right)
$$

and verify that, on the vertex $V^{(6)}$, operator $G_{3}$ (C.37) is realized as

$$
G_{3}=-\partial_{z_{3}}^{2}+\Omega_{3}^{2}
$$

where $\Omega_{3}^{2}$ is given in (4.18). Remarkable feature of $G_{3}$ (C.39) is that operator $\Omega_{3}^{2}(4.18)$ is independent of the $z_{3}$. Solution to equation for $V^{(6)}(4.31)$ can be presented as in (4.5), (4.6).

Step 7. Case $\boldsymbol{m}_{\mathbf{1}} \neq \boldsymbol{m}_{\mathbf{2}}$. Realization on $\boldsymbol{V}^{(\mathbf{5})}$. Here we cast operator $G_{3}$ (C.34) and eq. (C.36) to more convenient form. First, in place of $B_{3}$, we introduce variable $z_{3}(5.8)$. Second, we note that in view of (2.17), contribution to commutators $(3.25)$ of $\left(\alpha_{a a}+\zeta_{a}^{2}\right)$ terms, $a=1,2$ appearing in (C.34) cancel out. Therefore we drop down the just mentioned terms in $G_{3}$ (C.34). Also we multiply eq. (C.36) by overall factor $\left(2 N_{3}+d-2\right) / \kappa_{3}$. Doing so, and using notation in (5.9) and (5.18)-(5.21) we verify that equation for the $V^{(5)}$ takes the form as in (C.36) with the following $G_{3}$

$$
G_{3}=1-\left(N_{z_{3}}+\nu_{3}+1\right) \partial_{z_{3}}+X \partial_{z_{3}}+\left(N_{z_{3}}+\nu_{3}+1\right) Y+Z .
$$

Step 8. Case $\boldsymbol{m}_{\mathbf{1}} \neq \boldsymbol{m}_{\mathbf{2}}$. Realization on $\boldsymbol{V}^{(\mathbf{6})}$. Operator $G_{3}$ (C.40) is a secondorder differential operator with respect to the variable $z_{3}$. To simplify the $G_{3}$, we use the transformation

$$
V^{(5)}=U_{z 1} V^{(6)}, \quad U_{z 1}=e^{-X+z_{3} Y},
$$

and verify that, on the vertex $V^{(6)}$, operator $G_{3}$ (C.40) is realized as

$$
G_{3}=1-\left(N_{z_{3}}+\nu_{3}+1\right) \partial_{z_{3}}+W, \quad W=Z+X Y
$$

Remarkable feature of $G_{3}$ (C.42) is that operator $W$ (C.42) is independent of the $z_{3}$. Therefore, as we demonstrate below, the equation $G_{3} V^{(6)}=0$ can be solved in terms of the Bessel functions. 
Step 9. Case $\boldsymbol{m}_{\mathbf{1}} \neq \boldsymbol{m}_{\mathbf{2}}$. Realization on $\boldsymbol{V}^{(7)}$. To get more convenient form for $G_{3}$ (C.42), we use the transformation

$$
V^{(6)}=U_{z \nu} V^{(7)}, \quad U_{z \nu}=z_{3}^{-\nu_{3} / 2}
$$

and verify that, on the vertex $V^{(7)}$, operator $G_{3}$ (C.42) is realized as

$$
G_{3}=1-\left(N_{z_{3}}+1\right) \partial_{z_{3}}+\frac{\Omega_{3}^{2}}{4 z_{3}}, \quad \Omega_{3}^{2}=\nu_{3}^{2}+4 W .
$$

Solution to equation $G_{3} V^{(7)}=0$ with $G_{3}$ as in (C.44) can be expressed as

$$
V^{(7)}=I_{\Omega_{3}}\left(\sqrt{4 z_{3}}\right) V^{\prime}, \quad K_{\Omega_{3}}\left(\sqrt{4 z_{3}}\right) V^{\prime}, \quad V^{\prime}=V^{\prime}\left(B_{1}, B_{2}, \alpha_{12}, \alpha_{23}, \alpha_{31}\right) .
$$

where $I_{\Omega_{3}}, K_{\Omega_{3}}$ are the modified Bessel functions. Operator $\nu_{3}$ (5.9) entering $\Omega_{3}^{2}$ (C.44) is diagonal on vertex $V^{\prime}$ (C.45), while the operator $\Omega_{3}$ is not diagonal.

Step 10. Case $m_{1} \neq m_{2}$. Realization on $\boldsymbol{V}^{(8)}$. Our aim is to diagonalize operator $\Omega_{3}$ (C.44). To this end we use the transformation

$$
V^{(7)}=U_{W} V^{(8)} \quad U_{W}=\sum_{m=0}^{\infty} \frac{\Gamma\left(\nu_{3}+m\right)}{m ! \Gamma\left(\nu_{3}+2 m\right)} W^{m} .
$$

Using the relation

$$
\left(\nu_{3}^{2}+4 W\right) U_{W}=U_{W} \nu_{3}^{2},
$$

we see that, on the vertex $V^{(8)}$, operator $\Omega_{3}^{2}$ (C.44) becomes diagonal and operator $G_{3}$ (C.44) takes the form

$$
G_{3}=1-\left(N_{z_{3}}+1\right) \partial_{z_{3}}+\frac{\nu_{3}^{2}}{4 z_{3}} .
$$

Solutions to equation $G_{3} V^{(8)}=0$ with $G_{3}$ as in (C.48) are given in (5.5)-(5.7). Thus we see that the vertex $p_{[3]}^{-}$takes the form given in (5.2)-(5.21).

\section{Derivation of cubic vertex $p_{[3]}^{-}(6.2)$}

In this appendix, we outline some details of the derivation of cubic vertex (6.2). We divide our derivation in ten steps which we now discuss in turn.

Step 1. Realization on $\boldsymbol{p}_{[3]}^{-}$. First, we find realization of operators $G_{a, \mathbb{P}^{2}}, G_{\beta}(3.54)$ on vertex $p_{[3]}^{-}$(6.2). To this end we use $\mathbf{J}^{-i \dagger}$ (3.30) with the spin operators $M_{a}^{i}, a=1,2$, related to the continuous-spin massless fields (2.34)-(2.38), $m=0$, and the spin operator $M_{a}^{i}, a=3$, related to the arbitrary spin massive field (2.40). Doing so, we find that action of $\mathbf{J}^{-i \dagger}$ (3.30) on $p_{[3]}^{-}$(6.2) can be cast into the form (3.54) with the following expressions 
for the $G_{a, \mathbb{P}^{2}}, G_{\beta}$ :

$$
\begin{aligned}
G_{a, \mathbb{P}^{2}}= & G_{a}+\mathbf{P}^{-} \frac{2 \beta}{\beta_{a}^{3}} \alpha_{a}^{i} \frac{g_{v_{a}} \partial_{v_{a}}}{2 N_{a}+d-2} \partial_{B_{a}}^{2}, \quad a=1,2 ; \quad G_{3, \mathbb{P}^{2}}=G_{3}, \\
G_{1}= & \left(B_{3}-\frac{\beta_{1}}{\beta_{3}} m_{3} \zeta_{3}\right) \partial_{\alpha_{31}}-\left(B_{2} \partial_{\alpha_{12}}+\frac{\beta_{1}}{\beta_{2}} g_{v_{2}} \partial_{v_{2}}\right) \partial_{\alpha_{12}}-\frac{1}{2} m_{3}^{2} \partial_{B_{1}}+v_{1} g_{v_{1}} \\
& +\frac{g_{v_{1}} \partial_{v_{1}}}{2 N_{1}+d-2}\left(\frac{2 \beta_{2}}{\beta_{1}} B_{2} \partial_{B_{1}} \partial_{\alpha_{12}}+\frac{2 \beta_{3}}{\beta_{1}} B_{3} \partial_{B_{1}} \partial_{\alpha_{31}}+2 \alpha_{23} \partial_{\alpha_{12}} \partial_{\alpha_{31}}\right. \\
& \left.+\alpha_{33} \partial_{\alpha_{31}}^{2}+\frac{\beta_{2} m_{3}^{2}}{\beta_{1}} \partial_{B_{1}}^{2}\right), \\
G_{2}= & \left(B_{1}-\frac{\beta_{2}}{\beta_{1}} g_{v_{1}} \partial_{v_{1}}\right) \partial_{\alpha_{12}}-\left(B_{3}+\frac{\beta_{2}}{\beta_{3}} m_{3} \zeta_{3}\right) \partial_{\alpha_{23}}+\frac{1}{2} m_{3}^{2} \partial_{B_{2}}+v_{2} g_{v_{2}} \\
& +\frac{g_{v_{2}} \partial_{v_{2}}}{2 N_{2}+d-2}\left(\frac{2 \beta_{3}}{\beta_{2}} B_{3} \partial_{B_{2}} \partial_{\alpha_{23}}+\frac{2 \beta_{1}}{\beta_{2}} B_{1} \partial_{B_{2}} \partial_{\alpha_{12}}+2 \alpha_{31} \partial_{\alpha_{12}} \partial_{\alpha_{23}}\right. \\
& \left.+\alpha_{33} \partial_{\alpha_{23}}^{2}+\frac{\beta_{1} m_{3}^{2}}{\beta_{2}} \partial_{B_{2}}^{2}\right), \\
G_{3}= & \left(B_{2}-\frac{\beta_{3}}{\beta_{2}} g_{v_{2}} \partial_{v_{2}}\right) \partial_{\alpha_{23}}-\left(B_{1}+\frac{\beta_{3}}{\beta_{1}} g_{v_{1}} \partial_{v_{1}}\right) \partial_{\alpha_{31}}+\frac{\check{\beta}_{3}}{2 \beta_{3}} m_{3}^{2} \partial_{B_{3}}+m_{3} \partial_{\zeta_{3}}, \\
G_{\beta}= & -\frac{1}{\beta} \mathbb{N}-\frac{1}{\beta_{1}^{2}} g_{v_{1}} \partial_{v_{1}} \partial_{B_{1}}-\frac{1}{\beta_{2}^{2}} g_{v_{2}} \partial_{v_{2}} \partial_{B_{2}}-\frac{1}{\beta_{3}^{2}} m_{3} \zeta_{3} \partial_{B_{3}},
\end{aligned}
$$

where $g_{v}$ is given in (2.37), (2.38) for $m=0$, while $\check{\beta}_{a}, \mathbb{N}_{\beta}$ are given in (3.34). The $B_{a}$, $\alpha_{a b}, N_{a}$ are defined in (A.4)-(A.6). Using (3.40), (3.54), and explicit form of operators $G_{a, \mathbb{P}^{2}}, G_{\beta}$ (D.1)-(D.5), we see that requiring the density $\left|j_{[3]}^{-i}\right\rangle$ to respect equations (3.43) amounts to the equations (3.55), (3.56).

Step 2. Realization on $\boldsymbol{V}^{(1)}$. Here, we find dependence of $p_{[3]}^{-}(6.3)$ on the oscillators $v_{1}$ and $v_{2}$. To this end, we note that in view of constraint (2.11), vertex $p_{[3]}^{-}$(6.3) should satisfy the constraints

$$
\left(N_{\alpha_{a}}-N_{v_{a}}\right)\left|p_{[3]}^{-}\right\rangle=0, \quad a=1,2 .
$$

Introducing a vertex $V^{(1)}$ by the relations

$$
p_{[3]}^{-}=U_{v_{1}, v_{2}} V^{(1)}, \quad U_{v_{1}, v_{2}}=v_{1}^{N_{1}} v_{2}^{N_{2}}, \quad N_{1}=N_{B_{1}}+N_{\alpha_{12}}+N_{\alpha_{31}}, \quad N_{2}=N_{B_{2}}+N_{\alpha_{12}}+N_{\alpha_{23}},
$$

we verify that eqs. (D.6) imply that the vertex $V^{(1)}$ does not depend on the $v_{1}, v_{2}$, i.e., we get

$$
V^{(1)}=V^{(1)}\left(\beta_{a}, B_{a}, \alpha_{a a+1}, \zeta_{3}\right) .
$$


Using (D.7), we find that, on vertex $V^{(1)}$ (D.8), operators $G_{a}, G_{\beta}$ (D.2)-(D.5) are realized as

$$
\begin{aligned}
G_{1}= & \left(B_{3}-\frac{\beta_{1}}{\beta_{3}} m_{3} \zeta_{3}\right) \partial_{\alpha_{31}}-\left(B_{2}+\frac{\beta_{1}}{\beta_{2}} g_{2}\left(N_{2}+1\right)\right) \partial_{\alpha_{12}}-\frac{1}{2} m_{3}^{2} \partial_{B_{1}}+g_{1} \\
& +\frac{g_{1}^{(1)}\left(N_{1}+2\right)}{2 N_{1}+d-2}\left(\frac{2 \beta_{2}}{\beta_{1}} B_{2} \partial_{B_{1}} \partial_{\alpha_{12}}+\frac{2 \beta_{3}}{\beta_{1}} B_{3} \partial_{B_{1}} \partial_{\alpha_{31}}+2 \alpha_{23} \partial_{\alpha_{12}} \partial_{\alpha_{31}}\right. \\
& \left.+\alpha_{33} \partial_{\alpha_{31}}^{2}+\frac{\beta_{2} m_{3}^{2}}{\beta_{1}} \partial_{B_{1}}^{2}\right) \\
G_{2}= & \left(B_{1}-\frac{\beta_{2}}{\beta_{1}} g_{1}\left(N_{1}+1\right)\right) \partial_{\alpha_{12}}-\left(B_{3}+\frac{\beta_{2}}{\beta_{3}} m_{3} \zeta_{3}\right) \partial_{\alpha_{23}}+\frac{1}{2} m_{3}^{2} \partial_{B_{2}}+g_{2} \\
& +\frac{g_{2}^{(1)}\left(N_{2}+2\right)}{2 N_{2}+d-2}\left(\frac{2 \beta_{3}}{\beta_{2}} B_{3} \partial_{B_{2}} \partial_{\alpha_{23}}+\frac{2 \beta_{1}}{\beta_{2}} B_{1} \partial_{B_{2}} \partial_{\alpha_{12}}+2 \alpha_{31} \partial_{\alpha_{12}} \partial_{\alpha_{23}}\right. \\
& \left.+\alpha_{33} \partial_{\alpha_{23}}^{2}+\frac{\beta_{1} m_{3}^{2}}{\beta_{2}} \partial_{B_{2}}^{2}\right) \\
G_{3}= & \left(B_{2}-\frac{\beta_{3}}{\beta_{2}} g_{2}\left(N_{2}+1\right)\right) \partial_{\alpha_{23}}-\left(B_{1}+\frac{\beta_{3}}{\beta_{1}} g_{1}\left(N_{1}+1\right)\right) \partial_{\alpha_{31}}+\frac{\check{\beta}_{3}}{2 \beta_{3}} m_{3}^{2} \partial_{B_{3}}+m_{3} \partial_{\zeta_{3}} \\
G_{\beta}= & -\frac{1}{\beta} \mathbb{N}-\frac{1}{\beta_{1}^{2}} g_{1}\left(N_{1}+1\right) \partial_{B_{1}}-\frac{1}{\beta_{2}^{2}} g_{2}\left(N_{2}+1\right) \partial_{B_{2}}-\frac{1}{\beta_{3}^{2}} m_{3} \zeta_{3} \partial_{B_{3}}, \\
g_{1} \equiv & \left.g_{v_{1}}\right|_{N_{v_{1}} \rightarrow N_{1}},\left.\quad g_{2} \equiv g_{v_{2}}\right|_{N_{v_{2}} \rightarrow N_{2}},\left.\quad g_{1}^{(1)} \equiv g_{v_{1}}\right|_{N_{v_{1}} \rightarrow N_{1}+1},\left.\quad g_{2}^{(1)} \equiv g_{v_{2}}\right|_{N_{v_{2}} \rightarrow N_{2}+1}
\end{aligned}
$$

Step 3. Realization on $\boldsymbol{V}^{(2)}$. We find it convenient to introduce a vertex $V^{(2)}$ by the relations

$$
\begin{aligned}
& V^{(1)}=U_{1,2} V^{(2)}, \quad U_{1,2}=\left(\frac{2^{N_{1}} \Gamma\left(N_{1}+\frac{d-2}{2}\right)}{\Gamma\left(N_{1}+1\right)} \frac{2^{N_{2}} \Gamma\left(N_{2}+\frac{d-2}{2}\right)}{\Gamma\left(N_{2}+1\right)}\right)^{1 / 2}, \\
& V^{(2)}=V^{(2)}\left(\beta_{a}, B_{a}, \alpha_{a a+1}, \zeta_{3}\right),
\end{aligned}
$$

where, in (D.15), we show that vertex $V^{(2)}$ depends on the same variables as vertex $V^{(1)}$ (D.7). On the vertex $V^{(2)}$, realization of $G_{a}, G_{\beta}$ (D.9)-(D.12) takes more convenient form given by

$$
\begin{aligned}
G_{1}= & \left(B_{3}-\frac{\beta_{1}}{\beta_{3}} m_{3} \zeta_{3}\right) \partial_{\alpha_{31}}-\left(B_{2}+\frac{\beta_{1}}{\beta_{2}} \kappa_{2}\right) \partial_{\alpha_{12}}-\frac{1}{2} m_{3}^{2} \partial_{B_{1}}+\frac{1}{\kappa_{1}} g_{1}^{2}\left(N_{1}+1\right) \\
& +\frac{\kappa_{1}}{2 N_{1}+d-2}\left(\frac{2 \beta_{2}}{\beta_{1}} B_{2} \partial_{B_{1}} \partial_{\alpha_{12}}+\frac{2 \beta_{3}}{\beta_{1}} B_{3} \partial_{B_{1}} \partial_{\alpha_{31}}+2 \alpha_{23} \partial_{\alpha_{12}} \partial_{\alpha_{31}}\right. \\
& \left.+\alpha_{33} \partial_{\alpha_{31}}^{2}+\frac{\beta_{2} m_{3}^{2}}{\beta_{1}} \partial_{B_{1}}^{2}\right), \\
G_{2}= & \left(B_{1}-\frac{\beta_{2}}{\beta_{1}} \kappa_{1}\right) \partial_{\alpha_{12}}-\left(B_{3}+\frac{\beta_{2}}{\beta_{3}} m_{3} \zeta_{3}\right) \partial_{\alpha_{23}}+\frac{1}{2} m_{3}^{2} \partial_{B_{2}}+\frac{1}{\kappa_{2}} g_{2}^{2}\left(N_{2}+1\right) \\
& +\frac{\kappa_{2}}{2 N_{2}+d-2}\left(\frac{2 \beta_{3}}{\beta_{2}} B_{3} \partial_{B_{2}} \partial_{\alpha_{23}}+\frac{2 \beta_{1}}{\beta_{2}} B_{1} \partial_{B_{2}} \partial_{\alpha_{12}}+2 \alpha_{31} \partial_{\alpha_{12}} \partial_{\alpha_{23}}\right. \\
& \left.+\alpha_{33} \partial_{\alpha_{23}}^{2}+\frac{\beta_{1} m_{3}^{2}}{\beta_{2}} \partial_{B_{2}}^{2}\right)
\end{aligned}
$$




$$
\begin{aligned}
G_{3} & =\left(B_{2}-\frac{\beta_{3}}{\beta_{2}} \kappa_{2}\right) \partial_{\alpha_{23}}-\left(B_{1}+\frac{\beta_{3}}{\beta_{1}} \kappa_{1}\right) \partial_{\alpha_{31}}+\frac{\check{\beta}_{3}}{2 \beta_{3}} m_{3}^{2} \partial_{B_{3}}+m_{3} \partial_{\zeta_{3}} \\
G_{\beta} & =-\frac{1}{\beta} \mathbb{N}-\frac{1}{\beta_{1}^{2}} \kappa_{1} \partial_{B_{1}}-\frac{1}{\beta_{2}^{2}} \kappa_{2} \partial_{B_{2}}-\frac{1}{\beta_{3}^{2}} m_{3} \zeta_{3} \partial_{B_{3}} .
\end{aligned}
$$

Namely, as compared with operators (D.9)-(D.12), operators (D.16)-(D.19) do not involve square roots of the operators $N_{1}, N_{2}$ (D.7). Note, it is the quantities $g_{a}, g_{a}^{(1)}, a=1,2$ (D.13) that enter square roots of the $N_{1}, N_{2}$.

Step 4. Realization on $\boldsymbol{V}^{(3)}$. At this step, we fix dependence of $V^{(2)}$ (D.15) on the momenta $\beta_{1}, \beta_{2}, \beta_{3}$. To this end, using notation in (3.17), we introduce the transformation

$$
V^{(2)}=U_{\beta} V^{(3)}, \quad U_{\beta}=\exp \left(-\frac{\check{\beta}_{1}}{2 \beta_{1}} \kappa_{1} \partial_{B_{1}}-\frac{\check{\beta}_{2}}{2 \beta_{2}} \kappa_{2} \partial_{B_{2}}-\frac{\check{\beta}_{3}}{2 \beta_{3}} m_{3} \zeta_{3} \partial_{B_{3}}\right)
$$

On vertex $V^{(3)}(\mathrm{D} .20)$, operators $G_{a}, G_{\beta}$ (D.16)-(D.19) are realized as

$$
\begin{aligned}
G_{1}= & \left(B_{3}+\frac{1}{2} m_{3} \zeta_{3}\right) \partial_{\alpha_{31}}-\left(B_{2}-\frac{1}{2} \kappa_{2}\right) \partial_{\alpha_{12}}-\frac{1}{2} m_{3}^{2} \partial_{B_{1}} \\
& +\frac{\kappa_{1}}{2 N_{1}+d-2}\left(1-\left(B_{2}+\frac{3}{2} \kappa_{2}\right) \partial_{B_{1}} \partial_{\alpha_{12}}-\left(B_{3}-\frac{3}{2} m_{3} \zeta_{3}\right) \partial_{B_{1}} \partial_{\alpha_{31}}+2 \alpha_{23} \partial_{\alpha_{12}} \partial_{\alpha_{31}}\right. \\
& \left.+\alpha_{33} \partial_{\alpha_{31}}^{2}-\frac{1}{2} m_{3}^{2} \partial_{B_{1}}^{2}\right) \\
G_{2}= & \left(B_{1}+\frac{1}{2} \kappa_{1}\right) \partial_{\alpha_{12}}-\left(B_{3}-\frac{1}{2} m_{3} \zeta_{3}\right) \partial_{\alpha_{23}}+\frac{1}{2} m_{3}^{2} \partial_{B_{2}} \\
& +\frac{\kappa_{2}}{2 N_{2}+d-2}\left(1-\left(B_{3}+\frac{3}{2} m_{3} \zeta_{3}\right) \partial_{B_{2}} \partial_{\alpha_{23}}-\left(B_{1}-\frac{3}{2} \kappa_{1}\right) \partial_{B_{2}} \partial_{\alpha_{12}}+2 \alpha_{31} \partial_{\alpha_{12}} \partial_{\alpha_{23}}\right. \\
& \left.+\alpha_{33} \partial_{\alpha_{23}}^{2}-\frac{1}{2} m_{3}^{2} \partial_{B_{2}}^{2}\right) \\
G_{3}= & \left(B_{2}+\frac{1}{2} \kappa_{2}\right) \partial_{\alpha_{23}}-\left(B_{1}-\frac{1}{2} \kappa_{1}\right) \partial_{\alpha_{31}}+m_{3} \partial_{\zeta_{3}} \\
G_{\beta}= & -\frac{1}{\beta} \mathbb{N} .
\end{aligned}
$$

We find then the following equations for the vertex $V^{(3)}$,

$$
\sum_{a=1,2,3} \beta_{a} \partial_{\beta_{a}} V^{(3)}=0, \quad \mathbb{N}_{\beta} V^{(3)}=0
$$

The first equation in (D.25) is obtained from (3.60), while the second equation in (D.25) is obtained by using (3.56) and (D.24). Equations (D.25) imply that the vertex $V^{(3)}$ (D.20) is independent of the momenta $\beta_{1}, \beta_{2}, \beta_{3}$. Thus, we have the following representation for the $V^{(3)}$ :

$$
V^{(3)}=V^{(3)}\left(B_{a}, \alpha_{a a+1}, \zeta_{3}\right)
$$


Step 5. Realization on $\boldsymbol{V}^{(4)}$. At this step, we fix dependence of $V^{(3)}$ (D.26) on the oscillator $\zeta_{3}$. To this end, we use the transformation

$$
V^{(3)}=U_{\zeta} V^{(4)}, \quad U_{\zeta}=\exp \left(\frac{\zeta_{3}}{m_{3}}\left(B_{1}-\frac{1}{2} \kappa_{1}\right) \partial_{\alpha_{31}}-\frac{\zeta_{3}}{m_{3}}\left(B_{2}+\frac{1}{2} \kappa_{2}\right) \partial_{\alpha_{23}}\right) .
$$

On vertex $V^{(4)}$ (D.27), operators $G_{a}, G_{\beta}$ (D.21)-(D.23) are realized as

$$
\begin{aligned}
G_{1}= & B_{3} \partial_{\alpha_{31}}-\left(B_{2}-\frac{1}{2} \kappa_{2}\right) \partial_{\alpha_{12}}-\frac{1}{2} m_{3}^{2} \partial_{B_{1}} \\
& +\frac{\kappa_{1}}{2 N_{1}+d-2}\left(1-\left(B_{2}+\frac{3}{2} \kappa_{2}\right) \partial_{B_{1}} \partial_{\alpha_{12}}-B_{3} \partial_{B_{1}} \partial_{\alpha_{31}}+2 \alpha_{23} \partial_{\alpha_{12}} \partial_{\alpha_{31}}\right. \\
& \left.+\left(\alpha_{33}+\zeta_{3}^{2}\right) \partial_{\alpha_{31}}^{2}-\frac{1}{2} m_{3}^{2} \partial_{B_{1}}^{2}\right) \\
G_{2}= & \left(B_{1}+\frac{1}{2} \kappa_{1}\right) \partial_{\alpha_{12}}-B_{3} \partial_{\alpha_{23}}+\frac{1}{2} m_{3}^{2} \partial_{B_{2}} \\
& +\frac{\kappa_{2}}{2 N_{2}+d-2}\left(1-B_{3} \partial_{B_{2}} \partial_{\alpha_{23}}-\left(B_{1}-\frac{3}{2} \kappa_{1}\right) \partial_{B_{2}} \partial_{\alpha_{12}}+2 \alpha_{31} \partial_{\alpha_{12}} \partial_{\alpha_{23}}\right. \\
& \left.\quad+\left(\alpha_{33}+\zeta_{3}^{2}\right) \partial_{\alpha_{23}}^{2}-\frac{1}{2} m_{3}^{2} \partial_{B_{2}}^{2}\right) \\
G_{3}= & m_{3} \partial_{\zeta_{3}} .
\end{aligned}
$$

Using (D.30) in (3.55) for $a=3$, we see that the vertex $V^{(4)}$ is independent of the oscillator $\zeta_{3}$,

$$
V^{(4)}=V^{(4)}\left(B_{a}, \alpha_{a a+1}\right) .
$$

Thus all that remains is to solve the equations

$$
G_{1} V^{(4)}=0, \quad G_{2} V^{(4)}=0,
$$

where $G_{1}, G_{2}$ take the form given in (D.28), (D.29).

Step 6. Realization on $\boldsymbol{V}^{(4)}$. Here we cast $G_{1}, G_{2}$ (D.28), (D.29), and eq. (D.32) to more convenient form. First, in place of $B_{1}, B_{2}$ (3.53), we introduce variables $z_{1}$, $z_{2}$ (6.10), (6.11). Second, we note that in view of (2.17), contribution of $\left(\alpha_{33}+\zeta_{3}^{2}\right)$ terms (D.28), (D.29) to commutators (3.25) cancel out. Therefore we drop down the just mentioned terms in $G_{1}, G_{2}$ (D.28), (D.29). Also we multiply first and second equations in (D.32) by the respective overall factors $\left(2 N_{1}+d-2\right) / \kappa_{1}$ and $\left(2 N_{2}+d-2\right) / \kappa_{2}$. Doing so, and using notation in (6.10)-(6.13), we verify that equations for $V^{(4)}$ take the form as in (D.32) with the following $G_{1}, G_{2}$ :

$$
\begin{aligned}
G_{1}= & 1-\left(N_{z_{1}}+\nu_{1}+1\right) \partial_{z_{1}}+\frac{2}{\kappa_{1}}\left(N_{z_{1}}+\nu_{1}+1\right) B_{3} \partial_{\alpha_{31}}-\frac{2 \kappa_{1}}{m_{3}^{2}} B_{3} \partial_{z_{1}} \partial_{\alpha_{31}} \\
& +2 \alpha_{23} \partial_{\alpha_{12}} \partial_{\alpha_{31}}+\frac{2 m_{3}^{2}}{\kappa_{1} \kappa_{2}}\left(N_{z_{1}}+\nu_{1}+1\right) z_{2} \partial_{\alpha_{12}}-\frac{2 \kappa_{1} \kappa_{2}}{m_{3}^{2}} \partial_{z_{1}} \partial_{\alpha_{12}}, \\
G_{2}= & 1-\left(N_{z_{2}}+\nu_{2}+1\right) \partial_{z_{2}}-\frac{2}{\kappa_{2}}\left(N_{z_{2}}+\nu_{2}+1\right) B_{3} \partial_{\alpha_{23}}+\frac{2 \kappa_{2}}{m_{3}^{2}} B_{3} \partial_{z_{2}} \partial_{\alpha_{23}} \\
& +2 \alpha_{31} \partial_{\alpha_{12}} \partial_{\alpha_{23}}+\frac{2 m_{3}^{2}}{\kappa_{1} \kappa_{2}}\left(N_{z_{2}}+\nu_{2}+1\right) z_{1} \partial_{\alpha_{12}}-\frac{2 \kappa_{1} \kappa_{2}}{m_{3}^{2}} \partial_{z_{2}} \partial_{\alpha_{12}},
\end{aligned}
$$


where $N_{z_{a}}=z_{a} \partial_{z_{a}}$. The $G_{1}, G_{2}$ (D.33), (D.34) are 2nd-order differential operators w.r.t. $z_{1}, z_{2}$. Our next aim is to simplify operators $G_{1}, G_{2}$ (D.33), (D.34).

Step 7. Realization on $\boldsymbol{V}^{(5)}$. To simplify $G_{1}, G_{2}$, (D.33), (D.34), we use the transformation

$$
V^{(4)}=U_{z 0} V^{(5)}, \quad U_{z 0}=\exp \left(\frac{2 \kappa_{1} \kappa_{2}}{m_{3}^{2}} \partial_{\alpha_{12}}+\frac{2 \kappa_{1}}{m_{3}^{2}} B_{3} \partial_{\alpha_{31}}-\frac{2 \kappa_{2}}{m_{3}^{2}} B_{3} \partial_{\alpha_{23}}\right)
$$

and verify that, on the vertex $V^{(5)}$, operators $G_{1}, G_{2}$ (D.33), (D.34) are realized as

$$
\begin{aligned}
G_{1}= & 1-\left(N_{z_{1}}+\nu_{1}+1\right) \partial_{z_{1}}+\frac{2}{\kappa_{1}}\left(N_{z_{1}}+\nu_{1}+1\right) B_{3} \partial_{\alpha_{31}}+2 \alpha_{23} \partial_{\alpha_{12}} \partial_{\alpha_{31}} \\
& +\frac{2 m_{3}^{2}}{\kappa_{1} \kappa_{2}}\left(N_{z_{1}}+\nu_{1}+1\right) z_{2} \partial_{\alpha_{12}}-4 z_{2} \partial_{\alpha_{12}}^{2}-\frac{4}{\kappa_{2}} z_{2} B_{3} \partial_{\alpha_{12}} \partial_{\alpha_{31}}-\frac{4}{m_{3}^{2}} B_{3}^{2} \partial_{\alpha_{31}}^{2}, \\
G_{2}= & 1-\left(N_{z_{2}}+\nu_{2}+1\right) \partial_{z_{2}}+\frac{2}{\kappa_{2}}\left(N_{z_{2}}+\nu_{2}+1\right) B_{3} \partial_{\alpha_{23}}+2 \alpha_{31} \partial_{\alpha_{12}} \partial_{\alpha_{23}} \\
& +\frac{2 m_{3}^{2}}{\kappa_{1} \kappa_{2}}\left(N_{z_{2}}+\nu_{2}+1\right) z_{1} \partial_{\alpha_{12}}-4 z_{1} \partial_{\alpha_{12}}^{2}-\frac{4}{\kappa_{1}} z_{1} B_{3} \partial_{\alpha_{12}} \partial_{\alpha_{23}}-\frac{4}{m_{3}^{2}} B_{3}^{2} \partial_{\alpha_{23}}^{2}
\end{aligned}
$$

As compared with (D.33), (D.34), operators $G_{1}, G_{2}$ in (D.36), (D.37) do not involve $\partial_{z_{a}} \partial_{\alpha_{12}}{ }^{-}$ terms and $B_{3} \partial_{z_{a}} \partial_{\alpha_{a 3}}$-terms, $a=1,2$.

Step 8. Realization on $\boldsymbol{V}^{(6)}$. To get more simple form for $G_{1}, G_{2}$ (D.36), (D.37), we use the transformation

$$
V^{(5)}=U_{z 2} V^{(6)}, \quad U_{z 2}=\exp \left(\frac{2 m_{3}^{2}}{\kappa_{1} \kappa_{2}} z_{1} z_{2} \partial_{\alpha_{12}}+\frac{2}{\kappa_{1}} z_{1} B_{3} \partial_{\alpha_{31}}-\frac{2}{\kappa_{2}} z_{2} B_{3} \partial_{\alpha_{23}}\right),
$$

and verify that, on the vertex $V^{(6)}$, operators $G_{1}, G_{2}$ (D.36), (D.37) are realized as

$$
\begin{aligned}
& G_{1}=1-\left(N_{z_{1}}+\nu_{1}+1\right) \partial_{z_{1}}+2 \alpha_{23} \partial_{\alpha_{12}} \partial_{\alpha_{31}}-4 z_{2} \partial_{\alpha_{12}}^{2}-\frac{4}{m_{3}^{2}} B_{3}^{2} \partial_{\alpha_{31}}^{2}, \\
& G_{2}=1-\left(N_{z_{2}}+\nu_{2}+1\right) \partial_{z_{2}}+2 \alpha_{31} \partial_{\alpha_{12}} \partial_{\alpha_{23}}-4 z_{1} \partial_{\alpha_{12}}^{2}-\frac{4}{m_{3}^{2}} B_{3}^{2} \partial_{\alpha_{23}}^{2} .
\end{aligned}
$$

As compared with (D.36), (D.37), operators (D.39), (D.40) do not involve $N_{z_{a}} \partial_{\alpha_{12}}$-terms, $a=1,2$.

Step 9. Realization on $\boldsymbol{V}^{(7)}$. In order to remove the dependence of the $G_{1}$ on the variable $z_{2}$ (D.39) and the dependence of the $G_{2}$ on the variable $z_{1}$ (D.40) we use the transformation

$$
V^{(6)}=U_{z \nu} V^{(7)}, \quad U_{z \nu}=z_{1}^{-\nu_{1} / 2} z_{2}^{-\nu_{2} / 2}
$$

and verify that, on the vertex $V^{(7)}$, operators $G_{1}, G_{2}$ (D.39), (D.40) are realized as

$$
\begin{array}{ll}
G_{1}=1-\left(N_{z_{1}}+1\right) \partial_{z_{1}}+\frac{\Omega_{1}^{2}}{4 z_{1}}, & \Omega_{1}^{2}=\nu_{1}^{2}+4 W_{1,0}+4 W_{1,1}, \\
G_{2}=1-\left(N_{z_{2}}+1\right) \partial_{z_{2}}+\frac{\Omega_{2}^{2}}{4 z_{2}}, & \Omega_{2}^{2}=\nu_{2}^{2}+4 W_{0,1}+4 W_{1,1},
\end{array}
$$

where $W_{1,0}, W_{0,1}, W_{1,1}$ are defined in (6.25)-(6.27). 
Step 10. Realization on $\boldsymbol{V}^{(8)}$. Using commutators (6.33)-(6.39), we verify that $\Omega_{1}^{2}$, $\Omega_{2}^{2}$ (D.42), (D.43) are commuting, $\left[\Omega_{1}^{2}, \Omega_{2}^{2}\right]=0$. We then find a transformation that casts the $\Omega_{1}^{2}, \Omega_{2}^{2}$ into a diagonal form. Namely, we introduce the transformation

$$
V^{(7)}=U_{W} V^{(8)}
$$

where operator $U_{W}$ is defined in (6.22)-(6.27). Using the relations

$$
\Omega_{1}^{2} U_{W}=U_{W} \nu_{1}^{2}, \quad \Omega_{2}^{2} U_{W}=U_{W} \nu_{2}^{2}
$$

we see that, on the vertex $V^{(8)}$, operators $\Omega_{1}^{2}, \Omega_{2}^{2}$ (D.42), (D.43) become diagonal, i.e., on the vertex $V^{(8)}$, operators $G_{1}, G_{2}$ (D.42), (D.43) are realized as

$$
G_{1}=1-\left(N_{z_{1}}+1\right) \partial_{z_{1}}+\frac{\nu_{1}^{2}}{4 z_{1}}, \quad G_{2}=1-\left(N_{z_{2}}+1\right) \partial_{z_{2}}+\frac{\nu_{2}^{2}}{4 z_{2}} .
$$

Solutions to equations $G_{1} V^{(8)}=0, G_{2} V^{(8)}=0$ with $G_{1}, G_{2}$ as in (D.46), are given in (6.5)(6.8). Thus, we see that the vertex $p_{[3]}^{-}$takes the form given in (6.2)-(6.27).

\section{E Analysis of equations to vertices for one continuous-spin massless field and two massless fields}

In this appendix, we prove that one continuous-spin massless field and two arbitrary spin massless fields have no consistent cubic interaction vertices. We divide our proof into the five steps.

Step 1. Realization on $\boldsymbol{p}_{[3]}^{-}$. Using the shortcut $(0, \kappa)_{\mathrm{CSF}}$ for continuous-spin massless field and the shortcut $(0, s)$ for spin- $s$ massless field we consider the cubic vertex for the following three fields:

$$
\left(0, s_{1}\right)-\left(0, s_{2}\right)-\left(0, \kappa_{3}\right)_{\mathrm{CSF}} \quad \text { one continuous-spin massless field and two massless fields. }
$$

Our notation in (E.1) implies that spin- $s_{1}$ and spin- $s_{2}$ massless fields carry external line indices $a=1,2$, while the continuous-spin massless field corresponds to $a=3$. Using notation in (3.53), we note that a general form of parity invariant vertex that respect $J^{i j}$-symmetries is given by

$$
p_{[3]}^{-}=p_{[3]}^{-}\left(\beta_{a}, B_{a}, \alpha_{a a+1}, v_{3}\right) .
$$

To find realization of $G_{a, \mathbb{P}^{2}}, G_{\beta}(3.54)$ on $p_{[3]}^{-}$(E.2) we use $\mathbf{J}^{-i \dagger}$ (3.30), where operators $M_{a}^{i}, a=1,2$, for arbitrary spin massless fields are equal to zero, $(2.41)$, while operator $M_{a}^{i}$, $a=3$, for continuous-spin massless field can be read from (2.34)-(2.38) for $m^{2}=0$. Doing so, we find that action of $\mathbf{J}^{-i \dagger}$ (3.30) on $p_{[3]}^{-}$(E.2) can be cast into the form (3.54) with the 
following $G_{a, \mathbb{P}^{2}}, G_{\beta}$ :

$$
\begin{aligned}
G_{1, \mathbb{P}^{2}}= & G_{1}, \quad G_{2, \mathbb{P}^{2}}=G_{2}, \quad G_{3, \mathbb{P}^{2}}=G_{3}+\mathbf{P}^{-} \frac{2 \beta}{\beta_{3}^{3}} \alpha_{3}^{i} \frac{g_{v_{3}} \partial_{v_{3}}}{2 N_{3}+d-2} \partial_{B_{3}}^{2}, \\
G_{1}= & \left(B_{3}-\frac{\beta_{1}}{\beta_{3}} g_{v_{3}} \partial_{v_{3}}\right) \partial_{\alpha_{31}}-B_{2} \partial_{\alpha_{12}}, \\
G_{2}= & B_{1} \partial_{\alpha_{12}}-\left(B_{3}+\frac{\beta_{2}}{\beta_{3}} g_{v_{3}} \partial_{v_{3}}\right) \partial_{\alpha_{23}}, \\
G_{3}= & B_{2} \partial_{\alpha_{23}}-B_{1} \partial_{\alpha_{31}}+v_{3} g_{v_{3}} \\
& +\frac{g_{v_{3}} \partial_{v_{3}}}{2 N_{3}+d-2}\left(\frac{2 \beta_{1}}{\beta_{3}} B_{1} \partial_{B_{3}} \partial_{\alpha_{31}}+\frac{2 \beta_{2}}{\beta_{3}} B_{2} \partial_{B_{3}} \partial_{\alpha_{23}}+2 \alpha_{12} \partial_{\alpha_{31}} \partial_{\alpha_{23}}\right), \\
G_{\beta}= & -\frac{1}{\beta} \mathbb{N}_{\beta}-\frac{1}{\beta_{3}^{2}} g_{v_{3}} \partial_{v_{3}} \partial_{B_{3}},
\end{aligned}
$$

where $g_{v}$ is given in (2.37), (2.38) for $m=0$, while $\check{\beta}_{a}, \mathbb{N}_{\beta}$ are given in (3.34). The $B_{a}$, $\alpha_{a b}, N_{a}$ are defined in (A.4)-(A.6). Using (3.40), (3.54), and operators $G_{a, \mathbb{P}^{2}}, G_{\beta}$ (E.3)(E.7), we see that requiring the density $\left|j_{[3]}^{-i}\right\rangle$ to respect equations (3.43) amounts to the eqs. (3.55), (3.56).

Step 2. Realization on $\boldsymbol{V}^{(1)}$. At this step, we fix dependence of $p_{[3]}^{-}$(E.2) on the oscillator $v_{3}$. To this end, we note that in view of constraint (2.11), vertex $p_{[3]}^{-}$(E.2) should satisfy the constraint

$$
\left(N_{\alpha_{3}}-N_{v_{3}}\right)\left|p_{[3]}^{-}\right\rangle=0 .
$$

Introducing a vertex $V^{(1)}$ by the relations

$$
p_{[3]}^{-}=U_{v_{3}} V^{(1)}, \quad U_{v_{3}}=v_{3}^{N_{3}}, \quad N_{3} \equiv N_{B_{3}}+N_{\alpha_{31}}+N_{\alpha_{23}},
$$

we obtain from eq. (E.8) that the vertex $V^{(1)}$ is independent of the oscillator $v_{3}$, i.e., we get

$$
V^{(1)}=V^{(1)}\left(\beta_{a}, B_{a}, \alpha_{a a+1}\right) .
$$

Using (E.9), we find that, on vertex $V^{(1)}$ (E.10), operators $G_{a}, G_{\beta}($ E.4)-(E.7) are realized as

$$
\begin{aligned}
G_{1}= & \left(B_{3}-\frac{\beta_{1}}{\beta_{3}} g_{3}\left(N_{3}+1\right)\right) \partial_{\alpha_{31}}-B_{2} \partial_{\alpha_{12}} \\
G_{2}= & B_{1} \partial_{\alpha_{12}}-\left(B_{3}+\frac{\beta_{2}}{\beta_{3}} g_{3}\left(N_{3}+1\right)\right) \partial_{\alpha_{23}} \\
G_{3}= & B_{2} \partial_{\alpha_{23}}-B_{1} \partial_{\alpha_{31}}+g_{3} \\
& +\frac{g_{3}^{(1)}\left(N_{3}+2\right)}{2 N_{3}+d-2}\left(\frac{2 \beta_{1}}{\beta_{3}} B_{1} \partial_{B_{3}} \partial_{\alpha_{31}}+\frac{2 \beta_{2}}{\beta_{3}} B_{2} \partial_{B_{3}} \partial_{\alpha_{23}}+2 \alpha_{12} \partial_{\alpha_{31}} \partial_{\alpha_{23}}\right) \\
G_{\beta}= & -\frac{1}{\beta} \mathbb{N}_{\beta}-\frac{1}{\beta_{3}^{2}} g_{3}\left(N_{3}+1\right) \partial_{B_{3}} \\
g_{3} \equiv & \left.g_{v_{3}}\right|_{N_{v_{3}} \rightarrow N_{3}},\left.\quad g_{3}^{(1)} \equiv g_{v_{3}}\right|_{N_{v_{3}} \rightarrow N_{3}+1}
\end{aligned}
$$

where $N_{v_{3}}=v_{3} \partial_{v_{3}}, N_{3}$ is defined in (E.9), while $g_{v}$ is given by $(2.37),(2.38)$ for $m^{2}=0$. 
Step 3. Realization on $\boldsymbol{V}^{(2)}$. We find it convenient to introduce a vertex $V^{(2)}$ by the relations

$$
V^{(1)}=U_{3} V^{(2)}, \quad V^{(2)}=V^{(2)}\left(\beta_{a}, B_{a}, \alpha_{a a+1}\right), \quad U_{3}=\left(\frac{2^{N_{3}} \Gamma\left(N_{3}+\frac{d-2}{2}\right)}{\Gamma\left(N_{3}+1\right)}\right)^{1 / 2} .
$$

Note that vertex $V^{(2)}$ (E.16) depends on the same variables as vertex $V^{(1)}$ (E.10). On vertex $V^{(2)}$ (E.16), realization of $G_{a}, G_{\beta}$ (E.11)-(E.14) takes more convenient form given by

$$
\begin{aligned}
G_{1}= & \left(B_{3}-\frac{\beta_{1}}{\beta_{3}} \kappa_{3}\right) \partial_{\alpha_{31}}-B_{2} \partial_{\alpha_{12}}, \\
G_{2}= & B_{1} \partial_{\alpha_{12}}-\left(B_{3}+\frac{\beta_{2}}{\beta_{3}} \kappa_{3}\right) \partial_{\alpha_{23}}, \\
G_{3}= & B_{2} \partial_{\alpha_{23}}-B_{1} \partial_{\alpha_{31}}+\frac{g_{3}^{2}\left(N_{3}+1\right)}{\kappa_{3}} \\
& +\frac{\kappa_{3}}{2 N_{3}+d-2}\left(\frac{2 \beta_{1}}{\beta_{3}} B_{1} \partial_{B_{3}} \partial_{\alpha_{31}}+\frac{2 \beta_{2}}{\beta_{3}} B_{2} \partial_{B_{3}} \partial_{\alpha_{23}}+2 \alpha_{12} \partial_{\alpha_{31}} \partial_{\alpha_{23}}\right), \\
G_{\beta}= & -\frac{1}{\beta} \mathbb{N}_{\beta}-\frac{\kappa_{3}}{\beta_{3}^{2}} \partial_{B_{3}} .
\end{aligned}
$$

Namely, as compared with operators (E.11)-(E.14), operators (E.17)-(E.20) do not involve square roots of $N_{3}$ (E.9). Note, it is the quantities $g_{3}, g_{3}^{(1)}$ (E.15) that enter square roots of the $N_{3}$.

Step 4. Realization on $\boldsymbol{V}^{(3)}$. At this step, we remove the dependence of operators $G_{a}$ (E.17)-(E.19) on the momenta $\beta_{1}, \beta_{2}, \beta_{3}$. To this end we use the transformation

$$
V^{(2)}=U_{\beta} V^{(3)}, \quad V^{(3)}=V^{(3)}\left(\beta_{a}, B_{a}, \alpha_{a a+1}\right), \quad U_{\beta}=\exp \left(-\frac{\check{\beta}_{3}}{2 \beta_{3}} \kappa_{3} \partial_{B_{3}}\right) .
$$

On vertex $V^{(3)}$ (E.21), operators $G_{a}, G_{\beta}$ (E.17)-(E.20) are realized as

$$
\begin{aligned}
G_{1} & =\left(B_{3}+\frac{1}{2} \kappa_{3}\right) \partial_{\alpha_{31}}-B_{2} \partial_{\alpha_{12}}, \\
G_{2} & =B_{1} \partial_{\alpha_{12}}-\left(B_{3}-\frac{1}{2} \kappa_{3}\right) \partial_{\alpha_{23}}, \\
G_{3} & =B_{2} \partial_{\alpha_{23}}-B_{1} \partial_{\alpha_{31}}+\frac{\kappa_{3}}{2 N_{3}+d-2}\left(1-B_{1} \partial_{B_{3}} \partial_{\alpha_{31}}-B_{2} \partial_{B_{3}} \partial_{\alpha_{23}}+2 \alpha_{12} \partial_{\alpha_{31}} \partial_{\alpha_{23}}\right), \\
G_{\beta} & =-\frac{1}{\beta} \mathbb{N}_{\beta} .
\end{aligned}
$$

Step 5. Realization on $\boldsymbol{V}^{(4)}$. Solution to equations for vertex $V^{(3)}$ (E.21)

$$
G_{1} V^{(3)}=0, \quad G_{2} V^{(3)}=0,
$$

with $G_{1}, G_{2}$ as in (E.22), (E.23) is given by

$$
\begin{aligned}
V^{(3)} & =V^{(4)}\left(B_{a}, Z\right), \\
Z & =B_{3}\left(B_{1} \alpha_{23}+B_{2} \alpha_{31}+B_{3} \alpha_{12}\right)+\frac{1}{2} \kappa_{3}\left(B_{1} \alpha_{23}-B_{2} \alpha_{31}\right)-\frac{1}{4} \kappa_{3}^{2} \alpha_{12} .
\end{aligned}
$$


Multiplying (E.24) by the factor $\left(2 N_{3}+d-2\right) / \kappa_{3}$, we verify that equation $G_{3} V^{(3)}=0$ with $G_{3}$ and $V^{(3)}$ as in (E.24), (E.27) amounts to the following equation for $V^{(4)}$ (E.27):

$$
\left(B_{1} B_{2}\left(2 N_{Z}+d-4\right) \partial_{Z}+1\right) V^{(4)}=0 .
$$

Solutions to eq. (E.29) are given by

$$
\begin{aligned}
V^{(4)} & =W^{\frac{6-d}{2}} I_{\frac{d-6}{2}}(\sqrt{4 W}) V^{(5)}, & V^{(4)} & =W^{\frac{6-d}{2}} K_{\frac{d-6}{2}}(\sqrt{4 W}) V^{(5)}, \\
V^{(5)} & =V^{(5)}\left(B_{a}\right), & W & =-\frac{Z}{2 B_{1} B_{2}} .
\end{aligned}
$$

Taking into account expression for $Z$ (E.28), we see that a power series expansion of vertices $V^{(4)}$ (E.30) in the $B_{1}, B_{2}$ involves negative powers of the $B_{1}, B_{2}$. Therefore vertices (E.30) are not consistent. To summarize, in the flat space, there are no cubic vertices describing consistent interaction of two arbitrary spin massless fields with one continuous-spin massless field.

\section{F Analysis of equations to vertices for two continuous-spin massless fields and one massless field}

In this appendix, we prove that two continuous-spin massless fields and one arbitrary spin massless field have no consistent cubic interaction vertices. We divide our proof into five steps.

Step 1. Realization on $\boldsymbol{p}_{[3]}^{-}$. Using the shortcut $(0, \kappa)_{\mathrm{CSF}}$ for continuous-spin massless field and the shortcut $(0, s)$ for spin- $s$ massless field we consider a cubic vertex for the following fields

$$
\left(0, \kappa_{1}\right)_{\mathrm{CSF}^{-}}\left(0, \kappa_{2}\right)_{\mathrm{CSF}^{-}}\left(0, s_{3}\right) \quad \text { two continuous-spin massless fields and one massless field. }
$$

Our notation in (F.1) implies that continuous-spin massless fields carry external line indices $a=1,2$, while the spin- $s_{3}$ massless field corresponds to $a=3$. Using notation in (3.53), we note that a general form of parity invariant vertex that respect $J^{i j}$-symmetries is given by

$$
p_{[3]}^{-}=p_{[3]}^{-}\left(\beta_{a}, B_{a}, \alpha_{a a+1}, v_{1}, v_{2}\right) .
$$

To find realization of $G_{a, \mathbb{P}^{2}}, G_{\beta}(3.54)$ on $p_{[3]}^{-}$(F.2) we use $\mathbf{J}^{-i \dagger}$ (3.30), where operators $M_{a}^{i}, a=1,2$ for continuous-spin massless fields can be read from (2.34)-(2.38) for $m^{2}=0$, while operator $M_{a}^{i}, a=3$, for arbitrary spin massless field is equal to zero (2.41). Doing so, we find that action of $\mathbf{J}^{-i \dagger}$ (3.30) on $p_{[3]}^{-}$(F.2) can be cast into the form (3.54) with the 
following $G_{a, \mathbb{P}^{2}}, G_{\beta}$ :

$$
\begin{aligned}
G_{a, \mathbb{P}^{2}}= & G_{a}+\mathbf{P}^{-} \frac{2 \beta}{\beta_{a}^{3}} \alpha_{a}^{i} \frac{g_{v_{a}} \partial_{v_{a}}}{2 N_{a}+d-2} \partial_{B_{a}}^{2}, \quad a=1,2 ; \quad G_{3, \mathbb{P}^{2}}=G_{3}, \\
G_{1}= & B_{3} \partial_{\alpha_{31}}-\left(B_{2} \partial_{\alpha_{12}}+\frac{\beta_{1}}{\beta_{2}} g_{v_{2}} \partial_{v_{2}}\right) \partial_{\alpha_{12}}+v_{1} g_{v_{1}} \\
& +\frac{g_{v_{1}} \partial_{v_{1}}}{2 N_{1}+d-2}\left(\frac{2 \beta_{2}}{\beta_{1}} B_{2} \partial_{B_{1}} \partial_{\alpha_{12}}+\frac{2 \beta_{3}}{\beta_{1}} B_{3} \partial_{B_{1}} \partial_{\alpha_{31}}+2 \alpha_{23} \partial_{\alpha_{12}} \partial_{\alpha_{31}}\right), \\
G_{2}= & \left(B_{1}-\frac{\beta_{2}}{\beta_{1}} g_{v_{1}} \partial_{v_{1}}\right) \partial_{\alpha_{12}}-B_{3} \partial_{\alpha_{23}}+v_{2} g_{v_{2}} \\
& +\frac{g_{v_{2}} \partial_{v_{2}}}{2 N_{2}+d-2}\left(\frac{2 \beta_{3}}{\beta_{2}} B_{3} \partial_{B_{2}} \partial_{\alpha_{23}}+\frac{2 \beta_{1}}{\beta_{2}} B_{1} \partial_{B_{2}} \partial_{\alpha_{12}}+2 \alpha_{31} \partial_{\alpha_{12}} \partial_{\alpha_{23}}\right), \\
G_{3}= & \left(B_{2}-\frac{\beta_{3}}{\beta_{2}} g_{v_{2}} \partial_{v_{2}}\right) \partial_{\alpha_{23}}-\left(B_{1}+\frac{\beta_{3}}{\beta_{1}} g_{v_{1}} \partial_{v_{1}}\right) \partial_{\alpha_{31}}, \\
G_{\beta}= & -\frac{1}{\beta} \mathbb{N}-\frac{1}{\beta_{1}^{2}} g_{v_{1}} \partial_{v_{1}} \partial_{B_{1}}-\frac{1}{\beta_{2}^{2}} g_{v_{2}} \partial_{v_{2}} \partial_{B_{2}},
\end{aligned}
$$

where $g_{v}$ is given in (2.37), (2.38) for $m=0$, while $\check{\beta}_{a}, \mathbb{N}_{\beta}$ are given in (3.34). The $B_{a}, \alpha_{a b}$, $N_{a}$ are defined in (A.4)-(A.6). Using operators $G_{a, \mathbb{P}^{2}}, G_{\beta}$ (F.3)-(F.7) in (3.40), (3.54), we see that requiring the density $\left|j_{[3]}^{-i}\right\rangle$ to respect equations (3.43) amounts to equations (3.55), (3.56).

Step 2. Realization on $\boldsymbol{V}^{(1)}$. We find dependence of the vertex $p_{[3]}^{-}$on the oscillators $v_{1}$ and $v_{2}$. To this end, we note that in view of constraint (2.11), vertex $p_{[3]}^{-}$(4.3) should satisfy the constraints

$$
\left(N_{\alpha_{a}}-N_{v_{a}}\right)\left|p_{[3]}^{-}\right\rangle=0, \quad a=1,2 .
$$

Introducing a vertex $V^{(1)}$ by the relations

$$
p_{[3]}^{-}=U_{v_{1}, v_{2}} V^{(1)}, \quad U_{v_{1}, v_{2}}=v_{1}^{N_{1}} v_{2}^{N_{2}}, \quad N_{1}=N_{B_{1}}+N_{\alpha_{12}}+N_{\alpha_{31}}, \quad N_{2}=N_{B_{2}}+N_{\alpha_{12}}+N_{\alpha_{23}},
$$

and using eqs. (F.8), we find that the vertex $V^{(1)}$ does not depend on the $v_{1}, v_{2}$, i.e., we get

$$
V^{(1)}=V^{(1)}\left(\beta_{a}, B_{a}, \alpha_{a a+1}\right) .
$$

Using (F.9), we find that, on vertex $V^{(1)}$ (F.10), operators $G_{a}, G_{\beta}$ (F.4)-(F.7) are realized as

$$
\begin{aligned}
G_{1}= & B_{3} \partial_{\alpha_{31}}-\left(B_{2}+\frac{\beta_{1}}{\beta_{2}} g_{2}\left(N_{2}+1\right)\right) \partial_{\alpha_{12}}+g_{1} \\
& +\frac{g_{1}^{(1)}\left(N_{1}+2\right)}{2 N_{1}+d-2}\left(\frac{2 \beta_{2}}{\beta_{1}} B_{2} \partial_{B_{1}} \partial_{\alpha_{12}}+\frac{2 \beta_{3}}{\beta_{1}} B_{3} \partial_{B_{1}} \partial_{\alpha_{31}}+2 \alpha_{23} \partial_{\alpha_{12}} \partial_{\alpha_{31}}\right), \\
G_{2}= & \left(B_{1}-\frac{\beta_{2}}{\beta_{1}} g_{1}\left(N_{1}+1\right)\right) \partial_{\alpha_{12}}-B_{3} \partial_{\alpha_{23}}+g_{2} \\
& +\frac{g_{2}^{(1)}\left(N_{2}+2\right)}{2 N_{2}+d-2}\left(\frac{2 \beta_{3}}{\beta_{2}} B_{3} \partial_{B_{2}} \partial_{\alpha_{23}}+\frac{2 \beta_{1}}{\beta_{2}} B_{1} \partial_{B_{2}} \partial_{\alpha_{12}}+2 \alpha_{31} \partial_{\alpha_{12}} \partial_{\alpha_{23}}\right),
\end{aligned}
$$




$$
\begin{aligned}
G_{3} & =\left(B_{2}-\frac{\beta_{3}}{\beta_{2}} g_{2}\left(N_{2}+1\right)\right) \partial_{\alpha_{23}}-\left(B_{1}+\frac{\beta_{3}}{\beta_{1}} g_{1}\left(N_{1}+1\right)\right) \partial_{\alpha_{31}} \\
G_{\beta} & =-\frac{1}{\beta} \mathbb{N}-\frac{1}{\beta_{1}^{2}} g_{1}\left(N_{1}+1\right) \partial_{B_{1}}-\frac{1}{\beta_{2}^{2}} g_{2}\left(N_{2}+1\right) \partial_{B_{2}} \\
g_{1} & \left.\equiv g_{v_{1}}\right|_{N_{v_{1}} \rightarrow N_{1}},\left.\quad g_{2} \equiv g_{v_{2}}\right|_{N_{v_{2}} \rightarrow N_{2}},\left.\quad g_{1}^{(1)} \equiv g_{v_{1}}\right|_{N_{v_{1}} \rightarrow N_{1}+1},\left.\quad g_{2}^{(1)} \equiv g_{v_{2}}\right|_{N_{v_{2}} \rightarrow N_{2}+1}
\end{aligned}
$$

Step 3. Realization on $\boldsymbol{V}^{(2)}$. We find it convenient to introduce a vertex $V^{(2)}$ by the relations

$$
\begin{aligned}
& V^{(1)}=U_{1,2} V^{(2)}, \quad U_{1,2}=\left(\frac{2^{N_{1}} \Gamma\left(N_{1}+\frac{d-2}{2}\right)}{\Gamma\left(N_{1}+1\right)} \frac{2^{N_{2}} \Gamma\left(N_{2}+\frac{d-2}{2}\right)}{\Gamma\left(N_{2}+1\right)}\right)^{1 / 2}, \\
& V^{(2)}=V^{(2)}\left(\beta_{a}, B_{a}, \alpha_{a a+1}\right)
\end{aligned}
$$

where, in (F.17), we show that the vertex $V^{(2)}$ depends on the same variables as vertex $V^{(1)}$ (F.10). On the vertex $V^{(2)}$, the realization of $G_{a}, G_{\beta}$ (F.11)-(F.14) takes more convenient form given by

$$
\begin{aligned}
G_{1}= & B_{3} \partial_{\alpha_{31}}-\left(B_{2}+\frac{\beta_{1}}{\beta_{2}} \kappa_{2}\right) \partial_{\alpha_{12}}+\frac{1}{\kappa_{1}} g_{1}^{2}\left(N_{1}+1\right) \\
& +\frac{\kappa_{1}}{2 N_{1}+d-2}\left(\frac{2 \beta_{2}}{\beta_{1}} B_{2} \partial_{B_{1}} \partial_{\alpha_{12}}+\frac{2 \beta_{3}}{\beta_{1}} B_{3} \partial_{B_{1}} \partial_{\alpha_{31}}+2 \alpha_{23} \partial_{\alpha_{12}} \partial_{\alpha_{31}}\right) \\
G_{2}= & \left(B_{1}-\frac{\beta_{2}}{\beta_{1}} \kappa_{1}\right) \partial_{\alpha_{12}}-B_{3} \partial_{\alpha_{23}}+\frac{1}{\kappa_{2}} g_{2}^{2}\left(N_{2}+1\right) \\
& +\frac{\kappa_{2}}{2 N_{2}+d-2}\left(\frac{2 \beta_{3}}{\beta_{2}} B_{3} \partial_{B_{2}} \partial_{\alpha_{23}}+\frac{2 \beta_{1}}{\beta_{2}} B_{1} \partial_{B_{2}} \partial_{\alpha_{12}}+2 \alpha_{31} \partial_{\alpha_{12}} \partial_{\alpha_{23}}\right) \\
G_{3}= & \left(B_{2}-\frac{\beta_{3}}{\beta_{2}} \kappa_{2}\right) \partial_{\alpha_{23}}-\left(B_{1}+\frac{\beta_{3}}{\beta_{1}} \kappa_{1}\right) \partial_{\alpha_{31}} \\
G_{\beta}= & -\frac{1}{\beta} \mathbb{N}-\frac{1}{\beta_{1}^{2}} \kappa_{1} \partial_{B_{1}}-\frac{1}{\beta_{2}^{2}} \kappa_{2} \partial_{B_{2}} .
\end{aligned}
$$

Namely, as compared with operators (F.11)-(F.14), operators (F.18)-(F.21) do not involve square roots of the operators $N_{1}, N_{2}$ (F.9). Note, it is the quantities $g_{a}, g_{a}^{(1)}, a=1,2$ (F.15) that enter square roots of the $N_{1}, N_{2}$.

Step 4. Realization on $\boldsymbol{V}_{\boldsymbol{Z} \boldsymbol{\beta}}^{(\mathbf{2})}$. We study dependence of $V^{(2)}$ (F.17) on the $\alpha_{23}$ and $\alpha_{31}$. Namely, we note that solution to equation $G_{3} V^{(2)}=0$ with $V^{(2)}, G_{3}$ as in (F.17), (F.20), is given by

$$
V^{(2)}=V_{Z \beta}^{(2)}\left(\beta_{a}, B_{a}, \alpha_{12}, Z\right), \quad Z=\left(B_{1}+\frac{\beta_{3}}{\beta_{1}} \kappa_{1}\right) \alpha_{23}+\left(B_{2}-\frac{\beta_{3}}{\beta_{2}} \kappa_{2}\right) \alpha_{31}
$$


Using (F.22), we find that, on vertex $V_{Z \beta}^{(2)}$ (F.22), operators $G_{1}, G_{2}$ (F.18), (F.19) are realized as

$$
\begin{aligned}
G_{1}= & B_{3}\left(B_{2}-\frac{\beta_{3}}{\beta_{2}} \kappa_{2}\right) \partial_{Z}-\left(B_{2}+\frac{\beta_{1}}{\beta_{2}} \kappa_{2}\right) \partial_{\alpha_{12}} \\
& +\frac{\kappa_{1}}{2 N_{1 Z}+d-2}\left(1+\frac{2 \beta_{2}}{\beta_{1}} B_{2} \partial_{B_{1}} \partial_{\alpha_{12}}+\frac{2 \beta_{3}}{\beta_{1}} B_{3}\left(B_{2}-\frac{\beta_{3}}{\beta_{2}} \kappa_{2}\right) \partial_{B_{1}} \partial_{Z}\right) \\
G_{2}= & \left(B_{1}-\frac{\beta_{2}}{\beta_{1}} \kappa_{1}\right) \partial_{\alpha_{12}}-B_{3}\left(B_{1}+\frac{\beta_{3}}{\beta_{1}} \kappa_{1}\right) \partial_{Z} \\
& +\frac{\kappa_{2}}{2 N_{2 Z}+d-2}\left(1+\frac{2 \beta_{3}}{\beta_{2}} B_{3}\left(B_{1}+\frac{\beta_{3}}{\beta_{1}} \kappa_{1}\right) \partial_{B_{2}} \partial_{Z}+\frac{2 \beta_{1}}{\beta_{2}} B_{1} \partial_{B_{2}} \partial_{\alpha_{12}}\right) \\
N_{1 Z}= & N_{B_{1}}+N_{\alpha_{12}}+N_{Z}, \quad N_{2 Z}=N_{B_{2}}+N_{\alpha_{12}}+N_{Z}
\end{aligned}
$$

Step 5. Analysis of equations for $\boldsymbol{V}_{\boldsymbol{Z} \boldsymbol{\beta}}^{(2)}$. We now analyse equations for vertex $V_{Z \beta}^{(2)}(\mathrm{F} .22)$,

$$
G_{1} V_{Z \beta}^{(2)}=0, \quad G_{2} V_{Z \beta}^{(2)}=0, \quad V_{Z \beta}^{(2)}=V_{Z \beta}^{(2)}\left(\beta_{a}, B_{a}, \alpha_{12}, Z\right)
$$

where $G_{1}, G_{2}$ are given in (F.23)-(F.25). As the vertex $V_{Z \beta}^{(2)}$ is considered to be expandable in the $B_{3}$ we use the following power series expansion:

$$
V_{Z \beta}^{(2)}=\sum_{n=0}^{\infty} V_{n}, \quad N_{B_{3}} V_{n}=n V_{n}, \quad V_{n}=V_{n}\left(\beta_{a}, B_{a}, \alpha_{12}, Z\right),
$$

where $V_{n}$ is a degree- $n$ monomial in $B_{3}$. Equations (F.26) then imply

$$
\begin{aligned}
G_{1} V_{0} & =0, \quad G_{2} V_{0}=0, \\
G_{1} & =-\left(B_{2}+\frac{\beta_{1}}{\beta_{2}} \kappa_{2}\right) \partial_{\alpha_{12}}+\frac{\kappa_{1}}{2 N_{1 Z}+d-2}\left(1+\frac{2 \beta_{2}}{\beta_{1}} B_{2} \partial_{B_{1}} \partial_{\alpha_{12}}\right), \\
G_{2} & =\left(B_{1}-\frac{\beta_{2}}{\beta_{1}} \kappa_{1}\right) \partial_{\alpha_{12}}+\frac{\kappa_{2}}{2 N_{2 Z}+d-2}\left(1+\frac{2 \beta_{1}}{\beta_{2}} B_{1} \partial_{B_{2}} \partial_{\alpha_{12}}\right),
\end{aligned}
$$

where $N_{1 Z}, N_{2 Z}$ are given in (F.25). As $V_{0}$ is considered to be expandable in the $B_{1}, B_{2}$, we use the following power series expansion in the $B_{1}, B_{2}$ :

$$
V_{0}=\sum_{n=0}^{\infty} \bar{V}_{n}, \quad\left(N_{B_{1}}+N_{B_{2}}\right) \bar{V}_{n}=n \bar{V}_{n}, \quad \bar{V}_{n}=\bar{V}_{n}\left(\beta_{a}, B_{1}, B_{2}, \alpha_{12}, Z\right) .
$$

From (F.28)-(F.31), we get the following equations for the vertex $\bar{V}_{0}$ :

$$
\begin{aligned}
& \left(-\left(2 N_{1 Z}+d-2\right) \partial_{\alpha_{12}}+\frac{\kappa_{1} \beta_{2}}{\kappa_{2} \beta_{1}}\right) \bar{V}_{0}=0, \\
& \left(-\left(2 N_{2 Z}+d-2\right) \partial_{\alpha_{12}}+\frac{\kappa_{2} \beta_{1}}{\kappa_{1} \beta_{2}}\right) \bar{V}_{0}=0,
\end{aligned}
$$

where $N_{1 Z}, N_{2 Z}$ are given in (F.25). Subtracting (F.33) from (F.32), we get the equation

$$
\left(\frac{\kappa_{1} \beta_{2}}{\kappa_{2} \beta_{1}}-\frac{\kappa_{2} \beta_{1}}{\kappa_{1} \beta_{2}}\right) \bar{V}_{0}=0
$$


which implies

$$
\bar{V}_{0}=0 .
$$

Using (F.35) in (F.28)-(F.31), we get the following equations for the vertex $\bar{V}_{1}$ :

$$
\begin{aligned}
& \left(-\left(2 N_{1 Z}+d-2\right) \partial_{\alpha_{12}}+\frac{2 \kappa_{1} \beta_{2}^{2}}{\kappa_{2} \beta_{1}^{2}} B_{2} \partial_{B_{1}} \partial_{\alpha_{12}}+\frac{\kappa_{1} \beta_{2}}{\kappa_{2} \beta_{1}}\right) \bar{V}_{1}=0, \\
& \left(-\left(2 N_{2 Z}+d-2\right) \partial_{\alpha_{12}}+\frac{2 \kappa_{2} \beta_{1}^{2}}{\kappa_{1} \beta_{2}^{2}} B_{1} \partial_{B_{2}} \partial_{\alpha_{12}}+\frac{\kappa_{2} \beta_{1}}{\kappa_{1} \beta_{2}}\right) \bar{V}_{1}=0
\end{aligned}
$$

where $N_{1 Z}, N_{2 Z}$ are given in (F.25). Subtracting (F.37) from (F.36), we get the equation

$$
\left(\left(-2 N_{B_{1}}+2 N_{B_{2}}\right) \partial_{\alpha_{12}}+\frac{2 \kappa_{1} \beta_{2}^{2}}{\kappa_{2} \beta_{1}^{2}} B_{2} \partial_{B_{1}} \partial_{\alpha_{12}}-\frac{2 \kappa_{2} \beta_{1}^{2}}{\kappa_{1} \beta_{2}^{2}} B_{1} \partial_{B_{2}} \partial_{\alpha_{12}}+\frac{\kappa_{1} \beta_{2}}{\kappa_{2} \beta_{1}}-\frac{\kappa_{2} \beta_{1}}{\kappa_{1} \beta_{2}}\right) \bar{V}_{1}=0
$$

Now, in place of the $B_{1}, B_{2}$, we introduce new variables $B_{ \pm}$,

$$
B_{ \pm}=\frac{\beta_{1}^{2}}{\kappa_{1}} B_{1} \pm \frac{\beta_{2}^{2}}{\kappa_{2}} B_{2}
$$

and note that, in terms of the $B_{ \pm}$, equation (F.38) takes the form

$$
\left(-4 B_{-} \partial_{B_{+}} \partial_{\alpha_{12}}+\frac{\kappa_{1} \beta_{2}}{\kappa_{2} \beta_{1}}-\frac{\kappa_{2} \beta_{1}}{\kappa_{1} \beta_{2}}\right) \bar{V}_{1}=0
$$

As the vertex $\bar{V}_{1}$ is a degree-1 homogeneous polynomial in the $B_{+}, B_{-}$(F.31), we use the expansion

$$
\bar{V}_{1}=B_{+} \bar{V}_{1+}+B_{-} \bar{V}_{1-}
$$

and verify that eq. (F.40) amounts to the equations

$$
-4 \partial_{\alpha_{12}} \bar{V}_{1+}+\left(\frac{\kappa_{1} \beta_{2}}{\kappa_{2} \beta_{1}}-\frac{\kappa_{2} \beta_{1}}{\kappa_{1} \beta_{2}}\right) \bar{V}_{1-}=0, \quad\left(\frac{\kappa_{1} \beta_{2}}{\kappa_{2} \beta_{1}}-\frac{\kappa_{2} \beta_{1}}{\kappa_{1} \beta_{2}}\right) \bar{V}_{1+}=0 .
$$

The 2nd equation in (F.42) implies $\bar{V}_{1+}=0$. Setting then $\bar{V}_{1+}=0$ in the 1 st equation in (F.42), we find $\bar{V}_{1-}=0$, i.e., vertex $\bar{V}_{1}$ (F.41) is trivial,

$$
\bar{V}_{1}=0 \text {. }
$$

Thus we have demonstrated that eqs. (F.28) and relations in (F.29)-(F.31) lead to $\bar{V}_{0}=0$ and $\bar{V}_{1}=0$. We now use the induction method. Suppose eqs. (F.28)-(F.31) lead to trivial $\bar{V}_{k}=0$ for $k=0,1, \ldots, n-1$. Then we are going to prove that $\bar{V}_{n}=0$. To this end we note that if $\bar{V}_{k}=0$, for $k=0,1, \ldots n-1$ then eqs. (F.28) and relations in (F.29)-(F.31) lead to the equation for $\bar{V}_{n}$ which is obtained by replacement $\bar{V}_{1} \rightarrow \bar{V}_{n}$ in (F.36), (F.37). Therefore repeating analysis we used above for the vertex $\bar{V}_{1}$, we obtain the following equation for the vertex $\bar{V}_{n}$ :

$$
\left(-4 B_{-} \partial_{B_{+}} \partial_{\alpha_{12}}+\frac{\kappa_{1} \beta_{2}}{\kappa_{2} \beta_{1}}-\frac{\kappa_{2} \beta_{1}}{\kappa_{1} \beta_{2}}\right) \bar{V}_{n}=0
$$


By definition (F.31), the vertex $\bar{V}_{n}$ is a degree- $n$ homogeneous polynomial in variables $B_{ \pm}$(F.39). Therefore, we can present the vertex $\bar{V}_{n}$ as

$$
\bar{V}_{n}=\sum_{k=0}^{n} B_{+}^{k} B_{-}^{n-k} \bar{V}_{n, k},
$$

where $\bar{V}_{n, k}$ are independent of the $B_{ \pm}$. Plugging (F.45) into (F.44), we verify that $\bar{V}_{n, k}=0$ for all $k=0,1, \ldots n$, i.e., $\bar{V}_{n}=0$. Using the induction method, we conclude then that $\bar{V}_{n}=0$ for all $n=0,1, \ldots \infty$. In other words, $V_{0}=0$ (F.31). Using the relation $V_{0}=0$ and applying the induction method to the vertices $V_{n}$ in (F.27) we find therefore that $V_{n}=0$ for all $n=0,1, \ldots, \infty$. Thus we conclude that $V_{Z \beta}^{(2)}=0$ (F.27). This implies that vertex (F.2) is trivial, $p_{[3]}^{-}=0$.

Open Access. This article is distributed under the terms of the Creative Commons Attribution License (CC-BY 4.0), which permits any use, distribution and reproduction in any medium, provided the original author(s) and source are credited.

\section{References}

[1] X. Bekaert and J. Mourad, The continuous spin limit of higher spin field equations, JHEP 01 (2006) 115 [hep-th/0509092] [INSPIRE].

[2] X. Bekaert and N. Boulanger, The unitary representations of the Poincaré group in any spacetime dimension, hep-th/0611263 [INSPIRE].

[3] P. Schuster and N. Toro, Continuous-spin particle field theory with helicity correspondence, Phys. Rev. D 91 (2015) 025023 [arXiv: 1404.0675] [INSPIRE].

[4] V.O. Rivelles, Gauge Theory Formulations for Continuous and Higher Spin Fields, Phys. Rev. D 91 (2015) 125035 [arXiv:1408.3576] [INSPIRE].

[5] X. Bekaert, M. Najafizadeh and M.R. Setare, A gauge field theory of fermionic Continuous-Spin Particles, Phys. Lett. B 760 (2016) 320 [arXiv:1506.00973] [InSPIRE].

[6] R.R. Metsaev, Continuous spin gauge field in (A)dS space, Phys. Lett. B 767 (2017) 458 [arXiv: 1610.00657] [INSPIRE].

[7] R.R. Metsaev, Fermionic continuous spin gauge field in (A)dS space, Phys. Lett. B 773 (2017) 135 [arXiv: 1703.05780] [INSPIRE].

[8] Yu. M. Zinoviev, Infinite spin fields in D=3 and beyond, Universe 3 (2017) 63 [arXiv: 1707.08832] [INSPIRE].

[9] X. Bekaert and E.D. Skvortsov, Elementary particles with continuous spin, Int. J. Mod. Phys. A 32 (2017) 1730019 [arXiv:1708.01030] [INSPIRE].

[10] M. Najafizadeh, Modified Wigner equations and continuous spin gauge field, arXiv: 1708.00827 [INSPIRE].

[11] L. Brink, A.M. Khan, P. Ramond and X.-z. Xiong, Continuous spin representations of the Poincaré and superPoincaré groups, J. Math. Phys. 43 (2002) 6279 [hep-th/0205145] [INSPIRE].

[12] G.K. Savvidy, Tensionless strings: Physical Fock space and higher spin fields, Int. J. Mod. Phys. A 19 (2004) 3171 [hep-th/0310085] [INSPIRE]. 
[13] J. Mourad, Continuous spin particles from a string theory, hep-th/0504118 [INSPIRE].

[14] M.A. Vasiliev, Consistent equation for interacting gauge fields of all spins in (3+1)-dimensions, Phys. Lett. B 243 (1990) 378 [inSPIRE].

[15] M.A. Vasiliev, Nonlinear equations for symmetric massless higher spin fields in (A)dS(d), Phys. Lett. B 567 (2003) 139 [hep-th/0304049] [INSPIRE].

[16] P.A.M. Dirac, Forms of Relativistic Dynamics, Rev. Mod. Phys. 21 (1949) 392 [inSPIRE].

[17] M.B. Green, J.H. Schwarz and L. Brink, Superfield Theory of Type II Superstrings, Nucl. Phys. B 219 (1983) 437 [inSPIRE].

[18] M.B. Green and J.H. Schwarz, Superstring Interactions, Nucl. Phys. B 218 (1983) 43 [INSPIRE].

[19] M.B. Green and J.H. Schwarz, Superstring Field Theory, Nucl. Phys. B 243 (1984) 475 [INSPIRE].

[20] R.R. Metsaev, Eleven dimensional supergravity in light cone gauge, Phys. Rev. D 71 (2005) 085017 [hep-th/0410239] [INSPIRE].

[21] S. Ananth, L. Brink and P. Ramond, Eleven-dimensional supergravity in light-cone superspace, JHEP 05 (2005) 003 [hep-th/0501079] [INSPIRE].

[22] S. Ananth, Gravity as the square of Yang-Mills: Implications for $N=8$ Supergravity, Fortsch. Phys. 57 (2009) 857 [arXiv:0902.3128] [InSPIRE].

[23] S. Ananth, L. Brink and M. Mali, Yang-Mills theories and quadratic forms, JHEP 08 (2015) 153 [arXiv: 1507.01068] [INSPIRE].

[24] S.J. Brodsky, G.F. de Téramond and H.G. Dosch, QCD on the Light-Front - A Systematic Approach to Hadron Physics, Few Body Syst. 55 (2014) 407 [arXiv: 1310.8648] [INSPIRE].

[25] S.J. Brodsky, G.F. de Teramond, H.G. Dosch and J. Erlich, Light-Front Holographic QCD and Emerging Confinement, Phys. Rept. 584 (2015) 1 [arXiv:1407.8131] [INSPIRE].

[26] S.J. Brodsky, G.F. de Téramond, H.G. Dosch and C. Lorcé, Meson/Baryon/Tetraquark Supersymmetry from Superconformal Algebra and Light-Front Holography, Int. J. Mod. Phys. A 31 (2016) 1630029 [arXiv:1606. 04638] [INSPIRE].

[27] A.K.H. Bengtsson, I. Bengtsson and L. Brink, Cubic Interaction Terms for Arbitrary Spin, Nucl. Phys. B 227 (1983) 31 [INSPIRE].

[28] A.K.H. Bengtsson, I. Bengtsson and L. Brink, Cubic Interaction Terms for Arbitrarily Extended Supermultiplets, Nucl. Phys. B 227 (1983) 41 [INSPIRE].

[29] A.K.H. Bengtsson, I. Bengtsson and N. Linden, Interacting Higher Spin Gauge Fields on the Light Front, Class. Quant. Grav. 4 (1987) 1333 [InSPIRE].

[30] R.R. Metsaev, Generating function for cubic interaction vertices of higher spin fields in any dimension, Mod. Phys. Lett. A 8 (1993) 2413 [InSPIRE].

[31] R.R. Metsaev, Cubic interaction vertices of massive and massless higher spin fields, Nucl. Phys. B 759 (2006) 147 [hep-th/0512342] [INSPIRE].

[32] R.R. Metsaev, Cubic interaction vertices for fermionic and bosonic arbitrary spin fields, Nucl. Phys. B 859 (2012) 13 [arXiv:0712.3526] [INSPIRE].

[33] W. Siegel, Introduction to string field theory, Adv. Ser. Math. Phys. 8 (1988) 1 [hep-th/0107094] [INSPIRE]. 
[34] R.R. Metsaev, Light cone form of field dynamics in Anti-de Sitter space-time and AdS/CFT correspondence, Nucl. Phys. B 563 (1999) 295 [hep-th/9906217] [INSPIRE].

[35] J.E. Paton and H.-M. Chan, Generalized veneziano model with isospin, Nucl. Phys. B 10 (1969) 516 [INSPIRE].

[36] R.R. Metsaev, $S$ matrix approach to massless higher spins theory. 2: The case of internal symmetry, Mod. Phys. Lett. A 6 (1991) 2411 [INSPIRE].

[37] A. Fotopoulos and M. Tsulaia, On the Tensionless Limit of String theory, Off-Shell Higher Spin Interaction Vertices and BCFW Recursion Relations, JHEP 11 (2010) 086 [arXiv: 1009.0727] [INSPIRE].

[38] R.R. Metsaev, BRST-BV approach to cubic interaction vertices for massive and massless higher-spin fields, Phys. Lett. B 720 (2013) 237 [arXiv:1205.3131] [INSPIRE].

[39] A. Sagnotti and M. Taronna, String Lessons for Higher-Spin Interactions, Nucl. Phys. B 842 (2011) 299 [arXiv:1006.5242] [INSPIRE].

[40] R. Manvelyan, K. Mkrtchyan and W. Rühl, General trilinear interaction for arbitrary even higher spin gauge fields, Nucl. Phys. B 836 (2010) 204 [arXiv: 1003.2877] [INSPIRE].

[41] R. Manvelyan, K. Mkrtchyan and W. Ruehl, A generating function for the cubic interactions of higher spin fields, Phys. Lett. B 696 (2011) 410 [arXiv:1009.1054] [INSPIRE].

[42] X. Bekaert, N. Boulanger and S. Cnockaert, Spin three gauge theory revisited, JHEP 01 (2006) 052 [hep-th/0508048] [INSPIRE].

[43] M. Henneaux, G. Lucena Gómez and R. Rahman, Higher-Spin Fermionic Gauge Fields and Their Electromagnetic Coupling, JHEP 08 (2012) 093 [arXiv: 1206.1048] [INSPIRE].

[44] M. Henneaux, G. Lucena Gómez and R. Rahman, Gravitational Interactions of Higher-Spin Fermions, JHEP 01 (2014) 087 [arXiv:1310.5152] [INSPIRE].

[45] F.W.J. Olver ed., NIST handbook of mathematical functions hardback and CD-ROM, Cambridge University Press, (2010).

[46] E.S. Fradkin and M.A. Vasiliev, On the Gravitational Interaction of Massless Higher Spin Fields, Phys. Lett. B 189 (1987) 89 [inSPIRE].

[47] M.A. Vasiliev, Cubic Vertices for Symmetric Higher-Spin Gauge Fields in $(A) d S_{d}$, Nucl. Phys. B 862 (2012) 341 [arXiv: 1108.5921] [InSPIRE].

[48] K. Alkalaev, FV-type action for AdS $S_{5}$ mixed-symmetry fields, JHEP 03 (2011) 031 [arXiv: 1011.6109] [INSPIRE].

[49] N. Boulanger, E.D. Skvortsov and Yu. M. Zinoviev, Gravitational cubic interactions for a simple mixed-symmetry gauge field in AdS and flat backgrounds, J. Phys. A 44 (2011) 415403 [arXiv: 1107.1872 ] [INSPIRE].

[50] N. Boulanger and E.D. Skvortsov, Higher-spin algebras and cubic interactions for simple mixed-symmetry fields in AdS spacetime, JHEP 09 (2011) 063 [arXiv:1107.5028] [INSPIRE].

[51] E. Joung and M. Taronna, Cubic interactions of massless higher spins in (A)dS: metric-like approach, Nucl. Phys. B 861 (2012) 145 [arXiv:1110.5918] [InSPIRE].

[52] E. Joung, L. Lopez and M. Taronna, Generating functions of (partially-)massless higher-spin cubic interactions, JHEP 01 (2013) 168 [arXiv:1211.5912] [INSPIRE]. 
[53] E. Joung, L. Lopez and M. Taronna, Solving the Noether procedure for cubic interactions of higher spins in (A)dS, J. Phys. A 46 (2013) 214020 [arXiv:1207.5520] [INSPIRE].

[54] C. Sleight and M. Taronna, Higher Spin Interactions from Conformal Field Theory: The Complete Cubic Couplings, Phys. Rev. Lett. 116 (2016) 181602 [arXiv:1603.00022] [INSPIRE].

[55] C. Sleight and M. Taronna, Feynman rules for higher-spin gauge fields on $A d S_{d+1}$, arXiv: 1708.08668 [INSPIRE].

[56] D. Francia, G.L. Monaco and K. Mkrtchyan, Cubic interactions of Maxwell-like higher spins, JHEP 04 (2017) 068 [arXiv:1611.00292] [INSPIRE].

[57] D.S. Ponomarev and M.A. Vasiliev, Frame-Like Action and Unfolded Formulation for Massive Higher-Spin Fields, Nucl. Phys. B 839 (2010) 466 [arXiv: 1001.0062] [INSPIRE].

[58] O.A. Gelfond and M.A. Vasiliev, Current Interactions from the One-Form Sector of Nonlinear Higher-Spin Equations, arXiv: 1706.03718 [INSPIRE].

[59] N. Misuna, On current contribution to Fronsdal equations, arXiv:1706.04605 [INSPIRE].

[60] R. Bonezzi, Induced Action for Conformal Higher Spins from Worldline Path Integrals, Universe 3 (2017) 64 [arXiv: 1709. 00850] [INSPIRE].

[61] T. Adamo, P. Hähnel and T. McLoughlin, Conformal higher spin scattering amplitudes from twistor space, JHEP 04 (2017) 021 [arXiv: 1611.06200] [INSPIRE].

[62] P. Haehnel and T. McLoughlin, Conformal Higher Spin Theory and Twistor Space Actions, J. Phys. A 50 (2017) 485401 [arXiv: 1604.08209] [INSPIRE].

[63] D.V. Uvarov, Ambitwistors, oscillators and massless fields on AdS $S_{5}$, Phys. Lett. B 762 (2016) 415 [arXiv: 1607.05233] [INSPIRE].

[64] D.V. Uvarov, Spinning particle and null-string on AdS $S_{d}$ : projective-space approach, arXiv:1707.05761 [INSPIRE].

[65] M. Grigoriev and A.A. Tseytlin, On conformal higher spins in curved background, J. Phys. A 50 (2017) 125401 [arXiv: 1609.09381] [INSPIRE].

[66] R.R. Metsaev, Massless arbitrary spin fields in AdS $S_{5}$, Phys. Lett. B 531 (2002) 152 [hep-th/0201226] [INSPIRE].

[67] R.R. Metsaev, Massive totally symmetric fields in AdS(d), Phys. Lett. B 590 (2004) 95 [hep-th/0312297] [INSPIRE].

[68] R.R. Metsaev, Extended Hamiltonian Action for Arbitrary Spin Fields in Flat And AdS Spaces, J. Phys. A 46 (2013) 214021 [arXiv:1112.0976] [INSPIRE].

[69] M.A. Vasiliev, Linearized Curvatures for Auxiliary Fields in the de Sitter Space, Nucl. Phys. B 307 (1988) 319 [INSPIRE].

[70] A. Campoleoni, M. Henneaux, S. Hörtner and A. Leonard, Higher-spin charges in Hamiltonian form. I. Bose fields, JHEP 10 (2016) 146 [arXiv:1608.04663] [inSPIRE].

[71] A. Leonard, Aspects of higher spin Hamiltonian dynamics: Conformal geometry, duality and charges, arXiv:1709.00719 [INSPIRE].

[72] I.L. Buchbinder and K. Koutrolikos, BRST Analysis of the Supersymmetric Higher Spin Field Models, JHEP 12 (2015) 106 [arXiv:1510.06569] [inSPIRE]. 
[73] I.L. Buchbinder, S.J. Gates and K. Koutrolikos, Higher Spin Superfield interactions with the Chiral Supermultiplet: Conserved Supercurrents and Cubic Vertices, arXiv:1708.06262 [INSPIRE].

[74] S.M. Kuzenko and M. Tsulaia, Off-shell massive $N=1$ supermultiplets in three dimensions, Nucl. Phys. B 914 (2017) 160 [arXiv:1609.06910] [InSPIRE].

[75] A.K.H. Bengtsson, BRST Theory for Continuous Spin, JHEP 10 (2013) 108 [arXiv: 1303.3799] [INSPIRE].

[76] D. Francia and A. Sagnotti, Higher-spin geometry and string theory, J. Phys. Conf. Ser. 33 (2006) 57 [hep-th/0601199] [INSPIRE].

[77] A. Sagnotti and M. Tsulaia, On higher spins and the tensionless limit of string theory, Nucl. Phys. B 682 (2004) 83 [hep-th/0311257] [INSPIRE].

[78] D. Francia, String theory triplets and higher-spin curvatures, Phys. Lett. B 690 (2010) 90 [arXiv: 1001.5003 ] [INSPIRE].

[79] A. Campoleoni, D. Francia, J. Mourad and A. Sagnotti, Unconstrained Higher Spins of Mixed Symmetry. I. Bose Fields, Nucl. Phys. B 815 (2009) 289 [arXiv:0810.4350] [INSPIRE].

[80] R.R. Metsaev, BRST-BV approach to massless fields adapted to AdS/CFT correspondence, Theor. Math. Phys. 187 (2016) 730 [arXiv:1508. 07928] [InSPIRE].

[81] R.R. Metsaev, The BRST-BV approach to conformal fields, J. Phys. A 49 (2016) 175401 [arXiv: 1511.01836] [INSPIRE].

[82] R.R. Metsaev, BRST invariant effective action of shadow fields, conformal fields and AdS/CFT, Theor. Math. Phys. 181 (2014) 1548 [arXiv:1407.2601] [INSPIRE].

[83] R.R. Metsaev, Arbitrary spin conformal fields in (A)dS, Nucl. Phys. B 885 (2014) 734 [arXiv:1404.3712] [INSPIRE].

[84] N. Boulanger, C. Iazeolla and P. Sundell, Unfolding Mixed-Symmetry Fields in AdS and the BMV Conjecture: I. General Formalism, JHEP 07 (2009) 013 [arXiv:0812.3615] [INSPIRE].

[85] N. Boulanger, C. Iazeolla and P. Sundell, Unfolding Mixed-Symmetry Fields in AdS and the BMV Conjecture. II. Oscillator Realization, JHEP 07 (2009) 014 [arXiv:0812.4438] [INSPIRE].

[86] E.D. Skvortsov, Gauge fields in (A)dS(d) and Connections of its symmetry algebra, J. Phys. A 42 (2009) 385401 [arXiv:0904.2919] [INSPIRE].

[87] E.D. Skvortsov, Gauge fields in (A)dS(d) within the unfolded approach: algebraic aspects, JHEP 01 (2010) 106 [arXiv:0910.3334] [INSPIRE].

[88] K.B. Alkalaev and M. Grigoriev, Unified BRST description of AdS gauge fields, Nucl. Phys. B 835 (2010) 197 [arXiv:0910.2690] [InSPIRE].

[89] K. Alkalaev and M. Grigoriev, Unified BRST approach to (partially) massless and massive AdS fields of arbitrary symmetry type, Nucl. Phys. B 853 (2011) 663 [arXiv:1105.6111] [INSPIRE].

[90] I.L. Buchbinder and A. Reshetnyak, General Lagrangian Formulation for Higher Spin Fields with Arbitrary Index Symmetry. I. Bosonic fields, Nucl. Phys. B 862 (2012) 270 [arXiv:1110.5044] [INSPIRE]. 
[91] C. Burdik and A. Reshetnyak, On representations of Higher Spin symmetry algebras for mixed-symmetry HS fields on AdS-spaces. Lagrangian formulation, J. Phys. Conf. Ser. 343 (2012) 012102 [arXiv:1111.5516] [INSPIRE].

[92] R.R. Metsaev, Mixed symmetry massive fields in AdS $S_{5}$, Class. Quant. Grav. 22 (2005) 2777 [hep-th/0412311] [INSPIRE].

[93] R.R. Metsaev, Light-cone AdS/CFT-adapted approach to AdS fields/currents, shadows and conformal fields, JHEP 10 (2015) 110 [arXiv:1507.06584] [INSPIRE].

[94] R.R. Metsaev, Mixed-symmetry fields in AdS $S_{5}$, conformal fields and AdS/CFT, JHEP 01 (2015) 077 [arXiv: 1410.7314] [INSPIRE].

[95] F. Bastianelli, R. Bonezzi, O. Corradini and E. Latini, Massive and massless higher spinning particles in odd dimensions, JHEP 09 (2014) 158 [arXiv:1407.4950] [INSPIRE].

[96] R.R. Metsaev, Poincaré invariant dynamics of massless higher spins: Fourth order analysis on mass shell, Mod. Phys. Lett. A 6 (1991) 359 [inSPIRE].

[97] D. Ponomarev and E.D. Skvortsov, Light-Front Higher-Spin Theories in Flat Space, J. Phys. A 50 (2017) 095401 [arXiv: 1609.04655] [InSPIRE].

[98] M. Taronna, On the Non-Local Obstruction to Interacting Higher Spins in Flat Space, JHEP 05 (2017) 026 [arXiv:1701.05772] [INSPIRE].

[99] R. Roiban and A.A. Tseytlin, On four-point interactions in massless higher spin theory in flat space, JHEP 04 (2017) 139 [arXiv: 1701.05773] [INSPIRE].

[100] C. Sleight, Interactions in Higher-Spin Gravity: a Holographic Perspective, J. Phys. A 50 (2017) 383001 [arXiv: 1610.01318] [INSPIRE].

[101] P. Dempster and M. Tsulaia, On the Structure of Quartic Vertices for Massless Higher Spin Fields on Minkowski Background, Nucl. Phys. B 865 (2012) 353 [arXiv:1203.5597] [INSPIRE]. 